Supplement of Hydrol. Earth Syst. Sci., 21, 2863-2879, 2017

https://doi.org/10.5194/hess-21-2863-2017-supplement

(C) Author(s) 2017. This work is distributed under

the Creative Commons Attribution 3.0 License.

(c) (1)

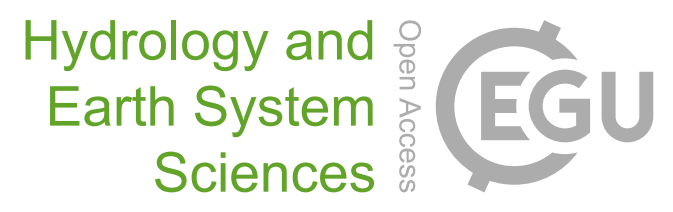

Supplement of

\title{
Understanding hydrologic variability across Europe through catchment classification
}

\author{
Anna Kuentz et al. \\ Correspondence to: Anna Kuentz (anna.kuentz@smhi.se)
}

The copyright of individual parts of the supplement might differ from the CC BY 3.0 License. 
Table of Content:

A. CORRELATION ANALYSIS

B. CART TREE NODES DESCRIPTION

C. MATERIAL FOR THE ANALYSIS OF THE CART CLASSIFICATION (BASE MATERIAL FOR TABLE 3) $\mathbf{5}$

C.1 Flow Signatures 5

C.1.1 DISTRIBUTION (BOXPLOTS) OF FLOW SIGNATURES IN THE DIFFERENT CLASSES

C.1.2 MATRIX OF MEDIAN FLOW SIGNATURES FOR EACH CLASS COMPARED TO THE WHOLE SET OF STREAM GAUGES 8

C.2 CATCHMENT DESCRIPTORS 9

C.2.1 DISTRIBUTION (BOXPLOTS) OF CATCHMENT DESCRIPTORS IN THE DIFFERENT CLASSES 9

C.2.2 MATRIX OF MEDIAN CATCHMENT DESCRIPTORS FOR EACH CLASS COMPARED TO THE WHOLE SET OF STREAM

GAUGES / CATCHMENTS 16

C.3 LARGER MAP OF THE CLASSIFICATION AND DETAILED MAPS FOR CLASSES CORRESPONDING TO MORE THAN ONE NODE IN THE CART TREE $\quad 17$

D. DISTRIBUTION (BOXPLOTS) OF CATCHMENT DESCRIPTORS AND FLOW SIGNATURES IN THE DIFFERENT CLASSES OF FS AND CD CLASSIFICATIONS (PLOTS ARE COMMENTED IN SECTION 3.2) 19

D.1 CLASSIFICATION BASED ON FLOW SIGNATURES (FS CLASSIFICATION) 19

D.1.1 BOXPLOTS OF FLOW SIGNATURES

D.1.2 BOXPLOTS OF CATCHMENT DESCRIPTORS 22

D.2 CLASSIFICATION BASED ON CATCHMENT DESCRIPTORS 29

D.2.1 BOXPLOTS OF FLOW SIGNATURES

D.2.2 BOXPLOTS OF CATCHMENT DESCRIPTORS

E. LINEAR REGRESSION COEFFICIENTS

E.1 LINEAR REgRESSIONS CALIBRATED OVER THE WHOLE DOMAIN $\quad 39$

E.2 LINEAR REGRESSIONS CALIBRATED INSIDE EACH CLASSIFICATION GROUP 40

E.2.1 CART CLASSIFICATION

$\begin{array}{ll}\text { E.2.2 CLASSIFICATION BASED ON FLOW SIGNATURES } & 50\end{array}$

E.2.3 CLASSIFICATION BASED ON CATCHMENT DESCRIPTORS 60 


\section{A. Correlation analysis}

Significant correlations (based on a t distribution with a threshold of 0.05) were found for 400 (out of 786) correlations between flow signatures and catchment descriptors (Fig. A); for instance positive correlation between mean slope and specific flow or low flows, and negative correlation between agricultural area and runoff ratio, and between aridity index and specific flow, 5th and 95th percentiles and runoff ratio. Overall, we found relationships to be consistent with our a priori knowledge (e.g. Donnelly et al, 2016).

As shown in Fig. A, there were no big differences between the three types of correlations compared. As expected, more significant correlations appear when using Spearman correlation than Pearson correlation, but the coloring shows that the differences are not very large. Pearson and distance correlation matrices also exhibit similar patterns (putting aside that distance correlation cannot be negative). More significant correlations were found when using distance correlation compared to Pearson, but still less than when using the Spearman correlation metric. One exception appears however, which is the percentage of "Cenozoic-Mesozoic igneous" (CzMzi) geological class. This percentage appears to be highly correlated with some of the flow signatures including the skewness of daily flow, the high flow discharge and the mean 30-days maximum. These high correlations (around 0.8) are noticeable in the Pearson (Fig Aa) and distance matrices (Fig. Ac), but absent from the Spearman matrix (Fig Ab). This high correlation led to further examination of the scatterplot between the two variables. It appeared that only a few catchments contain a significant percentage of this geological class and the scatterplot shows that the high correlation is only due to a few points with high values for both "CzMzi" and the concerned flow signature. These high correlations were thus ignored in the following analysis. In addition, combined analysis of the Pearson correlation matrix and the scatterplots showed that a number of catchment descriptors did not have any significant relationship with any of the flow signatures (or only very low correlations - below 0.15 ). To simplify the process by reducing the number of variables, this led us to remove the following geological catchment descriptors for the rest of the analyses: CzMzi, Czv, i, m, Mzi, Mzm, MzPz, MzPzm, Mzv, pCmv, PzpCm, Pzv and karst. The low correlation of these variables could be due to small areal representativeness in the geographical domain, poor data quality or small influence of subsurface geology on surface hydrology. 


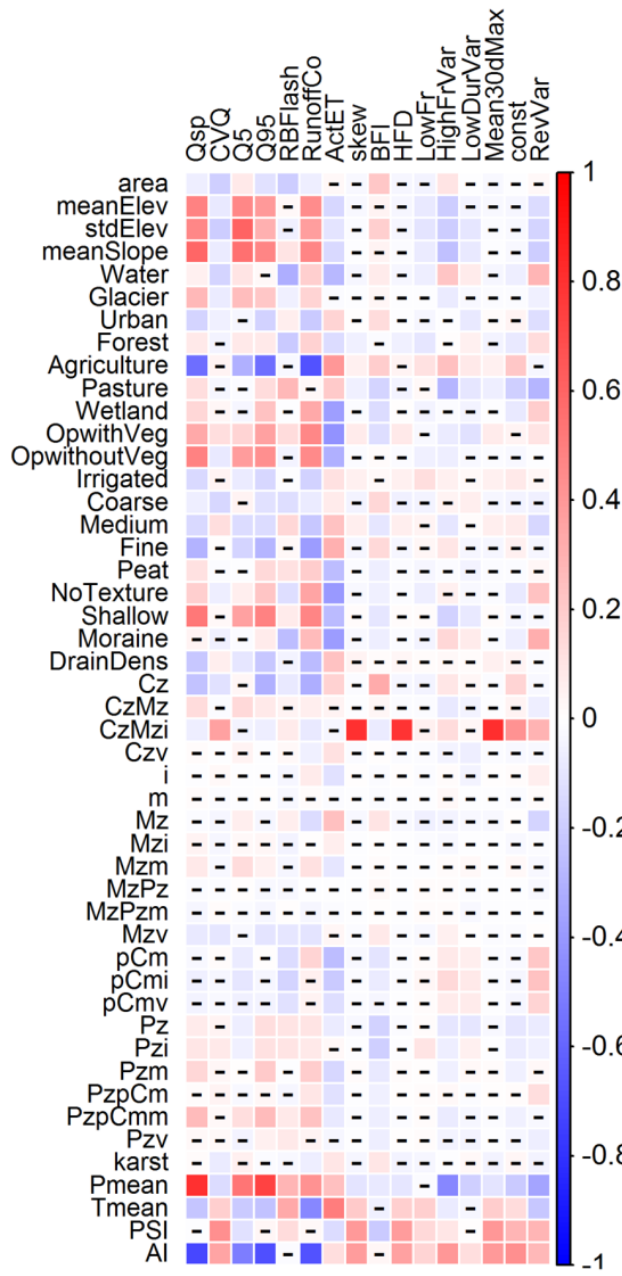

a. Pearson correlation

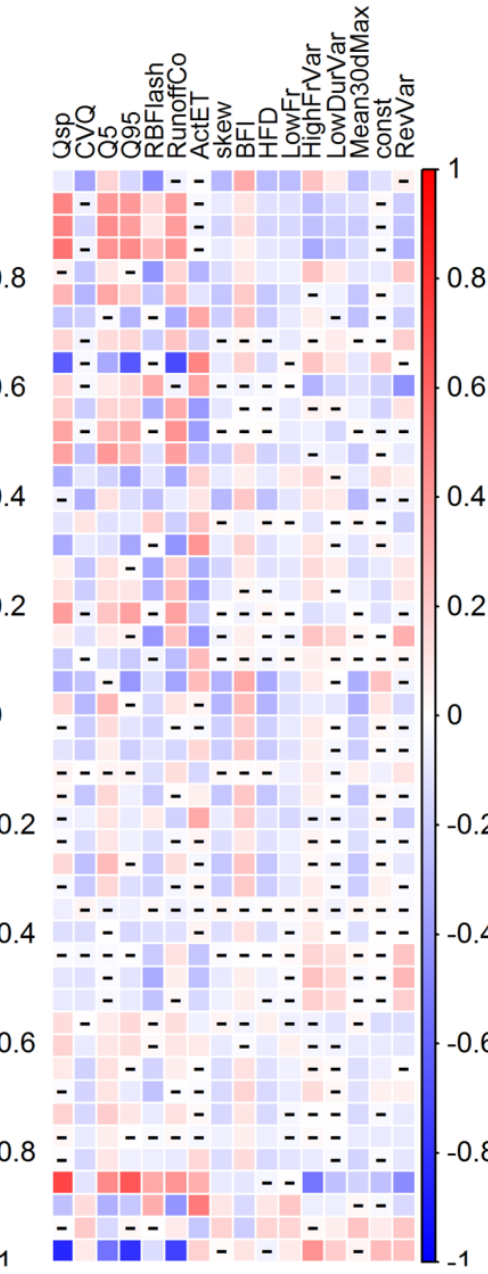

b. Spearman correlation

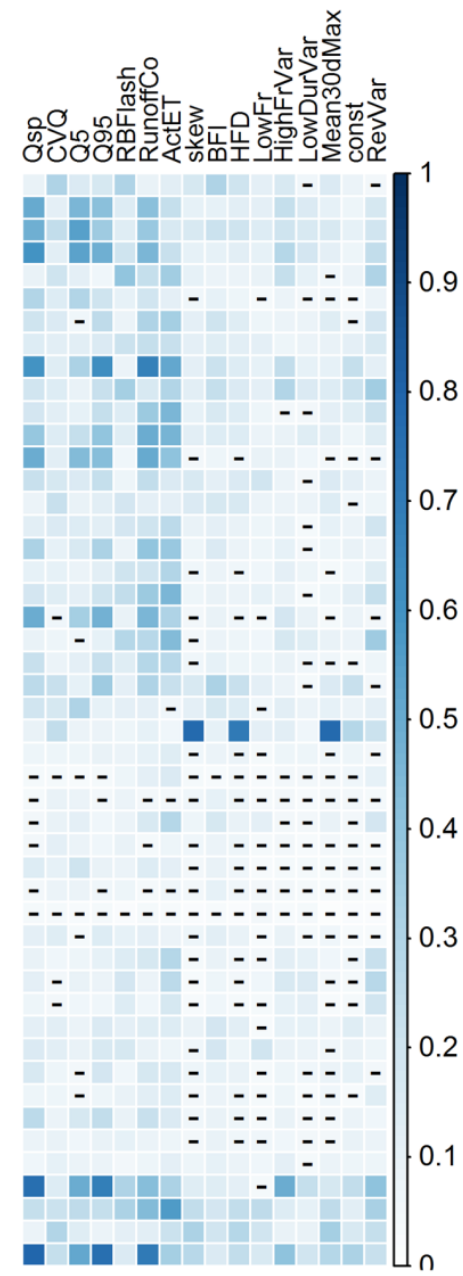

c. Distance correlation

Figure A: Correlation between catchment descriptors and flow signatures using a) Pearson, b) Spearman and c) distance correlations, respectively. Non-significant correlation according to the significance test are indicated with a dash (-).

\section{B. CART tree nodes description}

Table A: CART tree nodes description. For each node: number of flow stations affected to the node, percentage of stations affected to the node according to their original class from the FS classification. And total number of catchments affected to the node. The nodes are named after the affected class from the FS classification (1a, 1b, 1c are the nodes affected to class 1 , etc). The green boxes show the percentage of correctly classified gauges at each node.

For example, the node 1a contains 455 catchments of which 17 are gauged. Of these 17 gauges, 59\% were originally classified in class 1 of the classification based on flow signatures, $6 \%$ in class $3,29 \%$ in class 5 and $6 \%$ in class 6 . As a majority (59\%) of flow gauges at this node comes from class 1 of the FS classification, the node is affected to class 1.

\begin{tabular}{ccccccccccccccc}
\hline CART & Nb of & \multicolumn{1}{c}{ Repartition (\% of $\mathbf{n b}$ of gauges) of original classes } & Total No. \\
node & Gauges & $\mathbf{1}$ & $\mathbf{2}$ & $\mathbf{3}$ & $\mathbf{4}$ & $\mathbf{5}$ & $\mathbf{6}$ & $\mathbf{7}$ & $\mathbf{8}$ & $\mathbf{9}$ & $\mathbf{1 0}$ & $\mathbf{1 1}$ & catchments \\
\hline 1a & 17 & 59 & 0 & 6 & 0 & 29 & 6 & 0 & 0 & 0 & 0 & 0 & 455 \\
1b & 98 & 77 & 0 & 2 & 1 & 4 & 0 & 5 & 0 & 5 & 4 & 2 & 6423 \\
1c & 9 & 56 & 0 & 0 & 0 & 33 & 0 & 0 & 11 & 0 & 0 & 0 & 811 \\
3a & 492 & 2 & 0 & 35 & 7 & 14 & 5 & 0 & 16 & 13 & 1 & 7 & 13645 \\
3b & 29 & 0 & 0 & 48 & 0 & 10 & 7 & 0 & 21 & 7 & 0 & 7 & 424 \\
3c & 18 & 0 & 0 & 50 & 0 & 6 & 6 & 0 & 17 & 11 & 0 & 11 & 213 \\
3d & 20 & 10 & 0 & 40 & 15 & 0 & 10 & 0 & 10 & 10 & 0 & 5 & 177
\end{tabular}




\begin{tabular}{cccccccccccccc}
$\mathbf{4}$ & 73 & 1 & 6 & 10 & 53 & 4 & 3 & 3 & 12 & 7 & 0 & 1 & 5112 \\
$\mathbf{5 a}$ & 33 & 0 & 0 & 6 & 0 & 64 & 0 & 0 & 6 & 18 & 0 & 6 & 932 \\
$\mathbf{5 b}$ & 43 & 0 & 0 & 9 & 0 & 88 & 0 & 0 & 0 & 2 & 0 & 0 & 833 \\
$\mathbf{6 a}$ & 17 & 0 & 0 & 0 & 0 & 0 & 53 & 6 & 0 & 29 & 0 & 12 & 228 \\
$\mathbf{6 b}$ & 14 & 0 & 0 & 14 & 14 & 0 & 57 & 0 & 14 & 0 & 0 & 0 & 138 \\
$\mathbf{6 c}$ & 202 & 3 & 0 & 3 & 2 & 0 & 53 & 10 & 10 & 7 & 5 & 6 & 1971 \\
$\mathbf{7}$ & 33 & 3 & 0 & 0 & 0 & 0 & 12 & 70 & 0 & 3 & 6 & 6 & 678 \\
$\mathbf{8}$ & 64 & 2 & 0 & 6 & 8 & 0 & 20 & 5 & 45 & 8 & 2 & 5 & 670 \\
$\mathbf{9 a}$ & 10 & 10 & 0 & 0 & 0 & 20 & 0 & 0 & 0 & 70 & 0 & 0 & 200 \\
$\mathbf{9 b}$ & 48 & 4 & 0 & 6 & 8 & 13 & 0 & 0 & 0 & 67 & 0 & 2 & 769 \\
$\mathbf{1 0}$ & 81 & 0 & 0 & 1 & 0 & 0 & 11 & 17 & 3 & 3 & 57 & 9 & 762 \\
$\mathbf{1 1 a}$ & 15 & 0 & 0 & 0 & 0 & 13 & 0 & 0 & 0 & 0 & 0 & 87 & 110 \\
$\mathbf{1 1 b}$ & 10 & 0 & 0 & 0 & 0 & 10 & 0 & 10 & 0 & 0 & 0 & 80 & 146 \\
$\mathbf{1 1 c}$ & 40 & 15 & 0 & 0 & 0 & 0 & 8 & 3 & 5 & 5 & 10 & 55 & 518 \\
\hline
\end{tabular}




\section{Material for the analysis of the CART classification (base material for table 3)}

\section{C.1 Flow signatures}

C.1.1 Distribution (boxplots) of flow signatures in the different classes
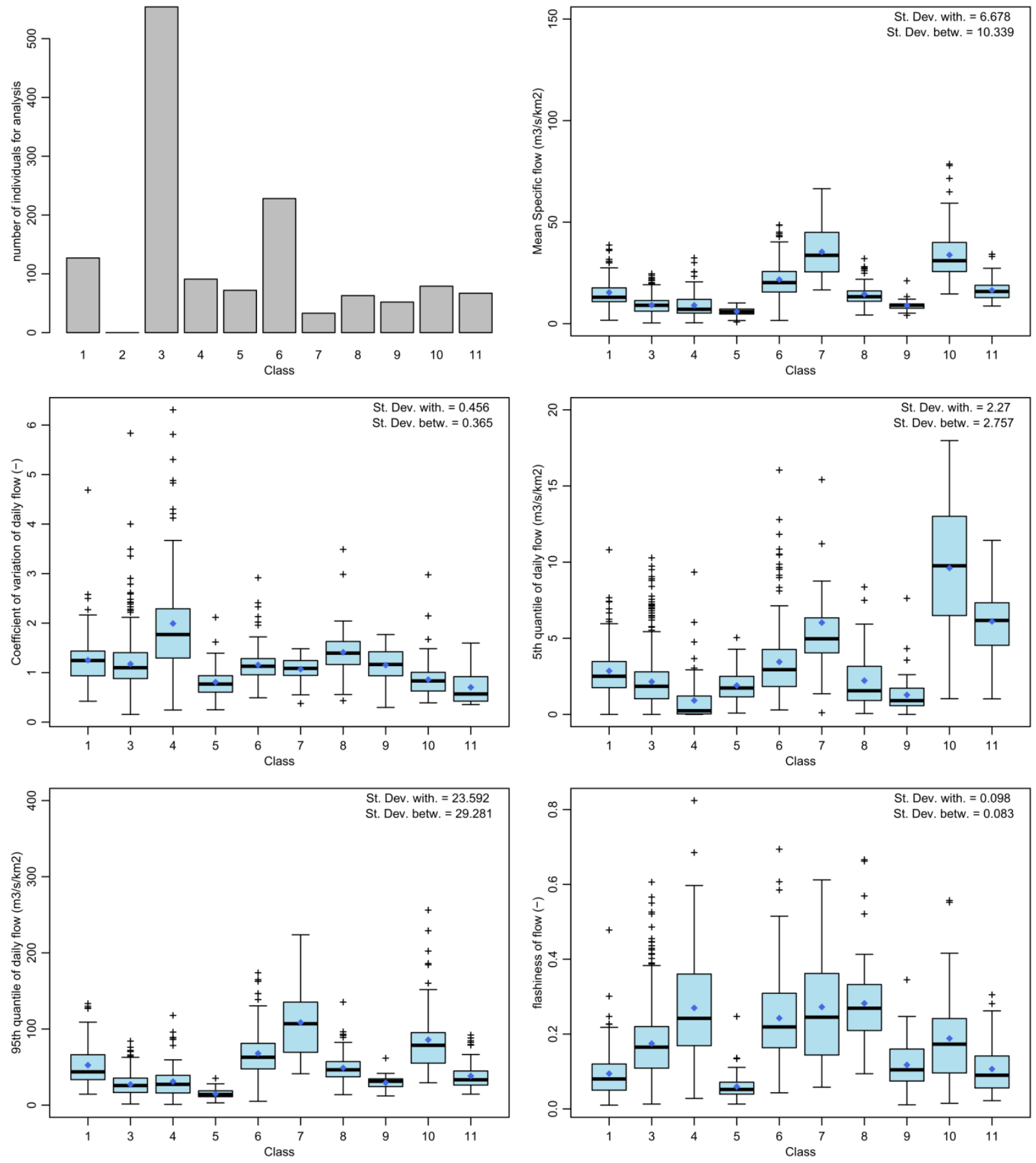

Figure B: boxplots of flow signatures in the different classes of the CART classification (1/3). 

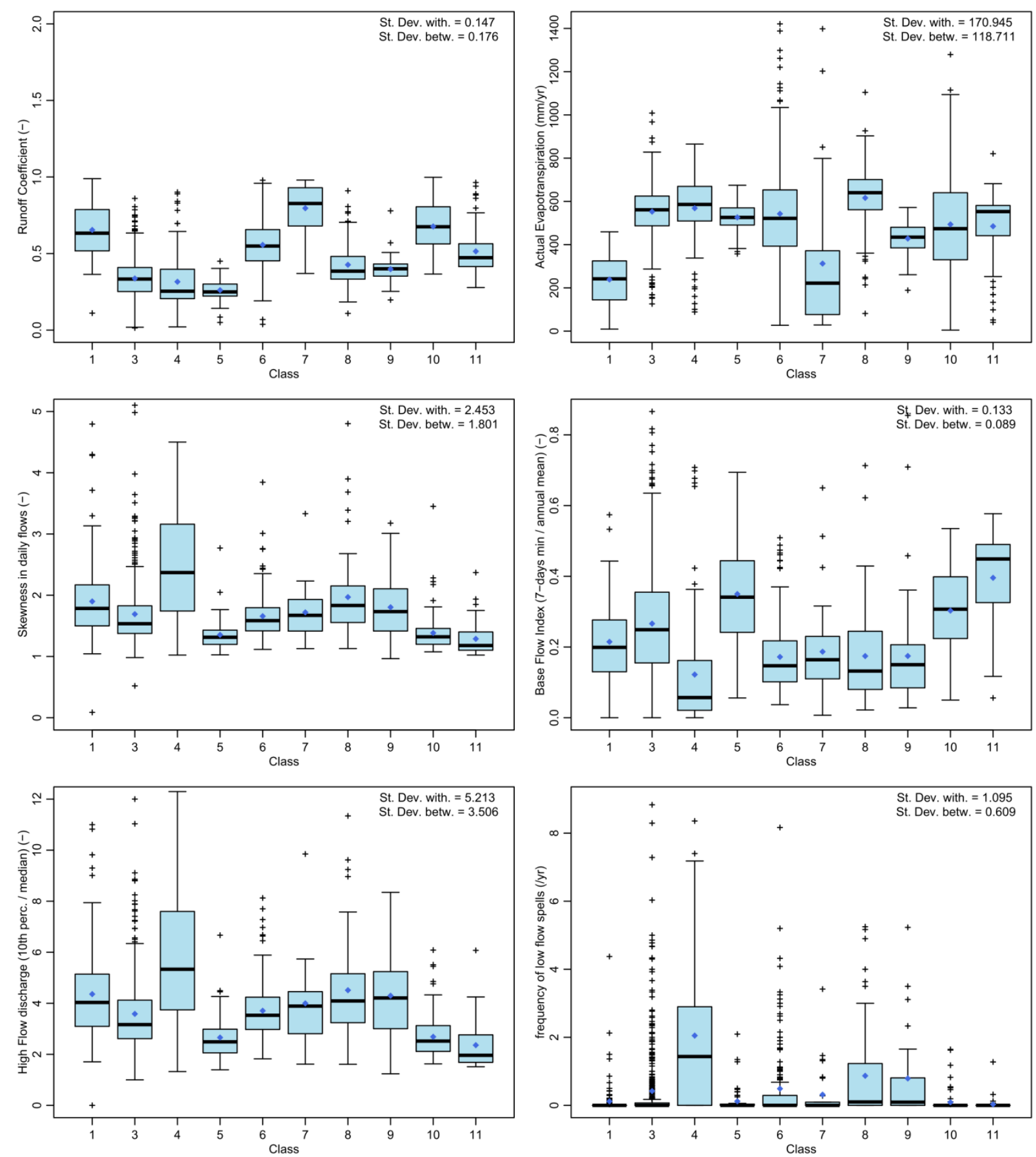

Figure C: boxplots of flow signatures in the different classes of the CART classification (2/3). 

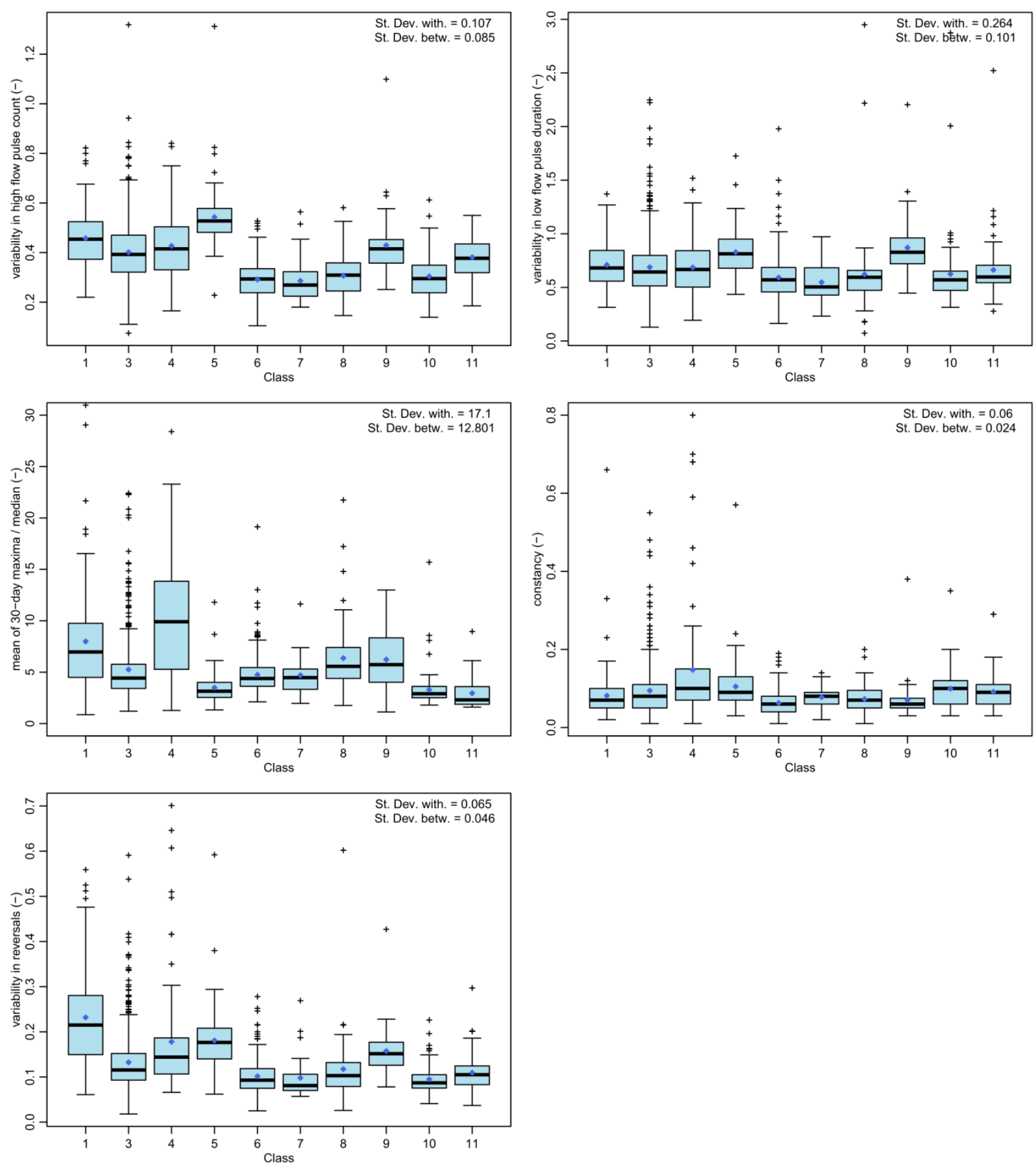

Figure D: boxplots of flow signatures in the different classes of the CART classification (3/3). 


\section{C.1.2 Matrix of median flow signatures for each class compared to the whole set of stream gauges}

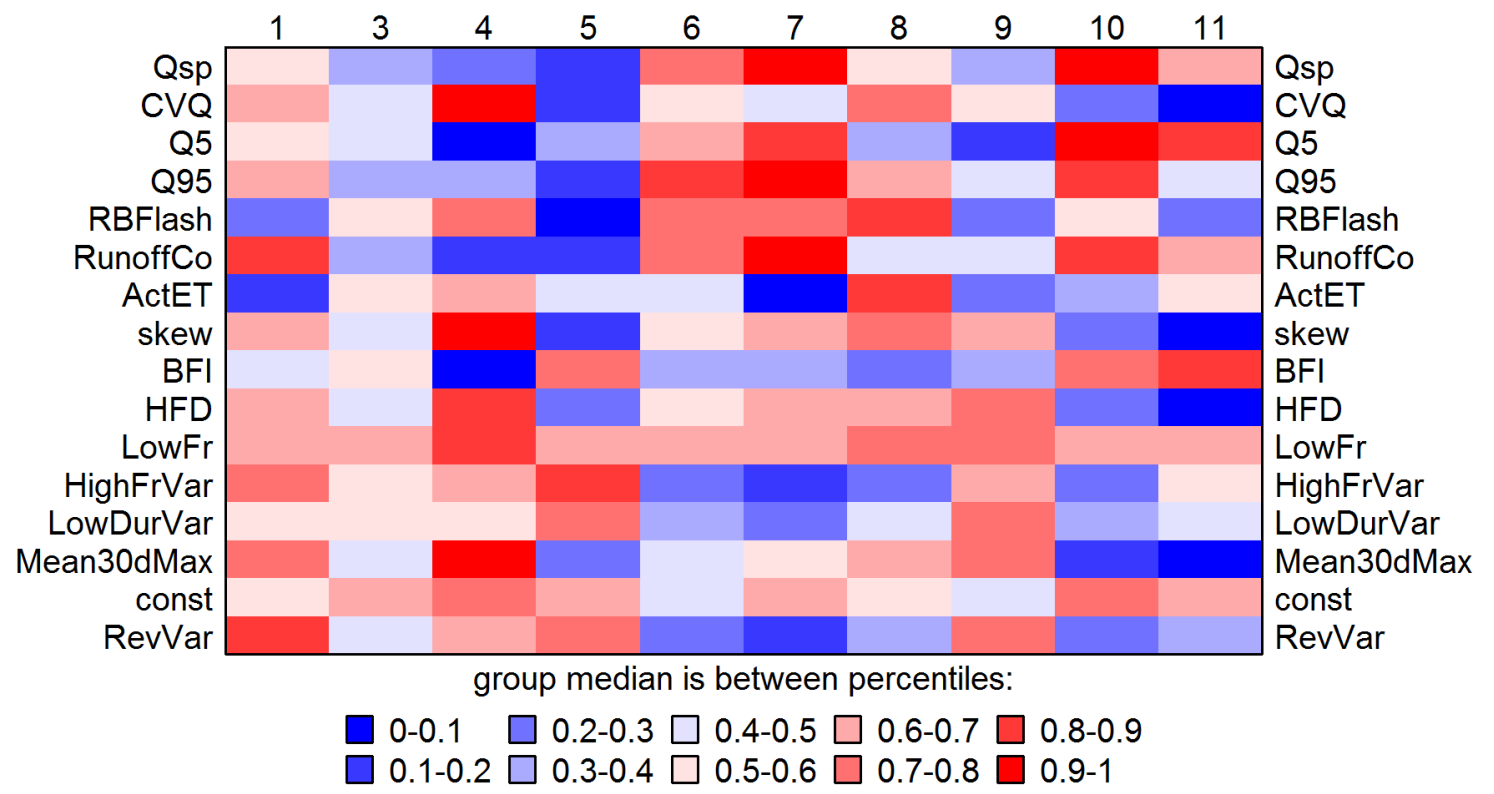

Figure E: Visualization of median flow signatures in each class of the CART classification - Position of the class median flow signature in the distribution (percentiles) of the same flow signature among the whole set of gauges. 


\section{C.2 Catchment descriptors}

C.2.1 Distribution (boxplots) of catchment descriptors in the different classes
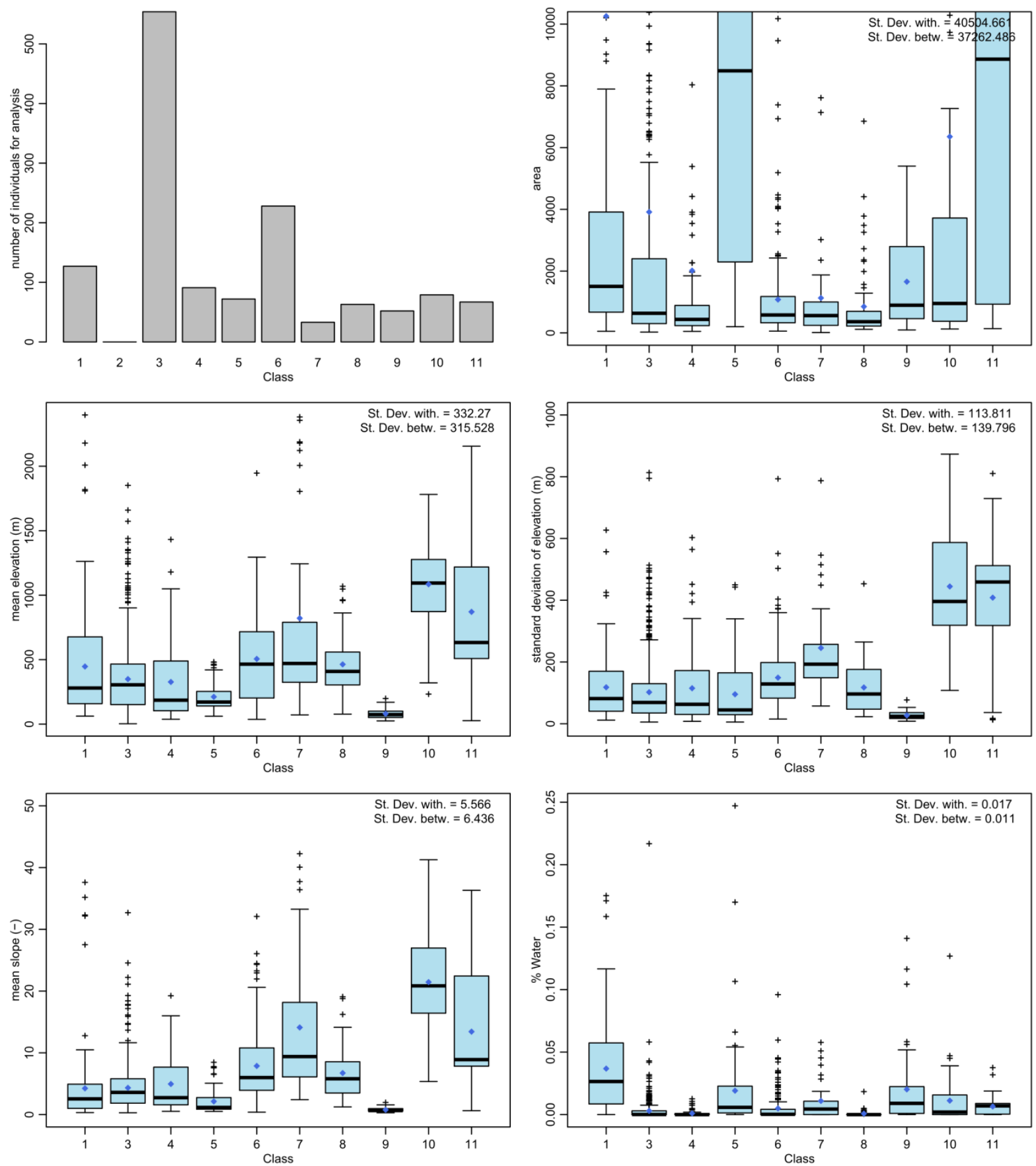

Figure F: boxplots of catchment descriptors in the different classes of the CART classification (1/7). 

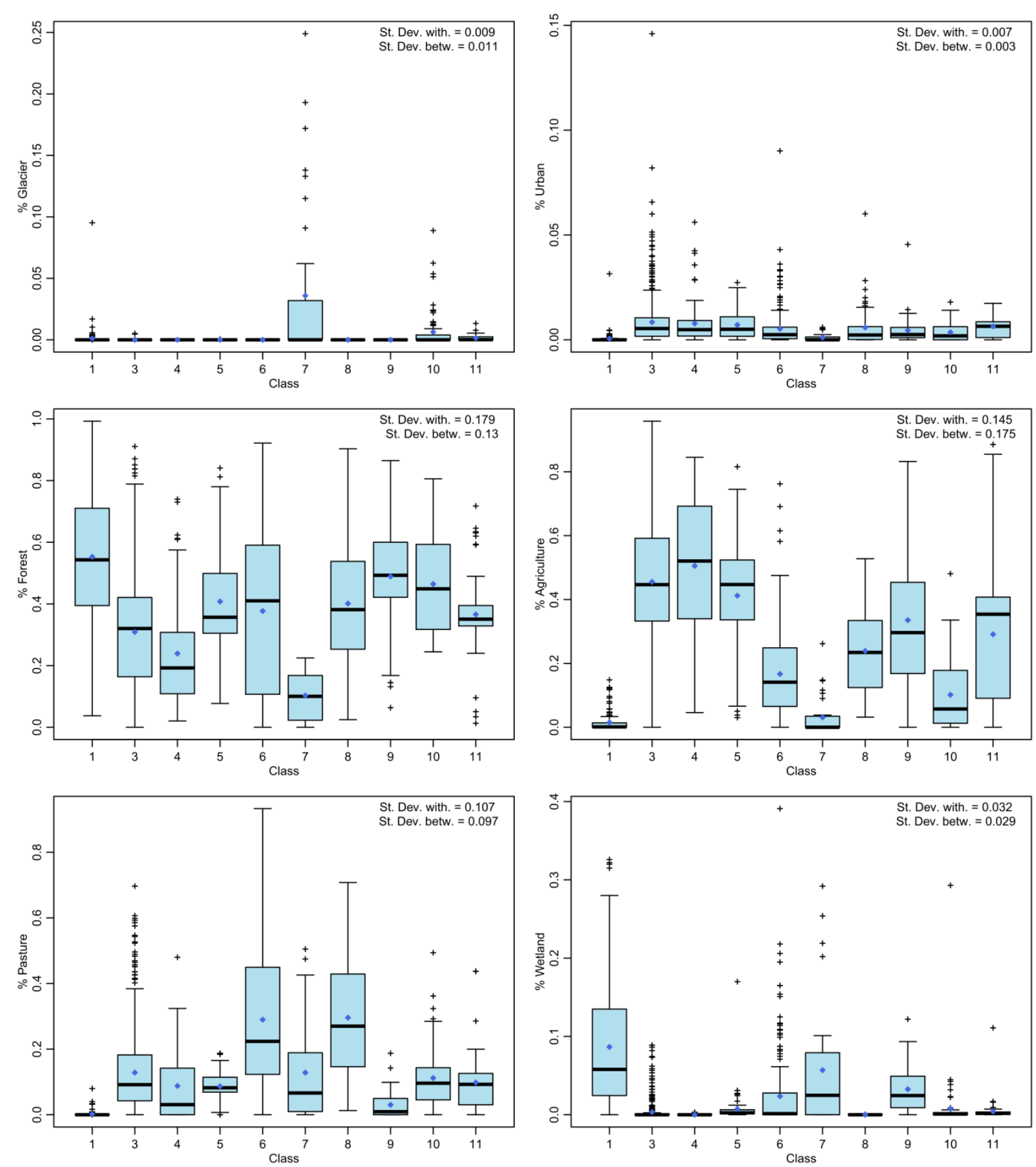

Figure G: boxplots of catchment descriptors in the different classes of the CART classification (2/7). 

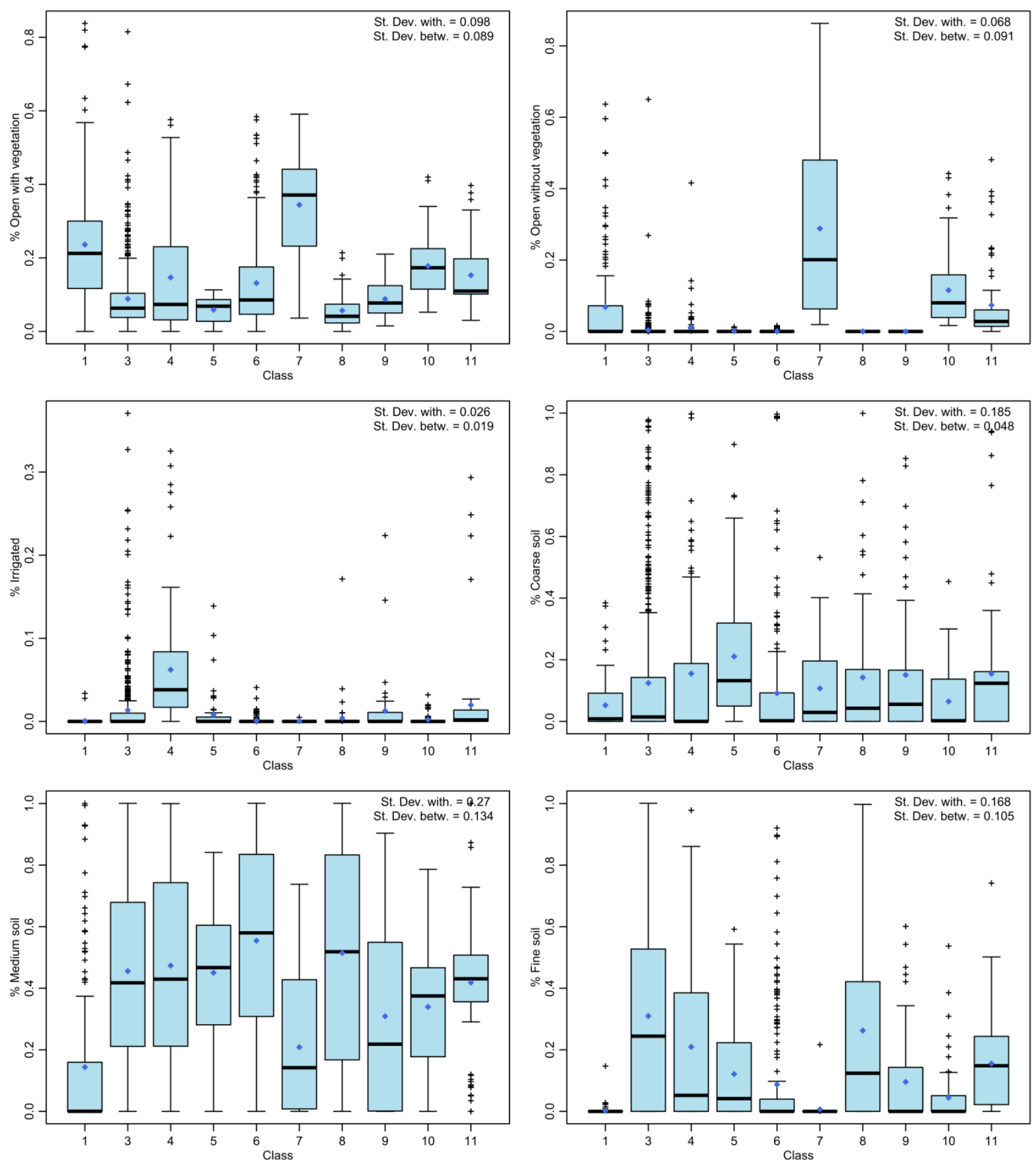

Figure H: boxplots of catchment descriptors in the different classes of the CART classification (3/7). 

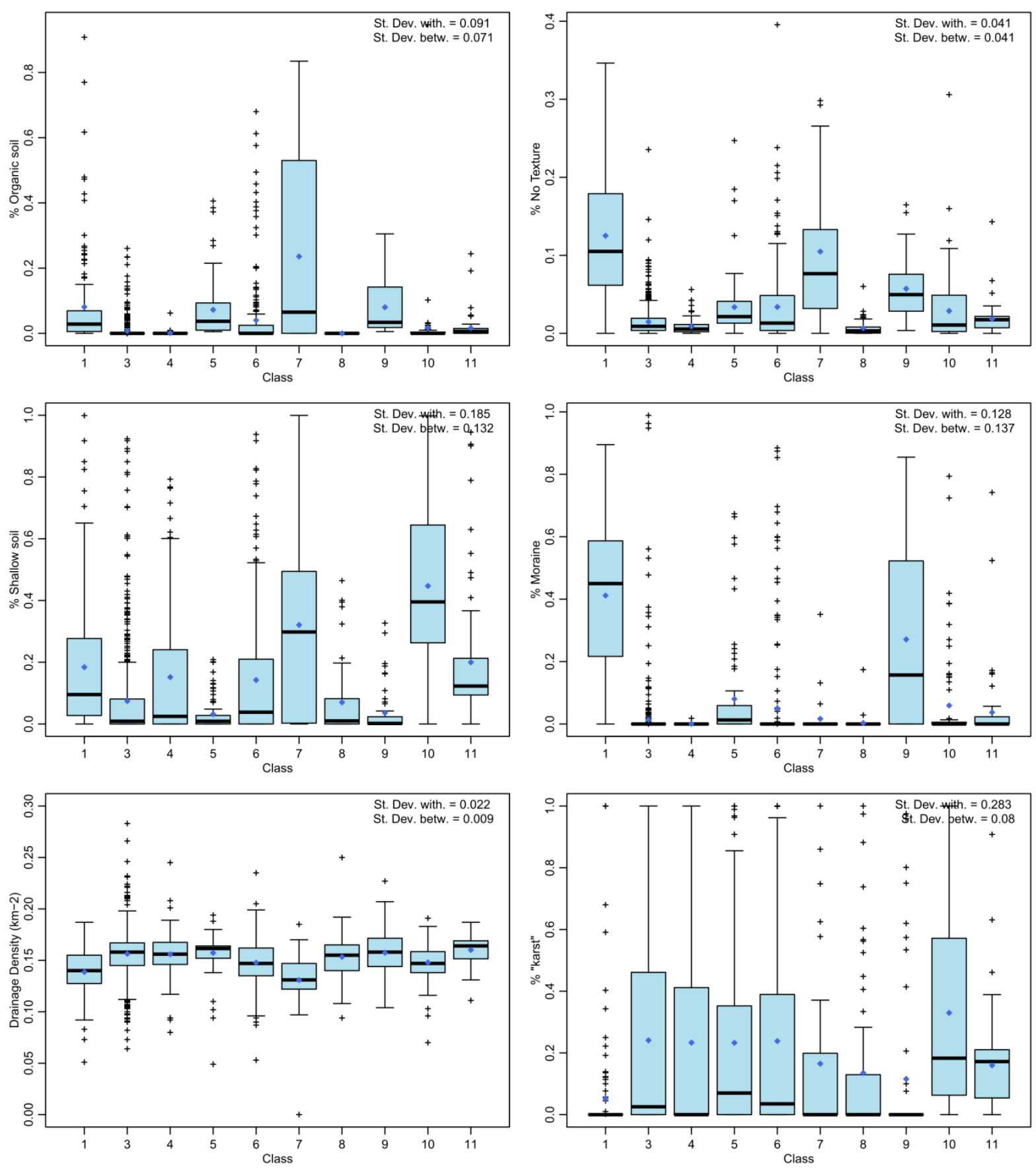

Figure I: boxplots of catchment descriptors in the different classes of the CART classification (4/7). 

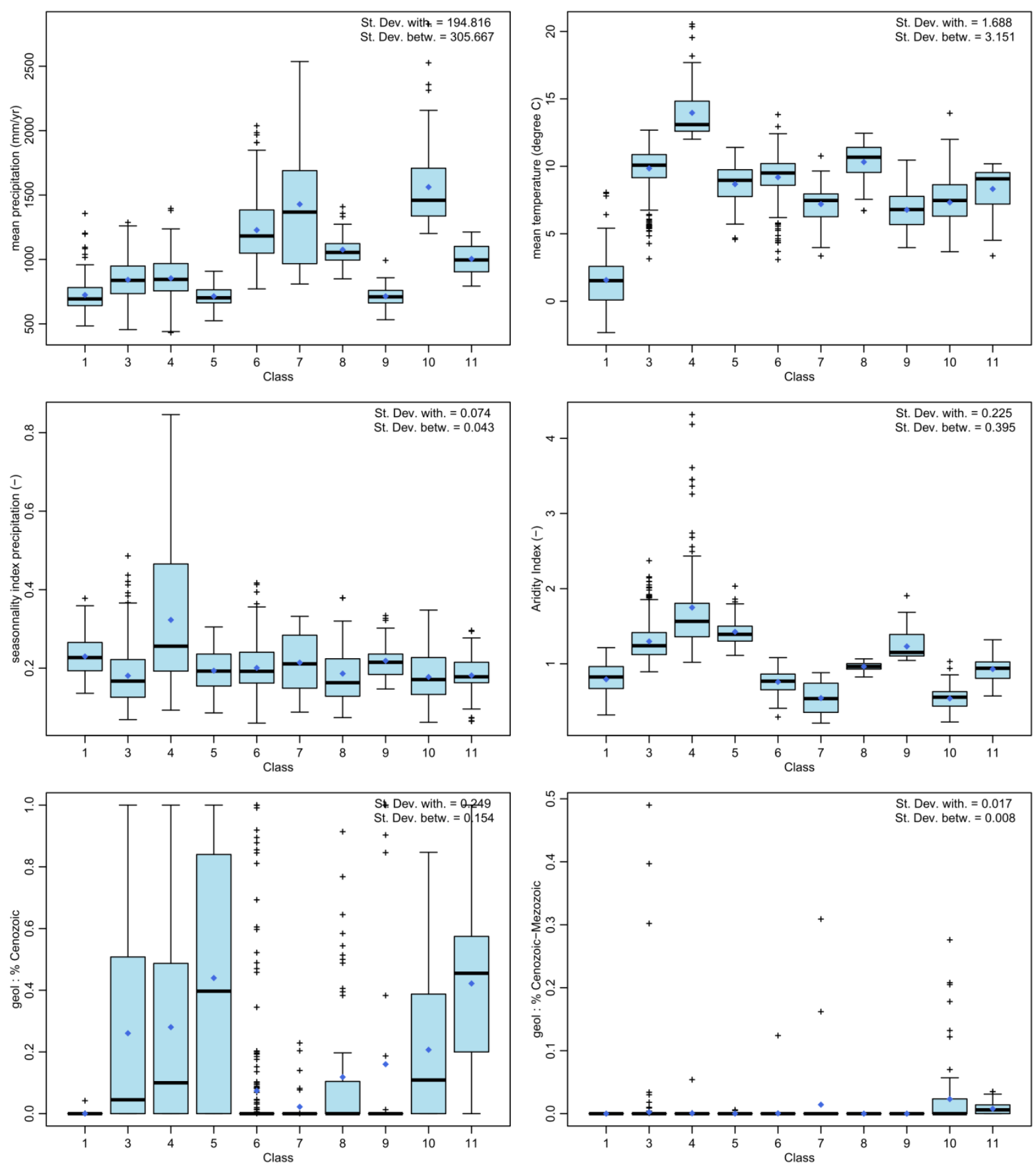

Figure J: boxplots of catchment descriptors in the different classes of the CART classification (5/7). 

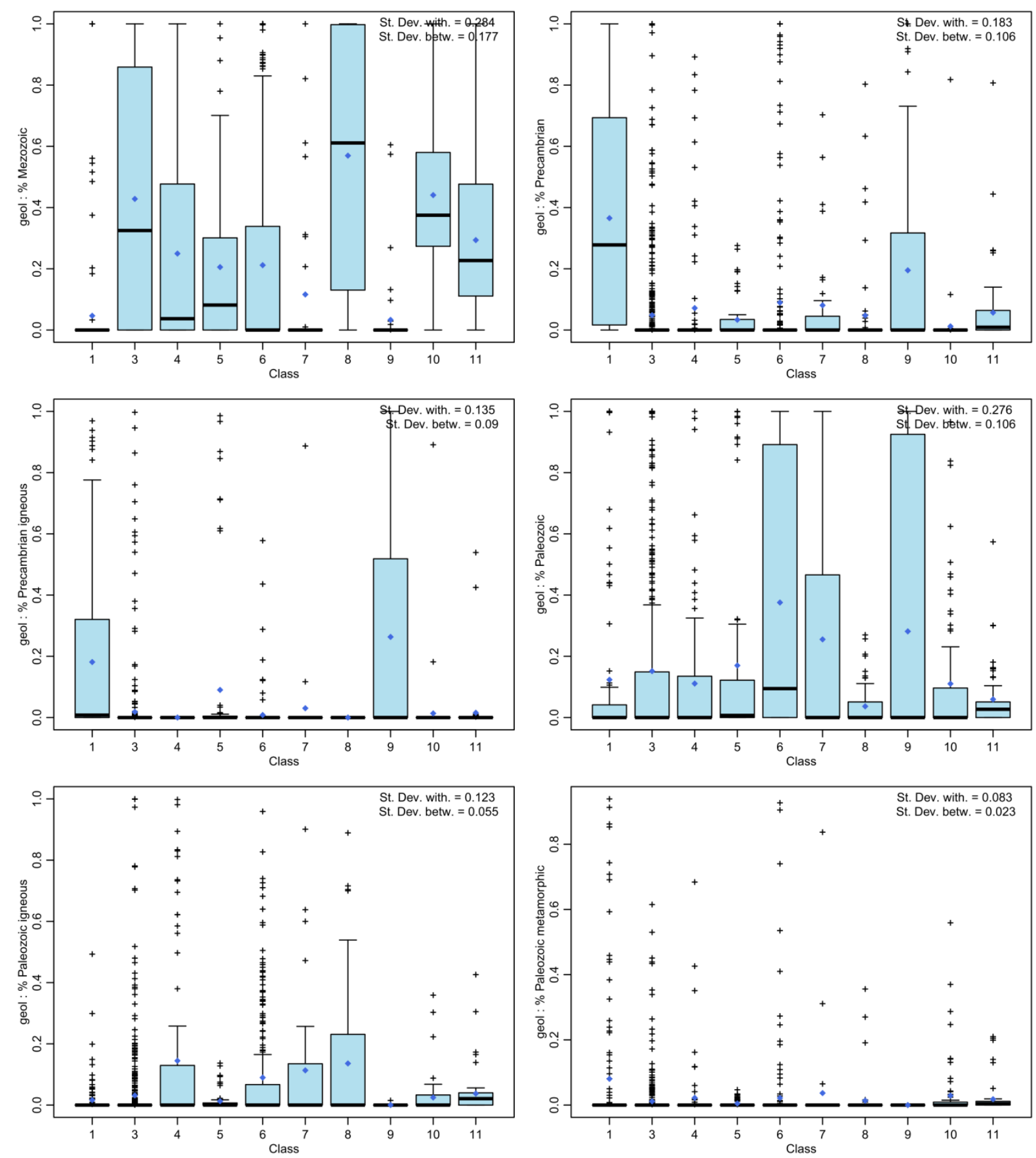

Figure K: boxplots of catchment descriptors in the different classes of the CART classification (6/7). 


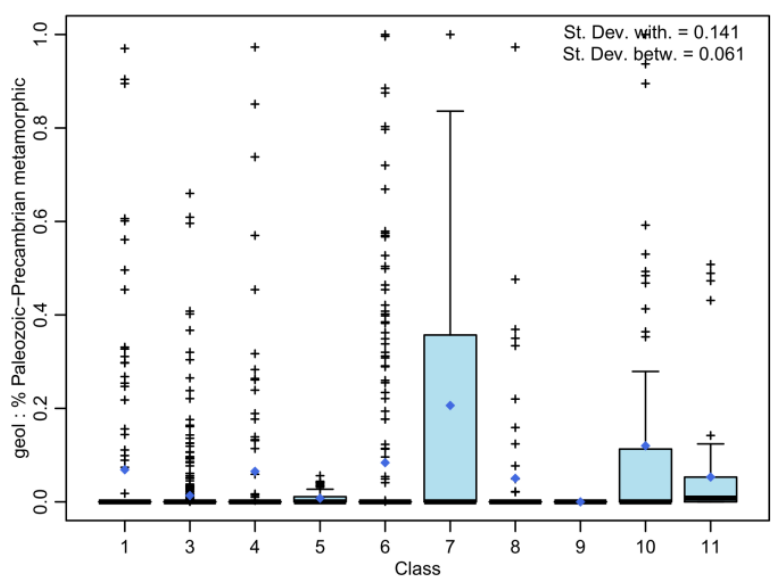

Figure L: boxplots of catchment descriptors in the different classes of the CART classification (7/7). 


\section{C.2.2 Matrix of median catchment descriptors for each class compared to the whole set of stream gauges / catchments}

a. Characteristics of catchments with stream gauges (1366)

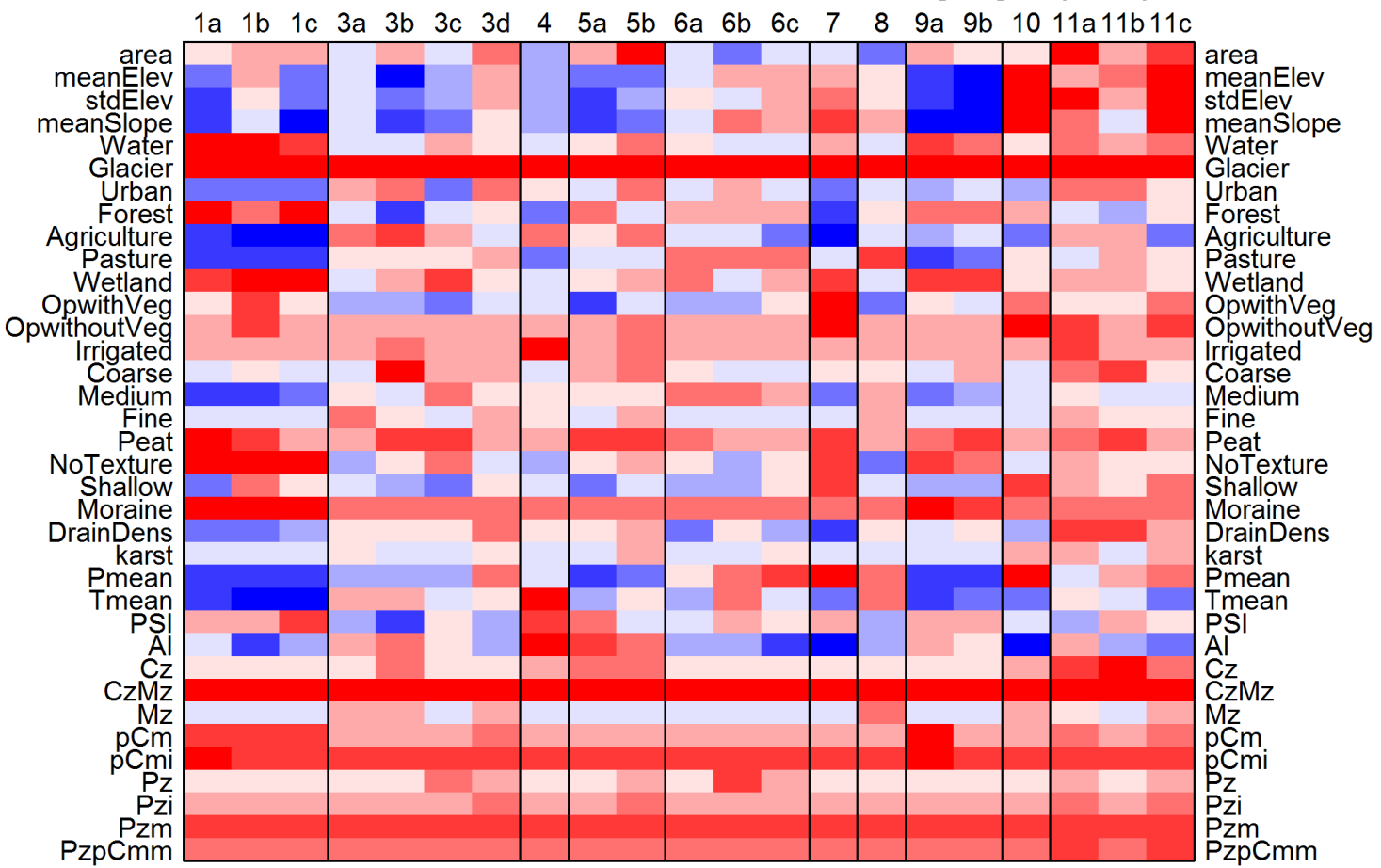

b. Characteristics of all catchments (35215)

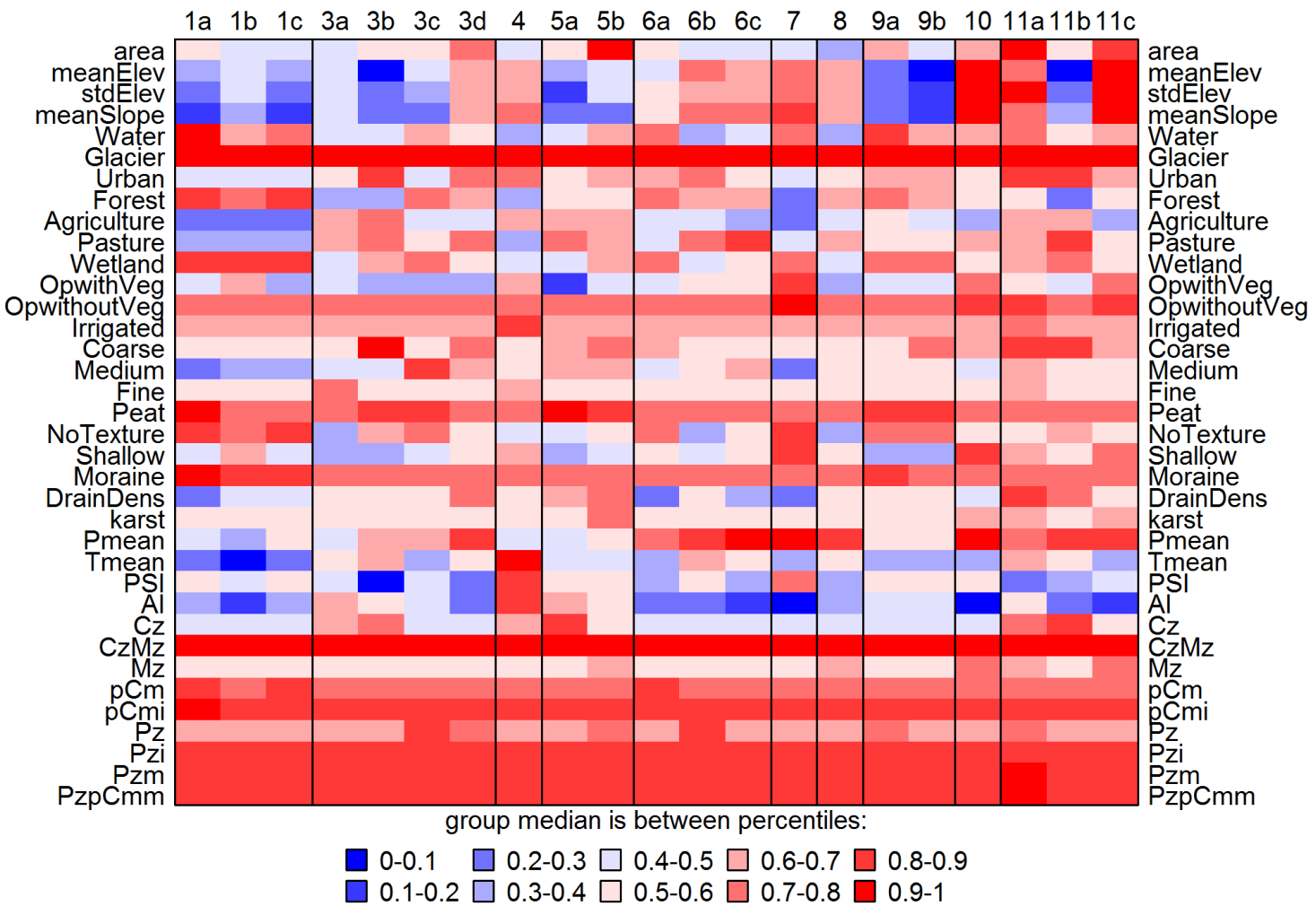

Figure M: Visualization of median catchment descriptors in each class of the CART classification - Position of the class median catchment descriptor in the distribution (percentiles) of the same catchment descriptor among the whole set of gauges (a)/catchments (b). Plot a is built when looking at only gauged catchments while plot $b$ is built using all 35215 classified catchments. When more than one node in the CART tree (fig. 6 of the main article) is affected to a given class, the detailed characteristics of each node are shown in the figure. 
C.3 Larger map of the classification and detailed maps for classes corresponding to more than one node in the CART tree

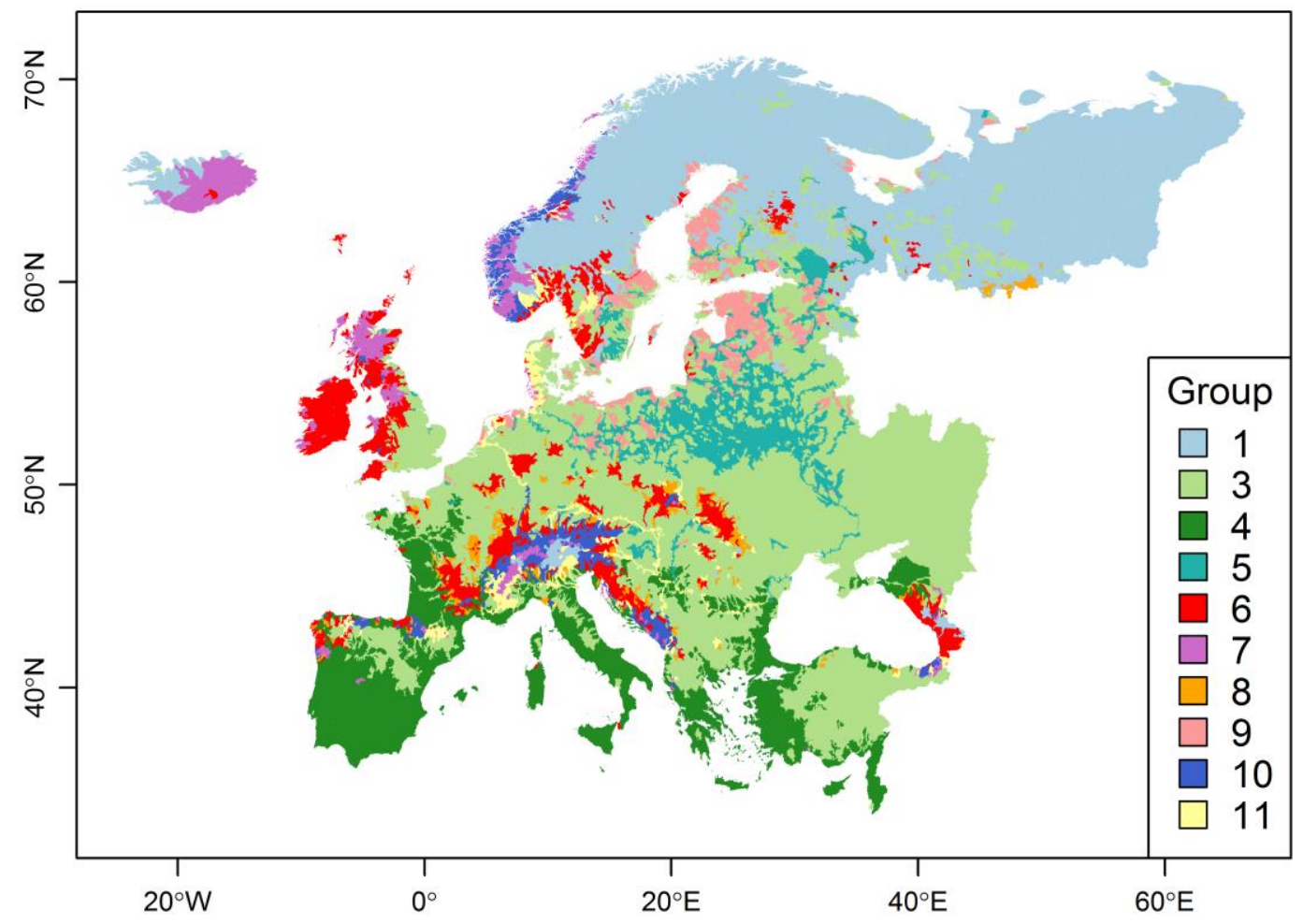

Figure N: Map of the CART classification 

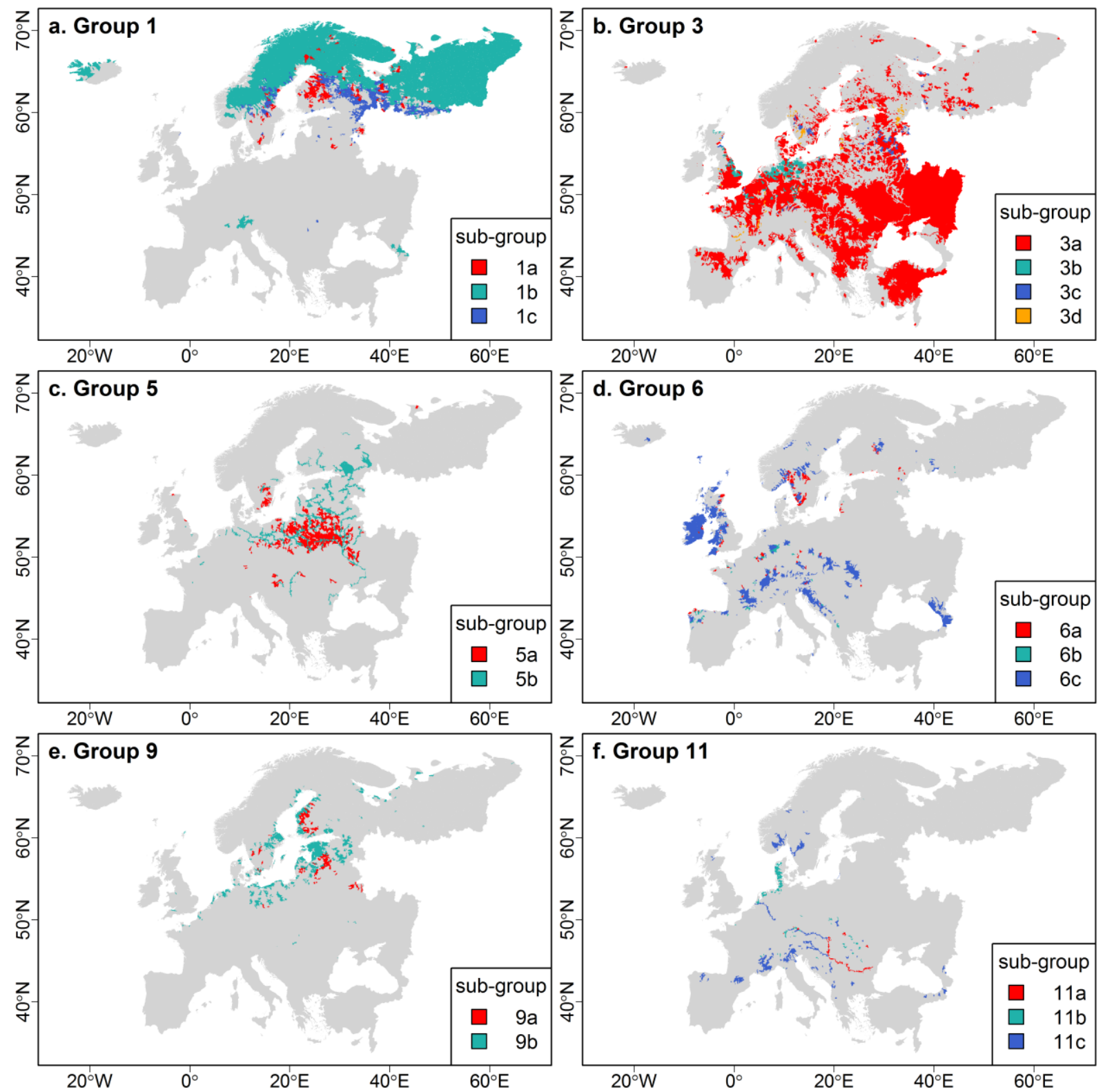

Figure 0: detailed maps for classes corresponding to more than one node in the CART tree. The names $1 \mathrm{a}, 1 \mathrm{~b}, 1 \mathrm{c}$ etc are the names of the nodes shown in Fig. 6 of the main article and table 1 of the present supplementary material. 
D. Distribution (boxplots) of catchment descriptors and flow signatures in the different classes of FS and CD classifications (plots are commented in section 3.2)

D.1 Classification based on flow signatures (FS classification)

D.1.1 Boxplots of flow signatures
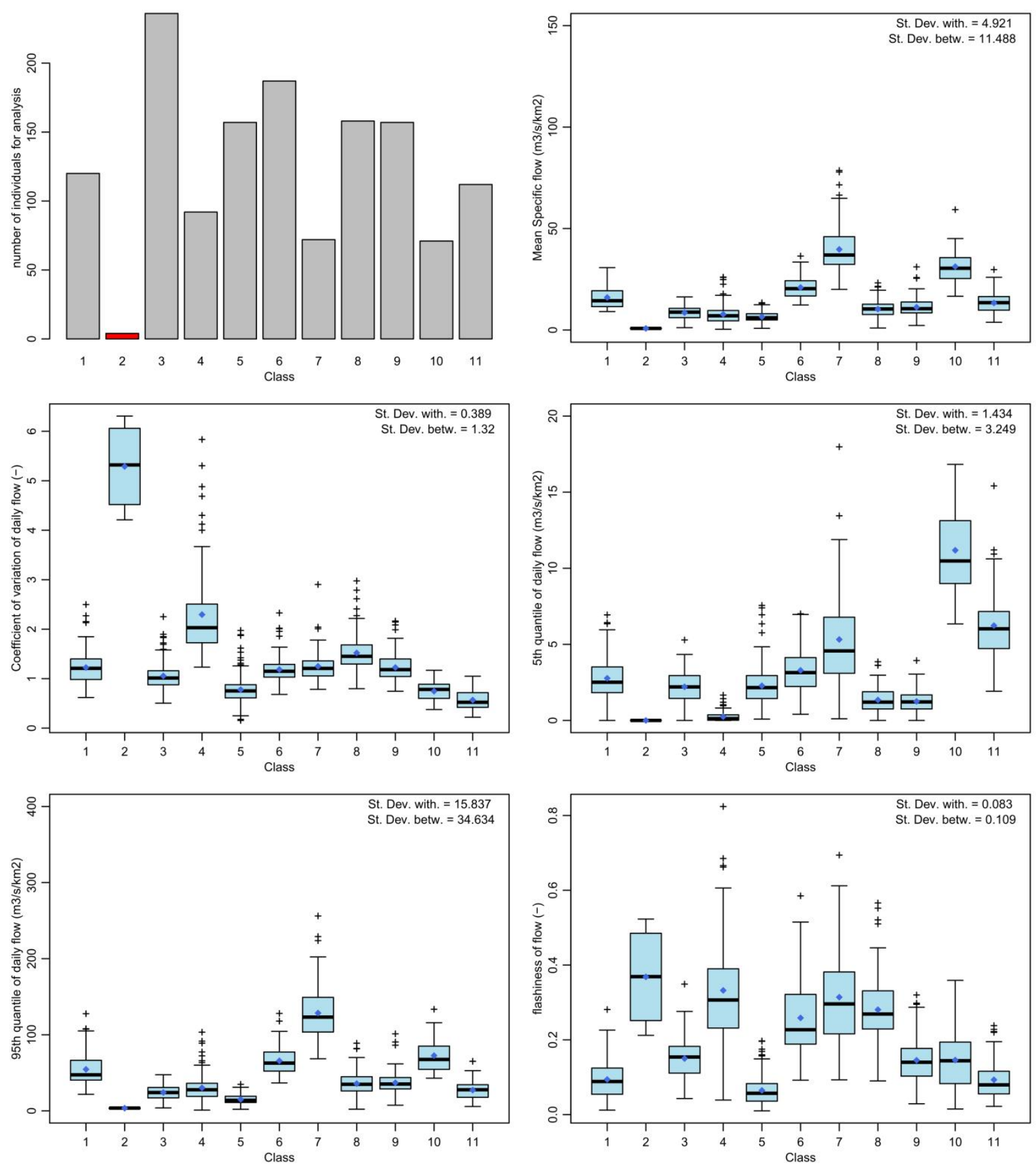

Figure P: boxplots of flow signatures in the different classes of the FS classification (1/3) 

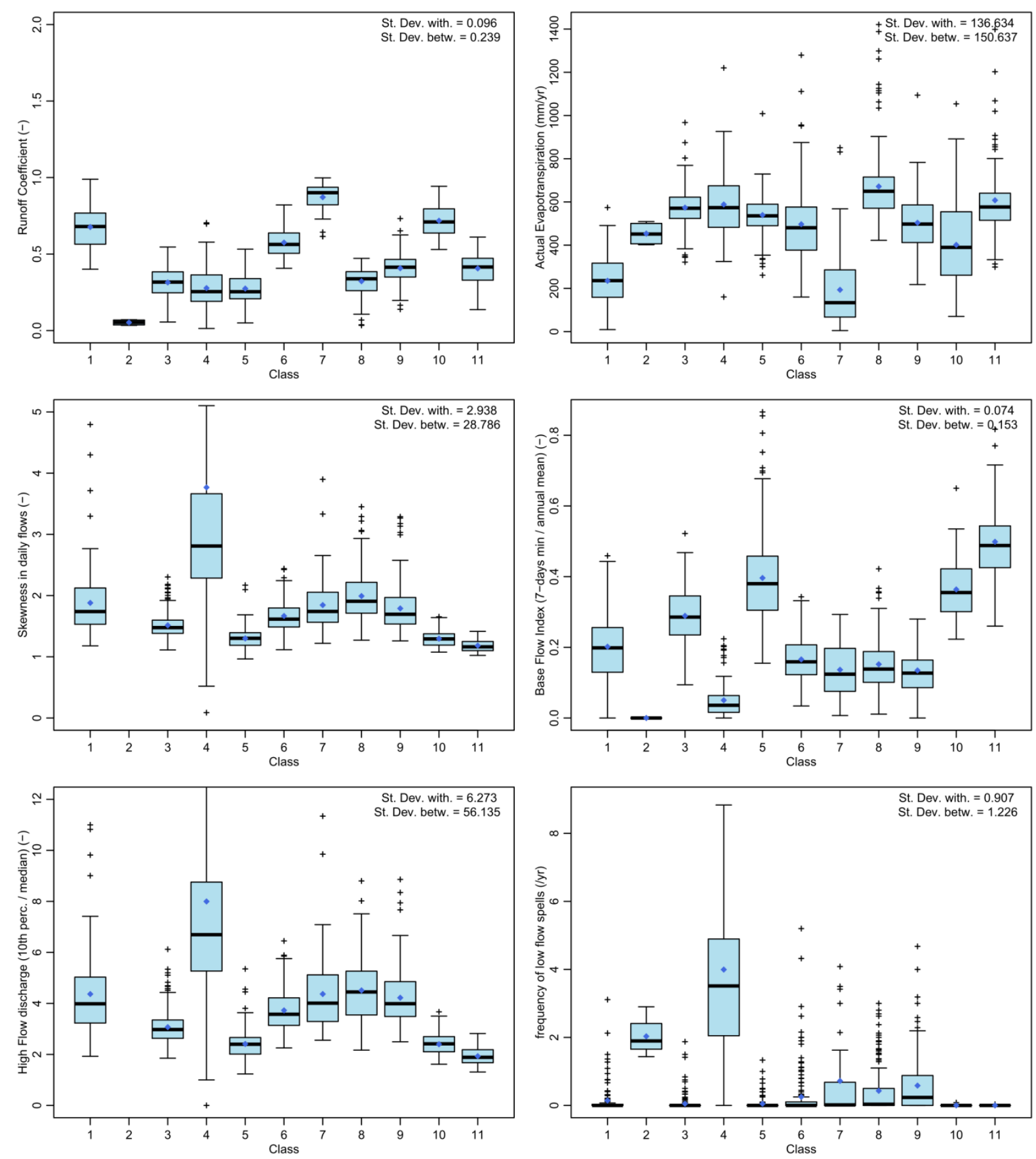

Figure Q: boxplots of flow signatures in the different classes of the FS classification (2/3) 

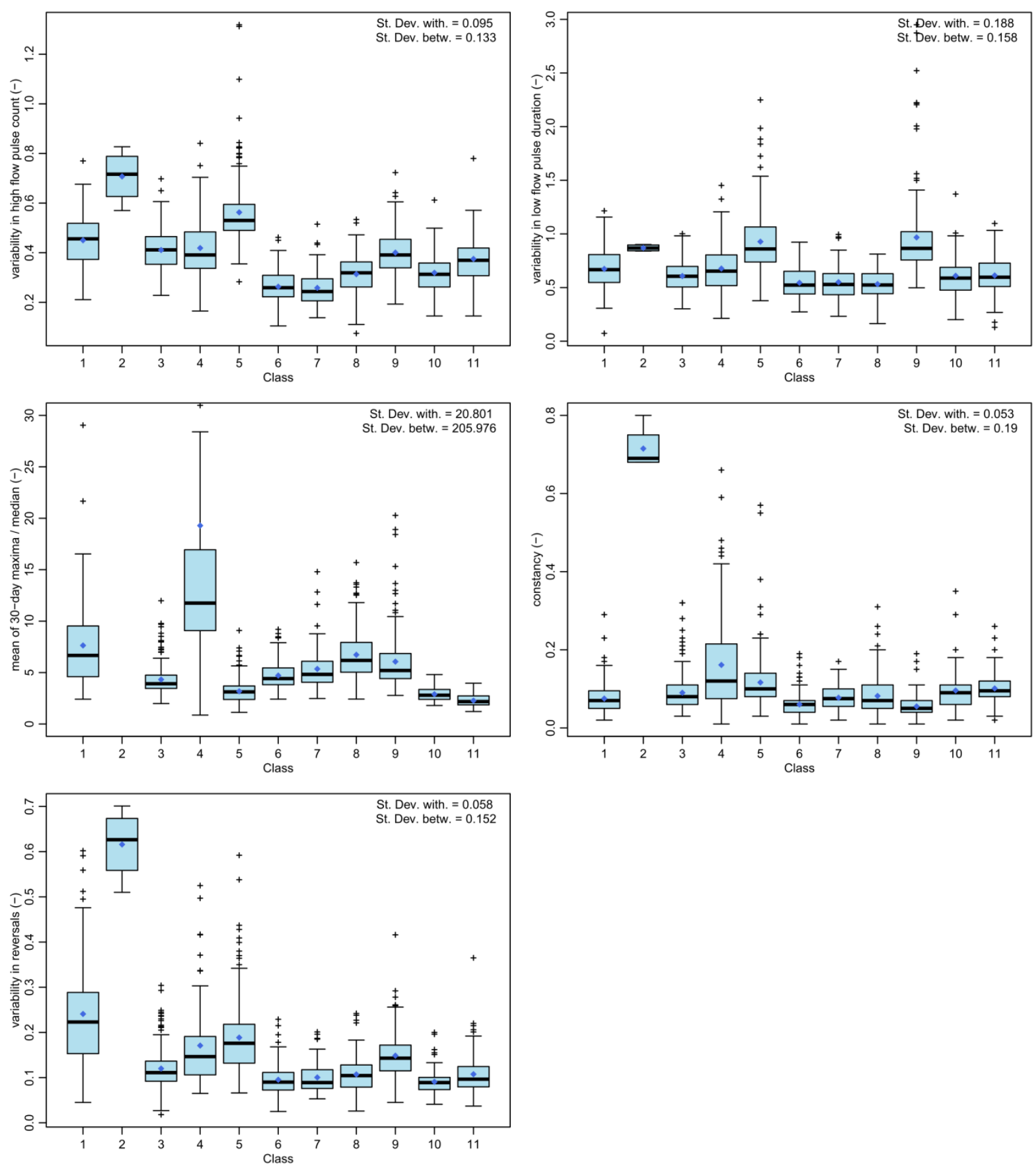

Figure R: boxplots of flow signatures in the different classes of the FS classification (3/3) 


\section{D.1.2 Boxplots of catchment descriptors}
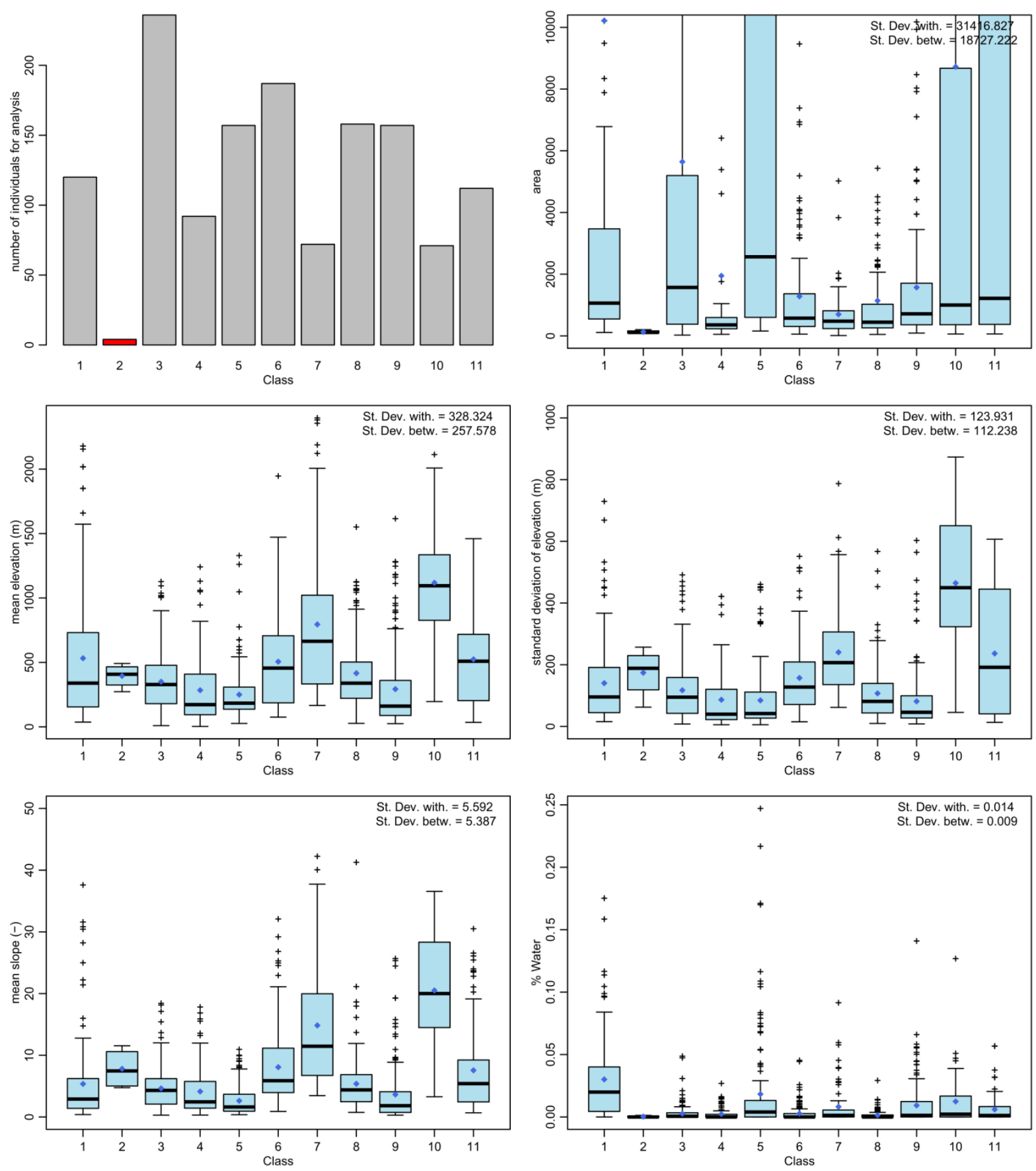

Figure S: boxplots of catchment descriptors in the different classes of the FS classification (1/7) 

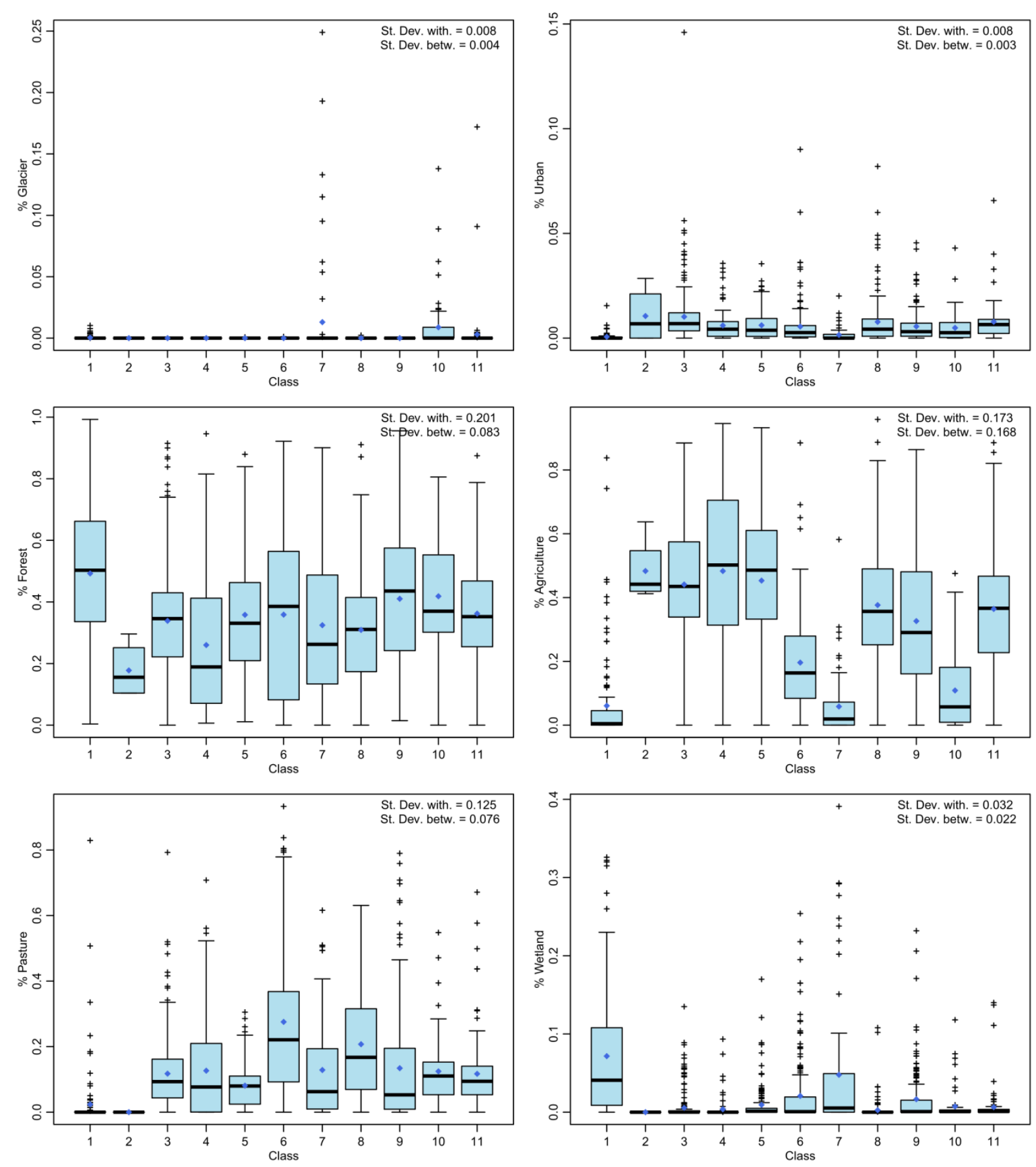

Figure T: boxplots of catchment descriptors in the different classes of the FS classification (2/7) 

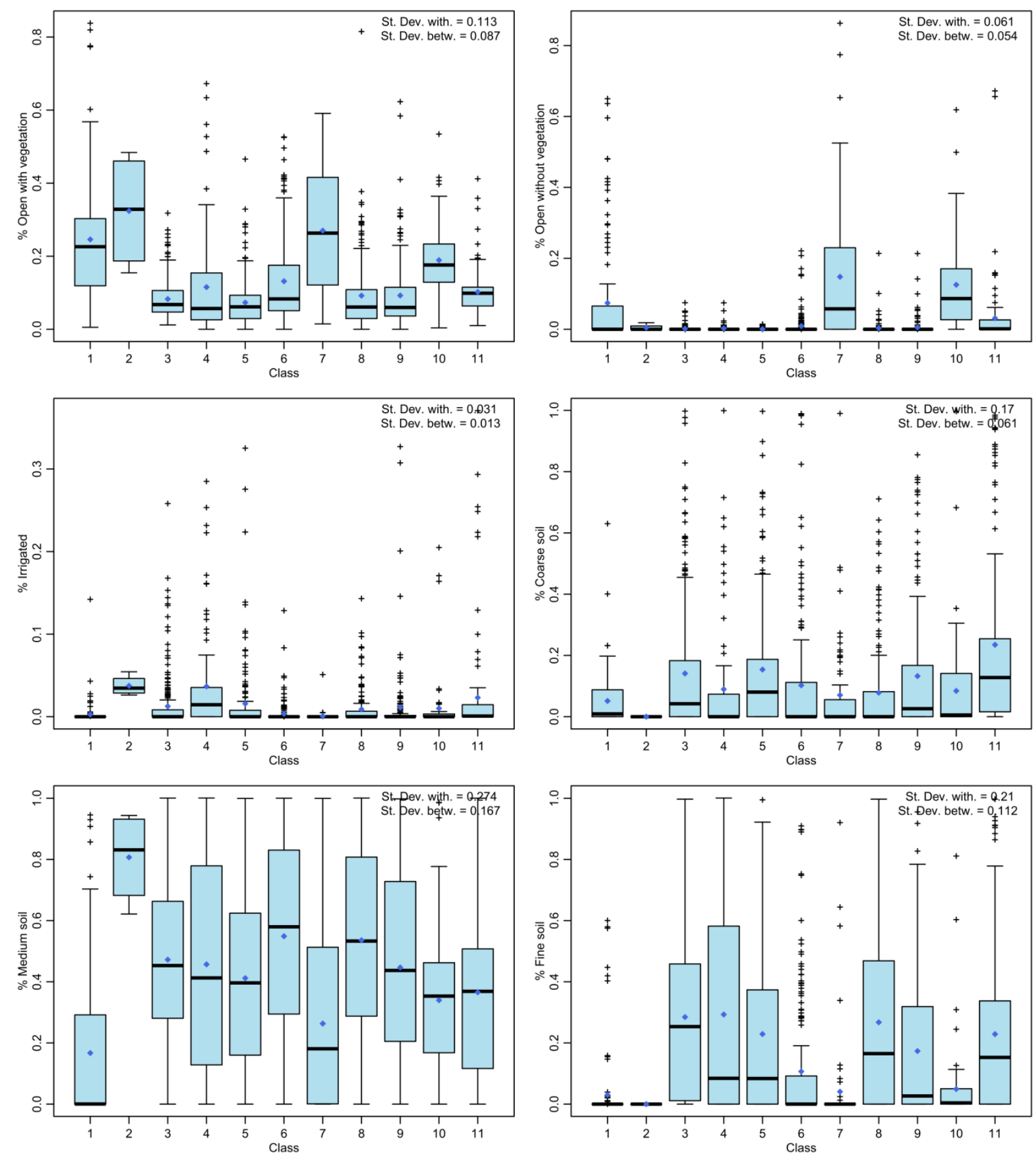

Figure U: boxplots of catchment descriptors in the different classes of the FS classification (3/7) 

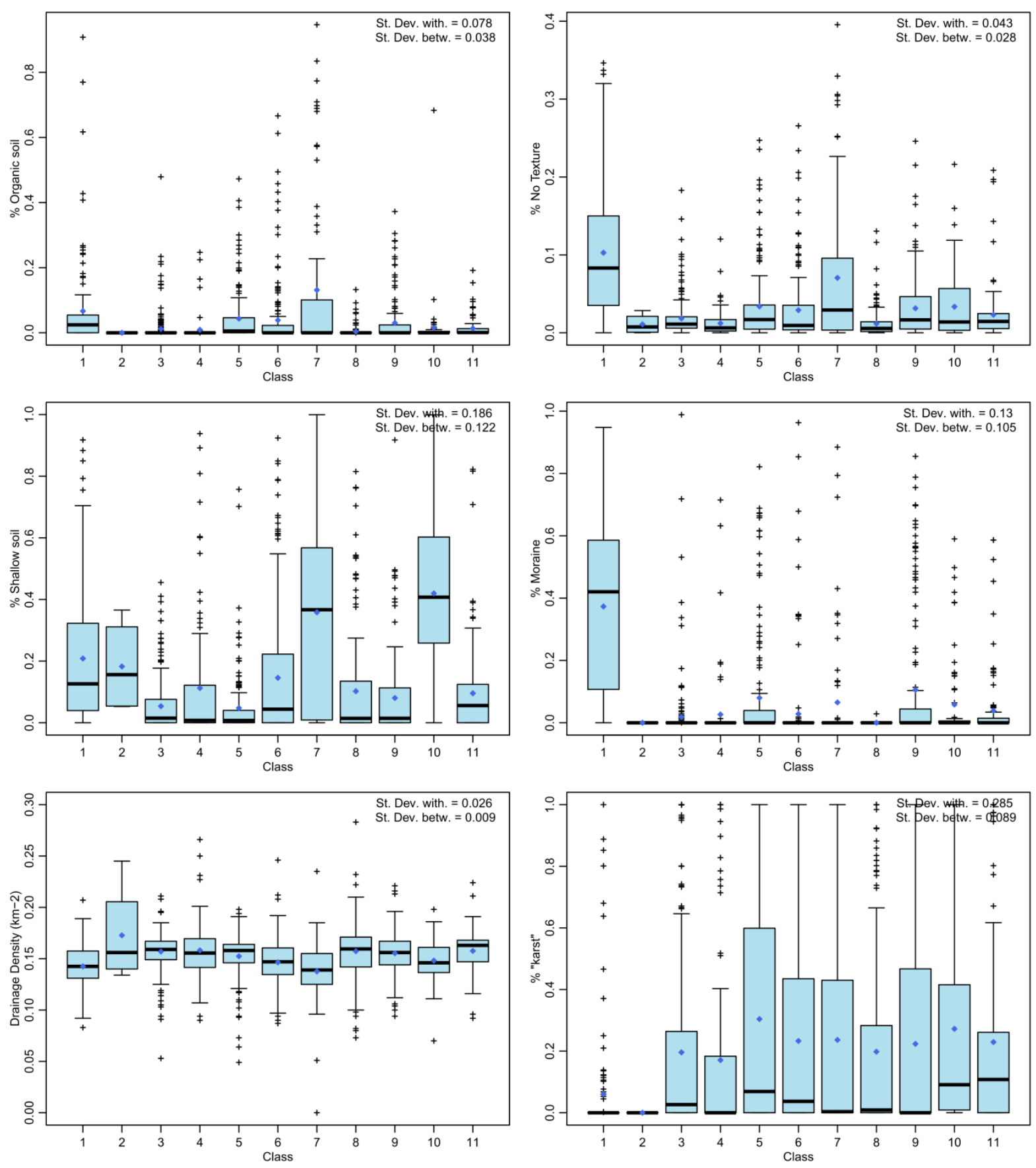

Figure V: boxplots of catchment descriptors in the different classes of the FS classification (4/7) 

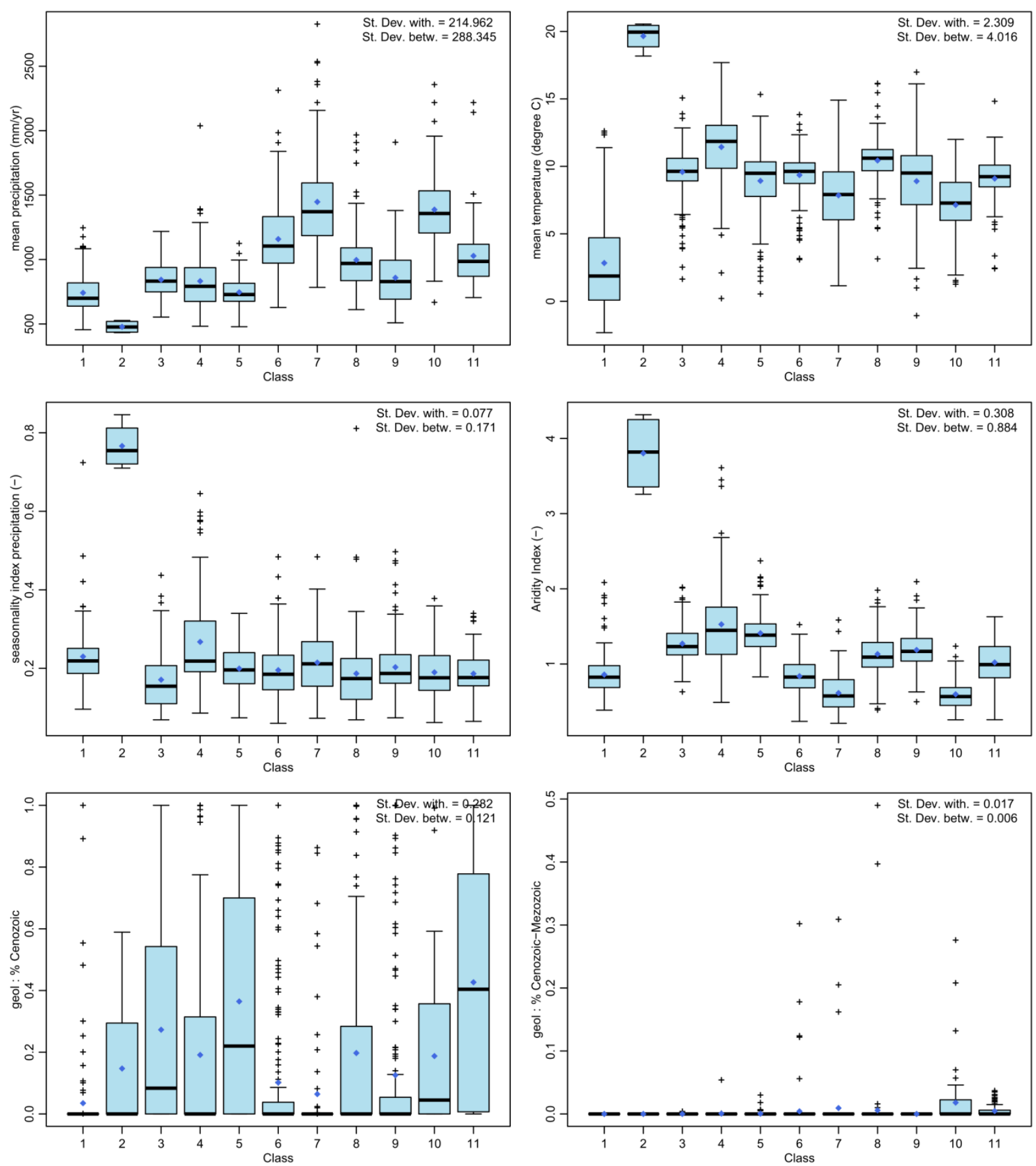

Figure W: boxplots of catchment descriptors in the different classes of the FS classification (5/7) 

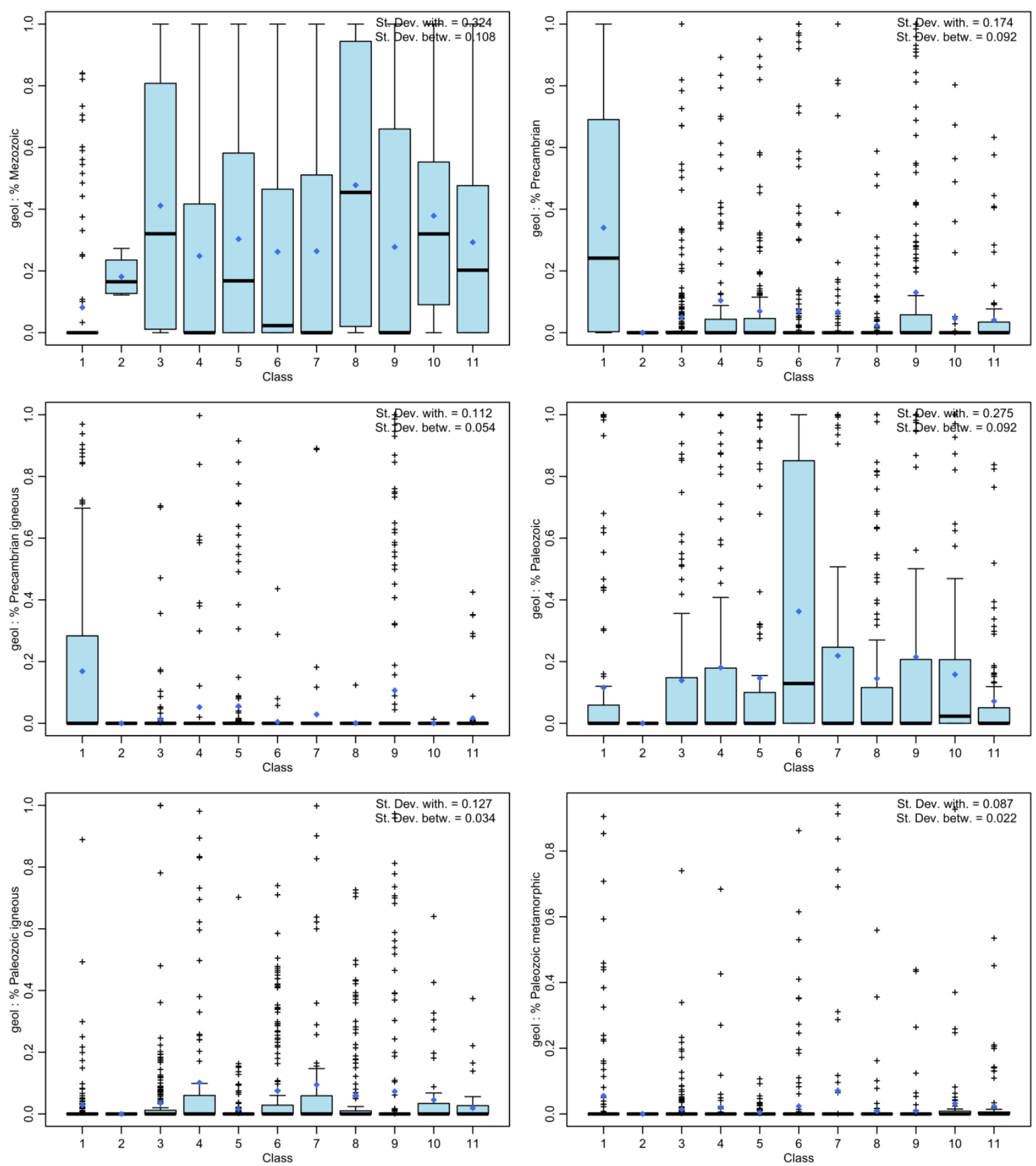

Figure X: boxplots of catchment descriptors in the different classes of the FS classification (6/7) 


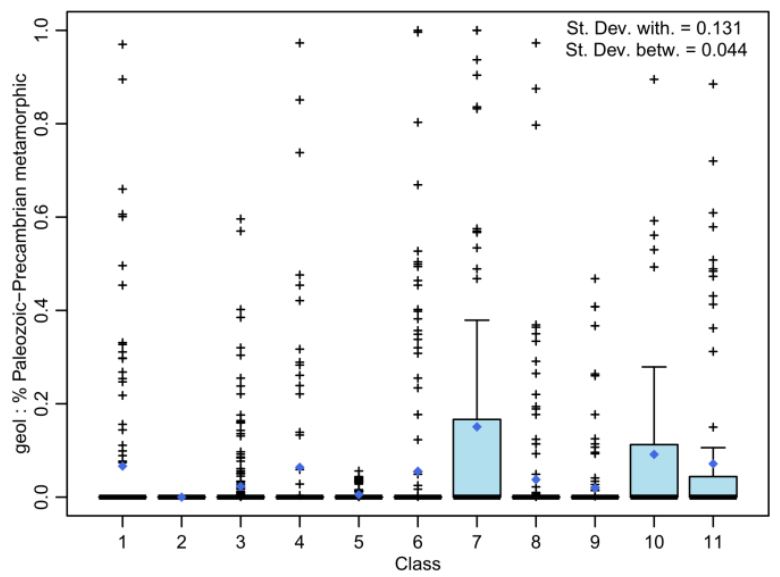

Figure Y: boxplots of catchment descriptors in the different classes of the FS classification $(7 / 7)$ 


\section{D.2 Classification based on catchment descriptors}

\section{D.2.1 Boxplots of flow signatures}
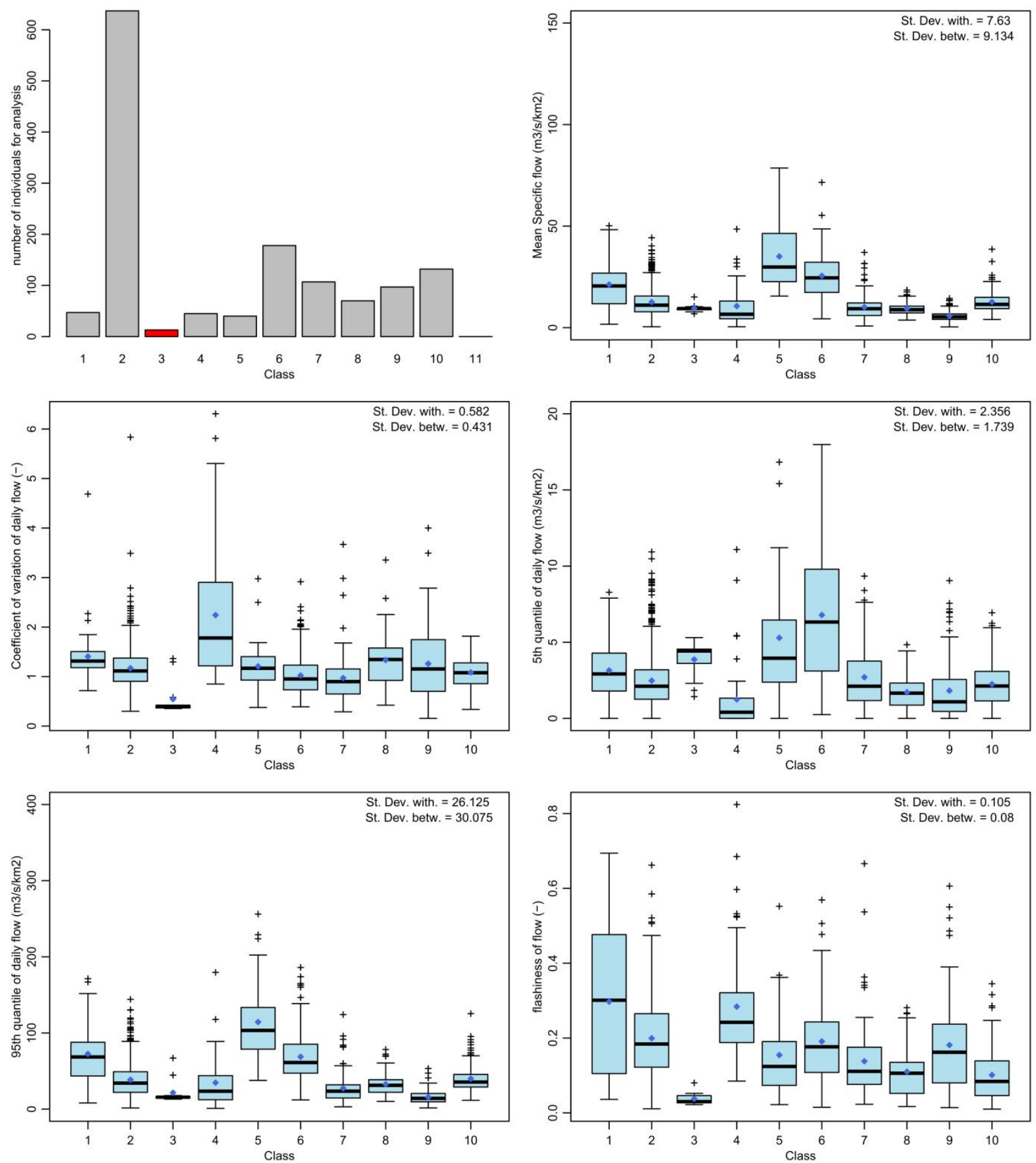

Figur Z: boxplots of flow signatures in the different classes of the CD classification (1/3) 

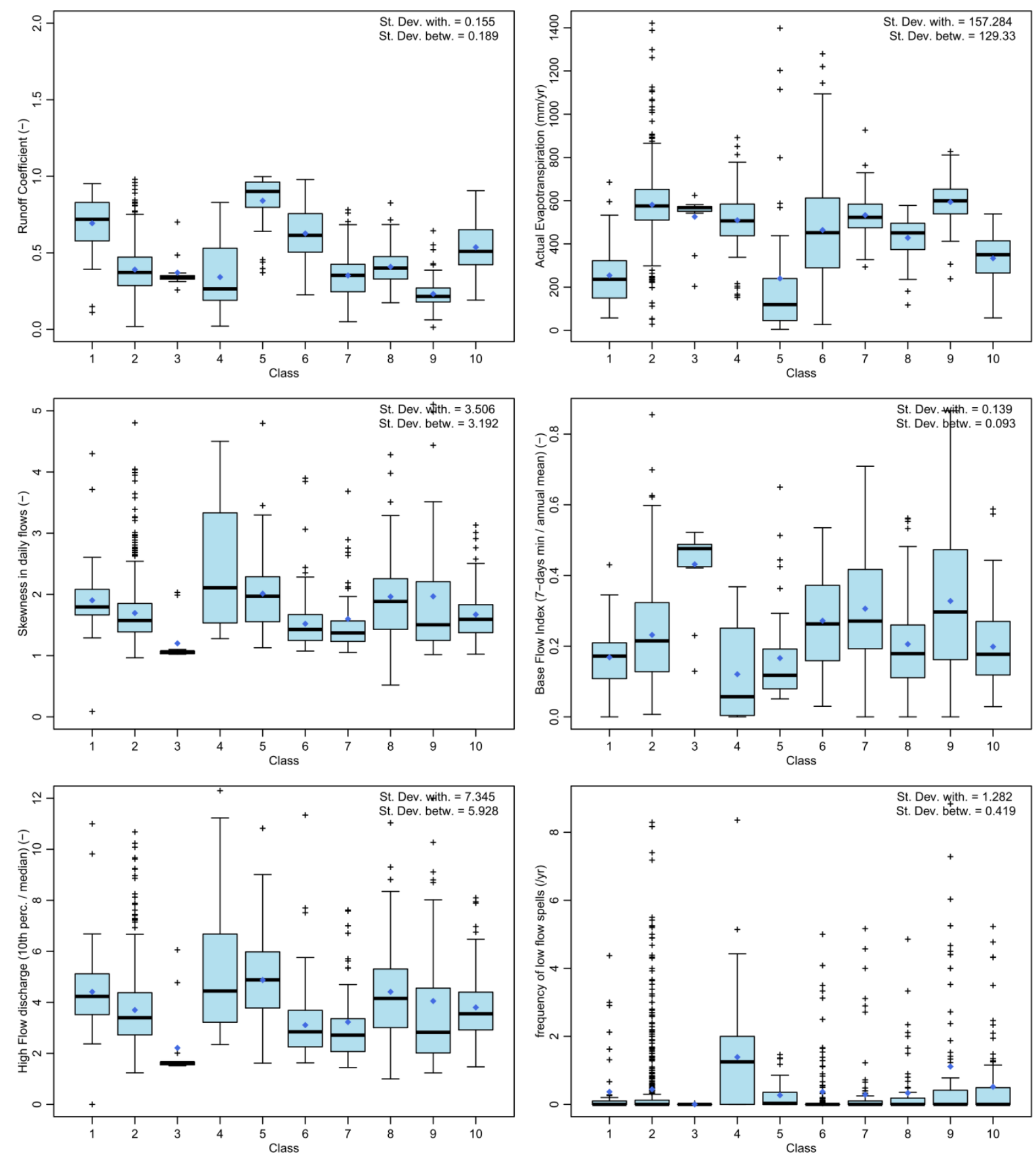

Figure AA: boxplots of flow signatures in the different classes of the $C D$ classification $(2 / 3)$ 

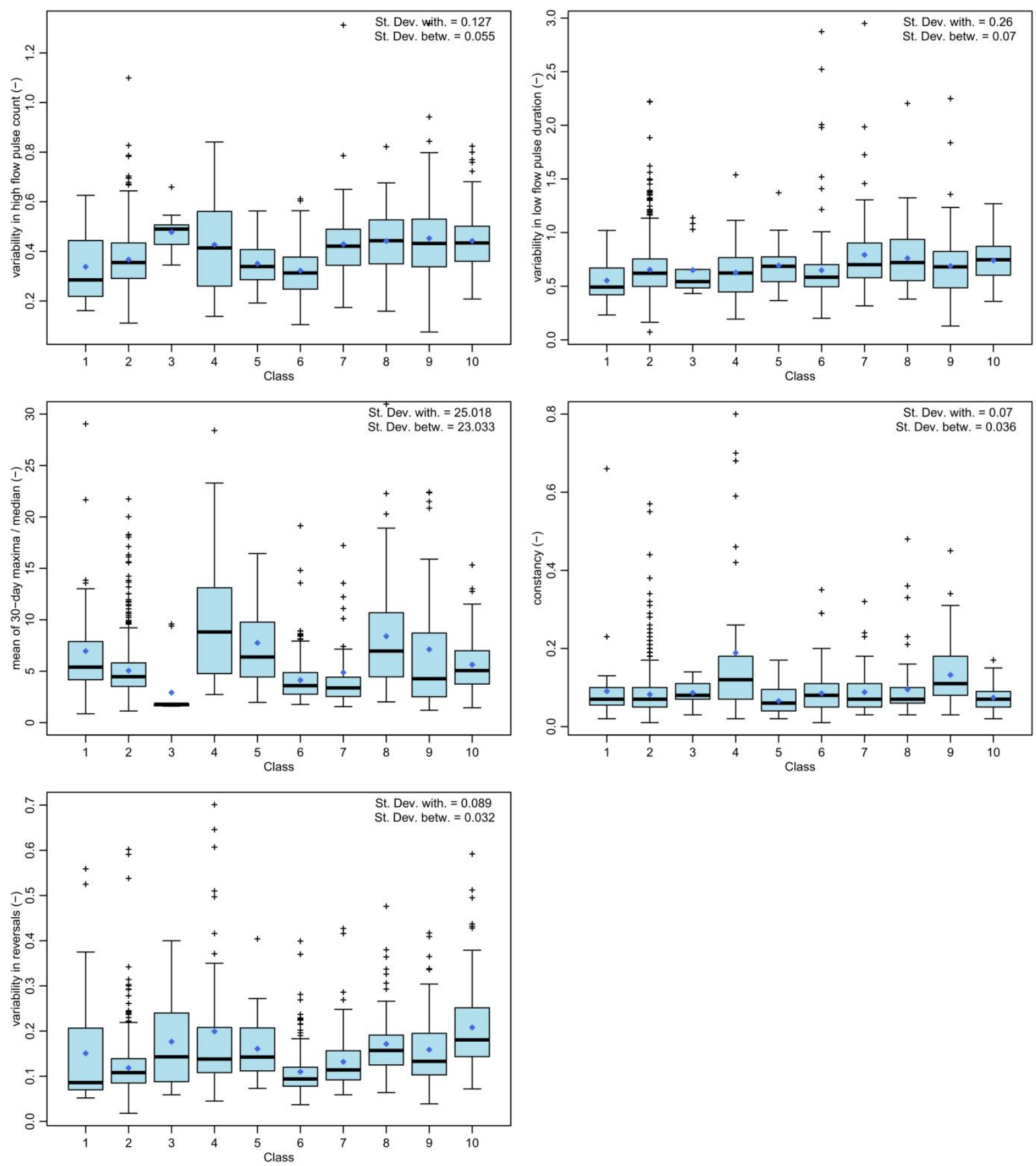

Figure BB: boxplots of flow signatures in the different classes of the CD classification $(3 / 3)$ 


\section{D.2.2 Boxplots of catchment descriptors}
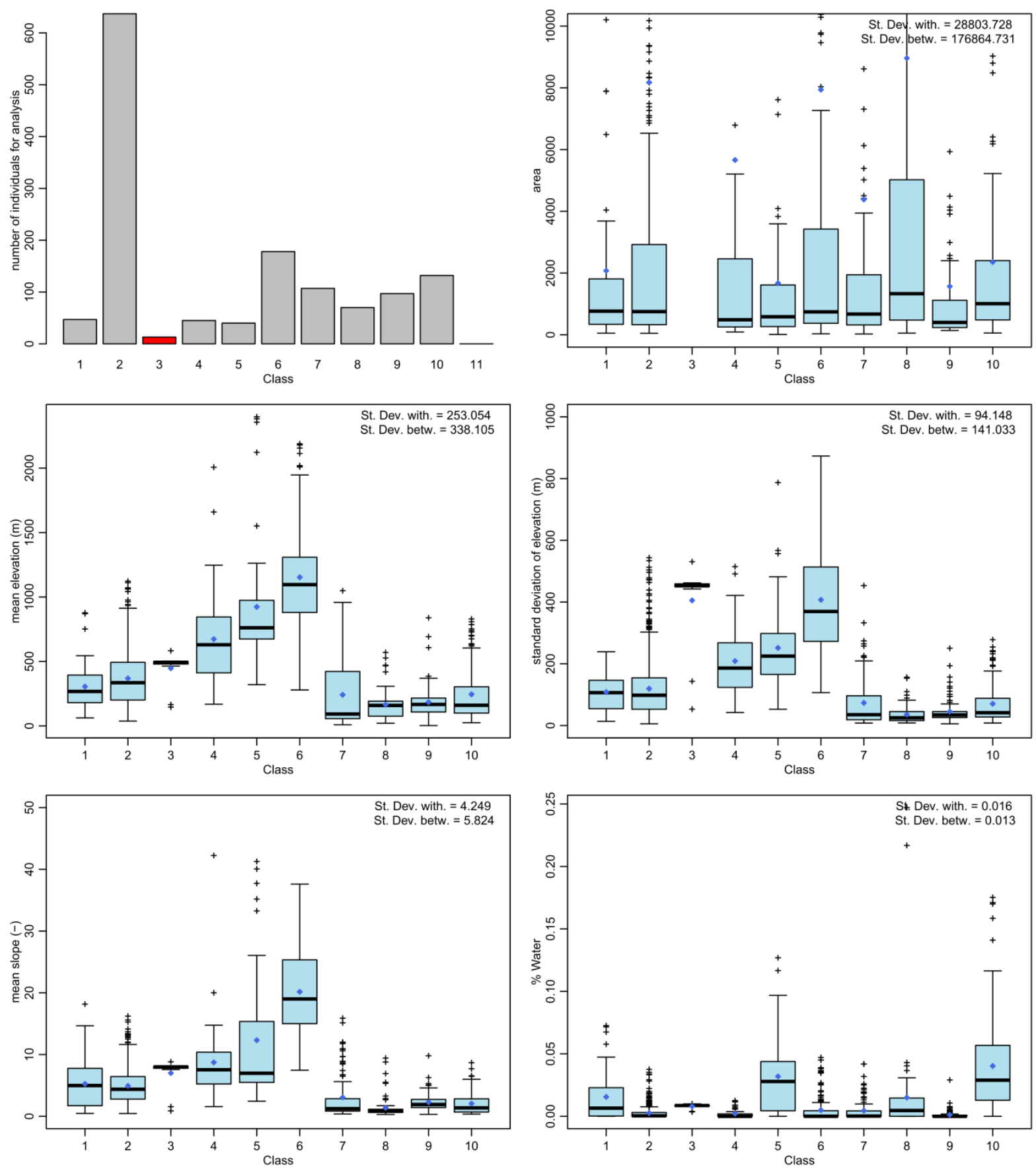

Figure CC: boxplots of catchment descriptors in the different classes of the CD classification (1/7). 

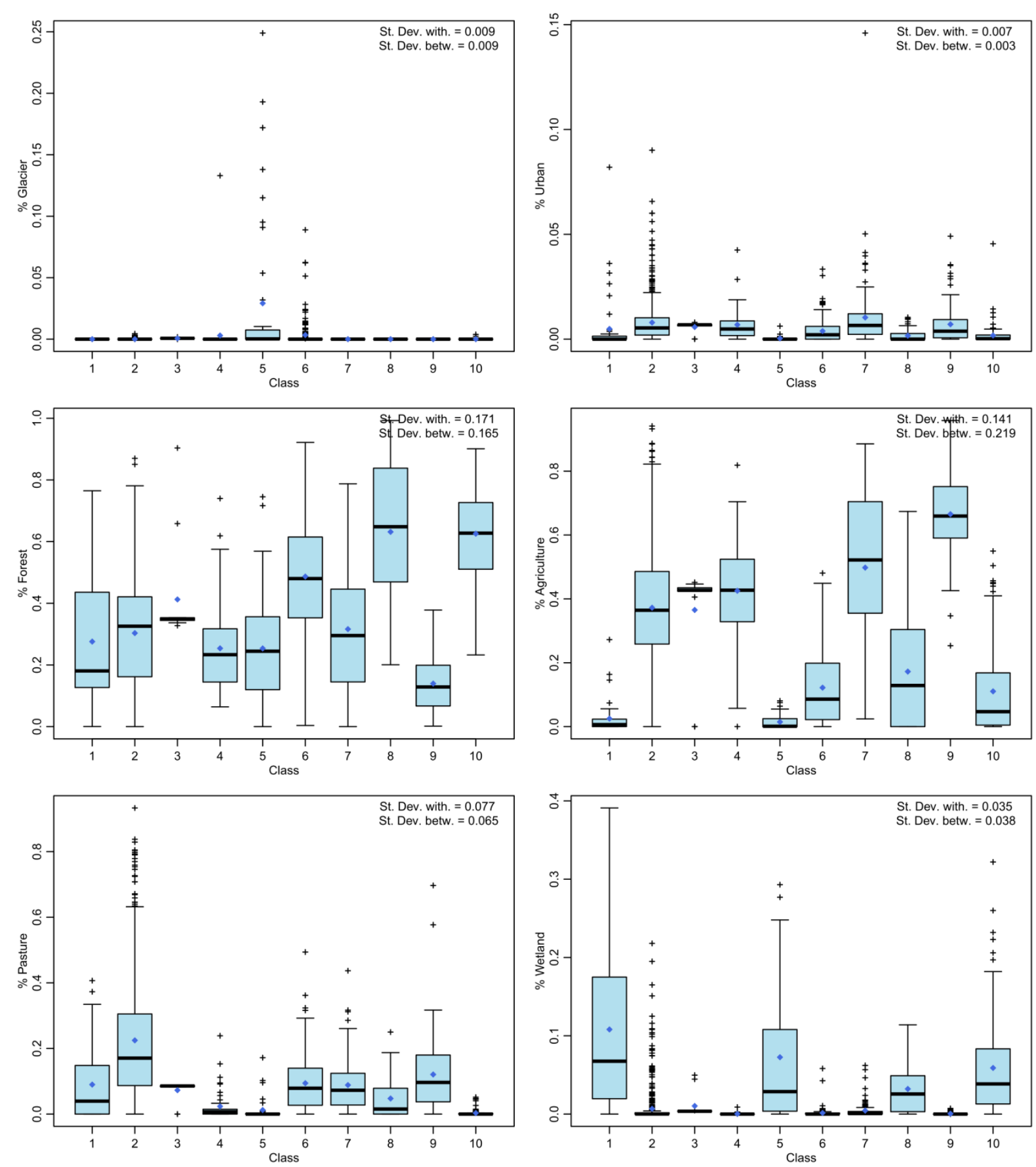

Figure DD: boxplots of catchment descriptors in the different classes of the CD classification (2/7). 

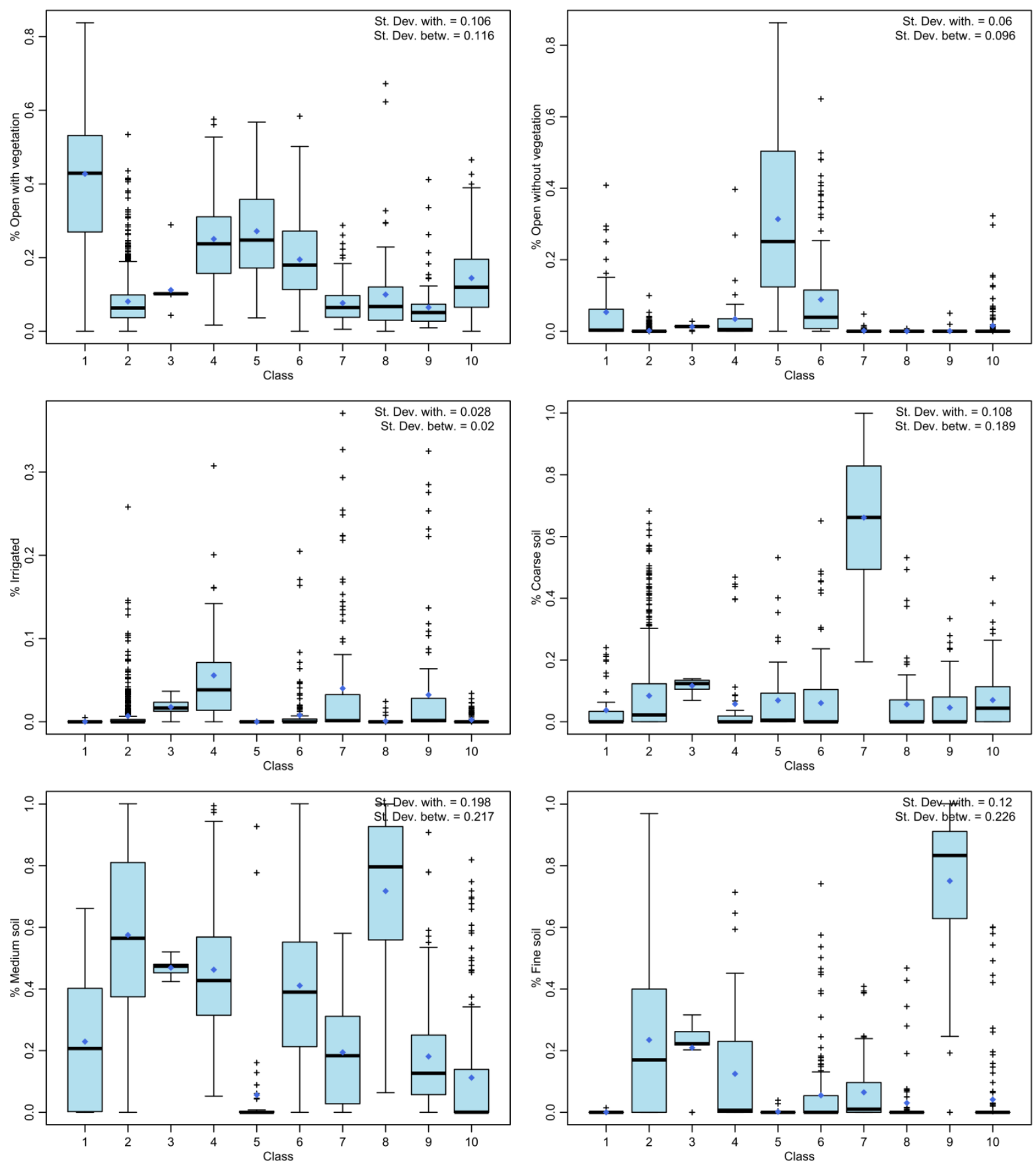

Figure EE: boxplots of catchment descriptors in the different classes of the CD classification (3/7). 

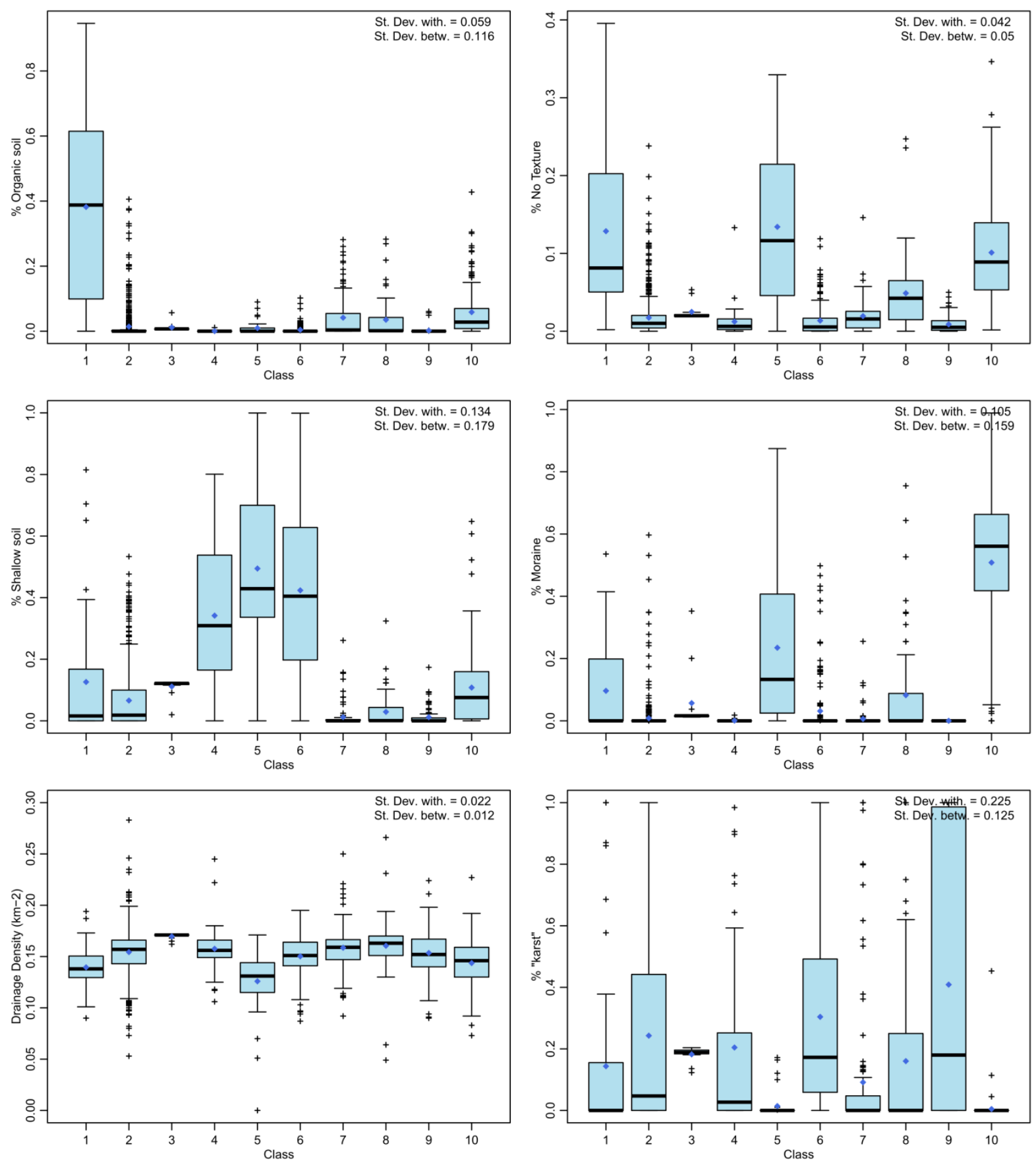

Figure FF: boxplots of catchment descriptors in the different classes of the CD classification (4/7). 

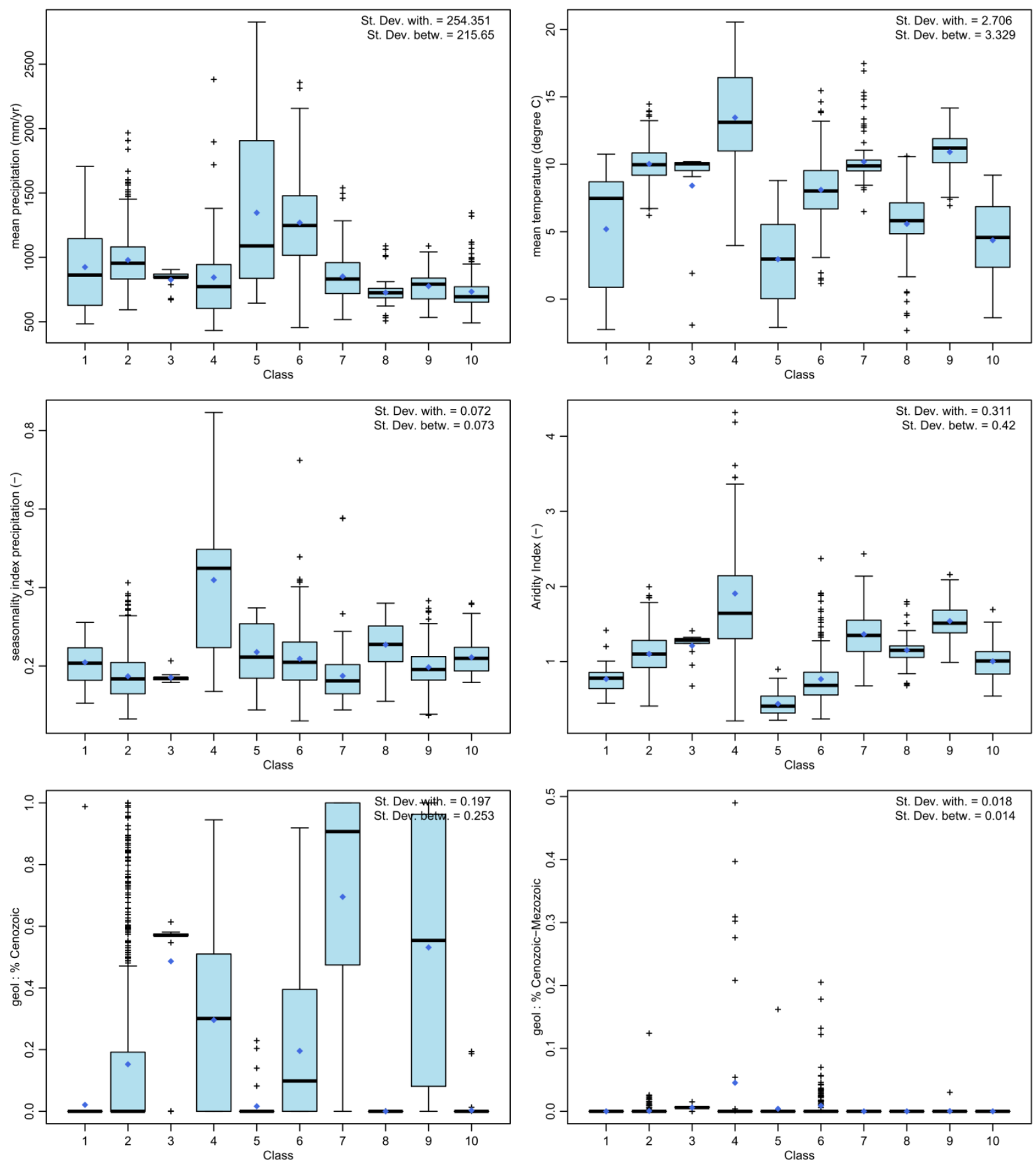

Figure GG: boxplots of catchment descriptors in the different classes of the CD classification (5/7). 

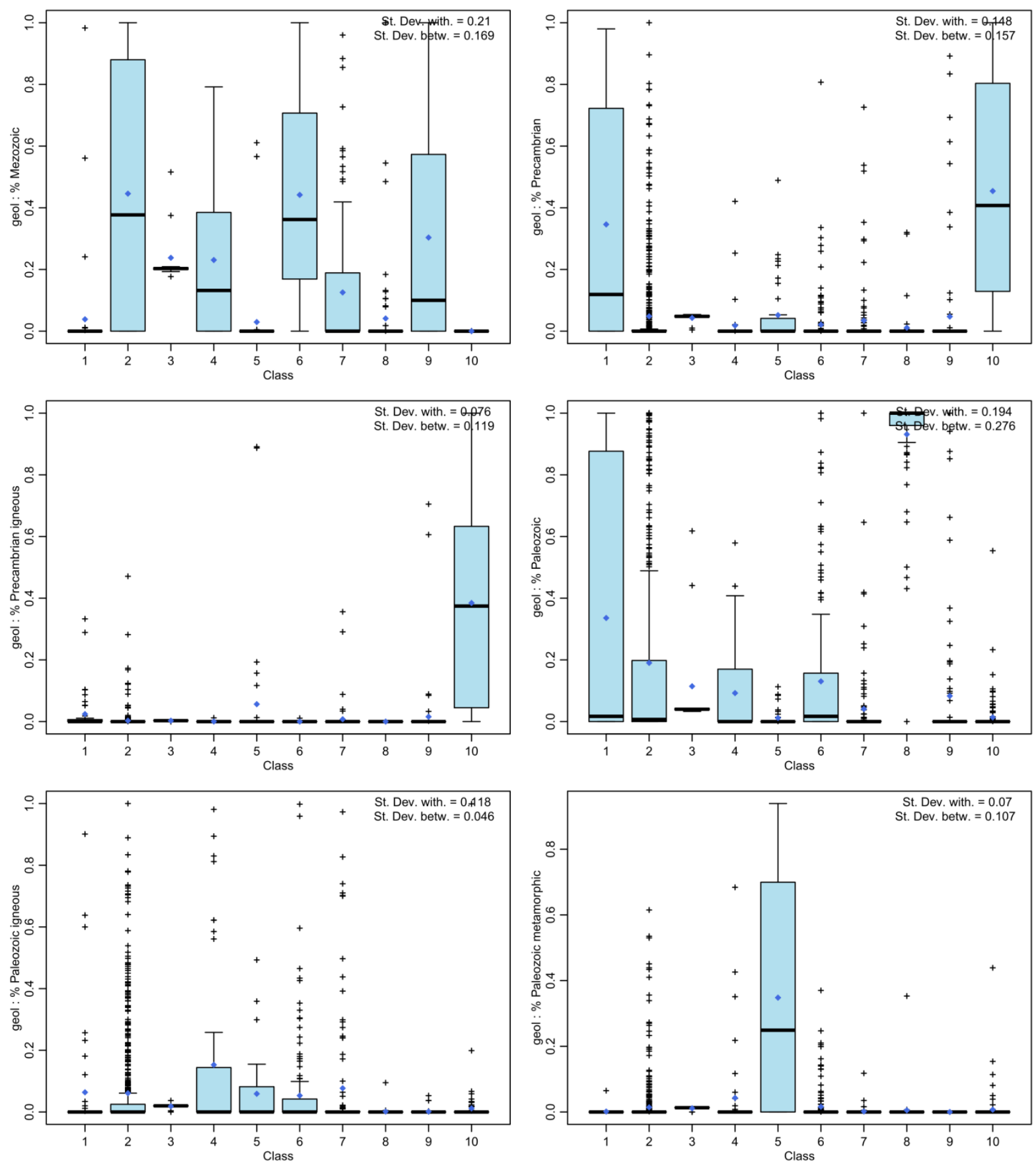

Figure HH: boxplots of catchment descriptors in the different classes of the CD classification (6/7). 


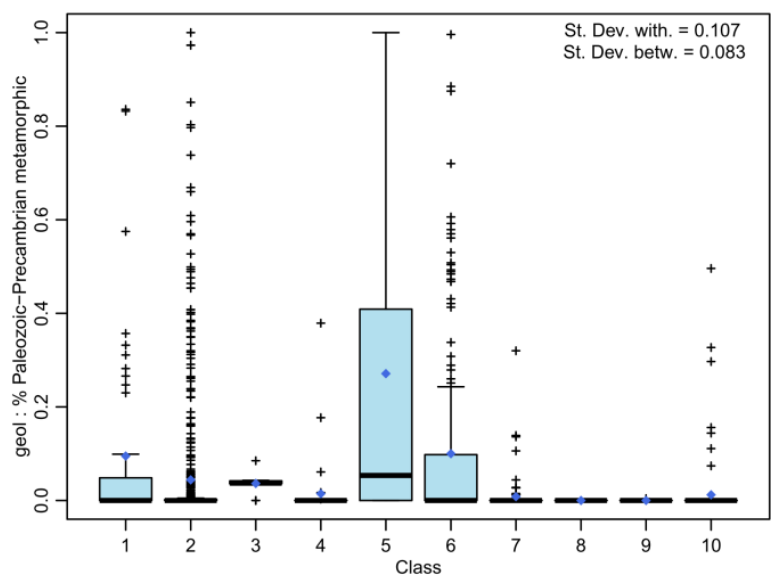

Figure II: boxplots of catchment descriptors in the different classes of the CD classification (7/7). 


\section{E. Linear regression coefficients}

\section{E.1 Linear regressions calibrated over the whole domain}

Table B: Coefficients of the linear regression calibrated over the whole domain. One model is calibrated for each flow signature and reads as a column in the table.

\begin{tabular}{|c|c|c|c|c|c|c|c|c|c|c|c|c|c|c|c|c|}
\hline & $\frac{0}{8}$ & ن & \% & ర్ & $\begin{array}{l}\frac{5}{\mathscr{D}} \\
\frac{\Phi}{4} \\
\underline{\omega} \\
\mathbb{\alpha}\end{array}$ & 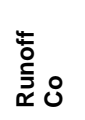 & 惡 & $\begin{array}{l}\frac{3}{d} \\
\frac{d}{\omega}\end{array}$ & $\overline{\bar{\alpha}}$ & $\frac{\underline{u}}{I}$ & 崩 & 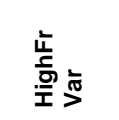 & 言 & 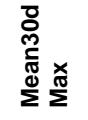 & $\begin{array}{l}\text { कू } \\
\overline{0} \\
0\end{array}$ & 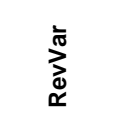 \\
\hline \begin{tabular}{|l|} 
Intercept) \\
area
\end{tabular} & -0.516 & $\begin{array}{c}-0.287 \\
-9.55 \mathrm{E}-07\end{array}$ & 0.823 & -19.4 & -0.038 & 0.643 & 16.3 & -15.2 & 0.282 & -40.2 & -0.582 & $\begin{array}{c}0.334 \\
1.47 \mathrm{E}-07\end{array}$ & 0.878 & -119 & -0.107 & 0.0345 \\
\hline meanElev & & & & & & & & & & & & & & & & \\
\hline stdElev & & -0.000518 & 0.00811 & -0.0265 & -0.000156 & & & & 0.000282 & & & & & & & \\
\hline meanSlope & & & & & & & & & & 0.144 & & & & & 0.00179 & \\
\hline Water & & -5.27 & 15.9 & & -1.11 & & & & 0.999 & & & 1.13 & & & & 0.516 \\
\hline \begin{tabular}{|l} 
Glacier \\
Urban
\end{tabular} & & & & & & & & & & & -12.4 & & -2.36 & & & \\
\hline Forest & & & & & & & & -5.51 & & & $-1<.4$ & & -2.36 & -33.7 & -0.0439 & \\
\hline Agriculture & -6.79 & & & -28.4 & & -0.199 & 214 & -7.28 & & & 0.894 & 0.0864 & & -52.9 & & \\
\hline Pasture & & & & & & & & & & 14.3 & & -0.0174 & -0.152 & & & \\
\hline Wetland & & & & 94.6 & 0.359 & 0.468 & & & & 26.2 & & & & & & 0.177 \\
\hline OpwithVeg & 10.5 & 0.676 & & 45.6 & 0.234 & 0.272 & -332 & & & 8.11 & & & -0.267 & & & \\
\hline OpwithoutVeg & 15.1 & & 3.03 & 52.6 & & 0.301 & -476 & -5.46 & & & & & & & & \\
\hline Irrigated & & & & & & & & & & & 3.6 & & & & & \\
\hline Coarse & & & 1.59 & & -0.096 & & & & & & & & & & & \\
\hline Medium & & & & & & & & & & & & & & & 0.017 & \\
\hline Fine & & & & & & & & & & & & & & & & \\
\hline \multicolumn{17}{|l|}{ Organic } \\
\hline \multirow{2}{*}{\multicolumn{17}{|c|}{$\begin{array}{l}\text { NoTexture } \\
\text { Shallow }\end{array}$}} \\
\hline Shallow & & & & & & 0.129 & & & -0.126 & & & & & -15.5 & -0.0292 & \\
\hline Moraine & & & & & & & & & & & & & & & & 3.50E-02 \\
\hline DrainDens & -27 & & & & & & 852 & & & & & & & & & \\
\hline Pmean & 0.0233 & 0.000574 & 0.00232 & 0.0693 & 0.000115 & & 0.264 & 0.00974 & $-4.64 \mathrm{E}-05$ & 0.0181 & & $-2.94 \mathrm{E}-05$ & -0.000146 & 0.0695 & $9.60 \mathrm{E}-05$ & $2.98 \mathrm{E}-05$ \\
\hline Tmean & -0.342 & -0.032 & & & 0.0134 & & 10.8 & -0.518 & -0.00501 & -1.14 & 0.089 & -0.012 & & -3.51 & -0.0101 & -0.00908 \\
\hline PSI & & 1.91 & & & & & & 11.8 & -0.233 & 23.7 & 2.01 & & & 86.5 & 0.0938 & 0.125 \\
\hline Al & & 0.858 & -1.43 & & & -0.185 & & 13.6 & & 26.5 & & 0.135 & & 98.2 & 0.16 & 0.113 \\
\hline $\mathrm{Cz}$ & & -0.402 & 0.624 & & & & & -2.34 & 0.169 & -5.38 & -0.96 & & & -16.8 & & -0.019 \\
\hline CzMz & & & & & & & & & & & & & & & & \\
\hline Mz & & & & & & & & & 0.0886 & & -0.528 & & & & & \\
\hline \multirow{2}{*}{\multicolumn{17}{|c|}{$\begin{array}{l}\mathrm{pCm} \\
\mathrm{pCmi}\end{array}$}} \\
\hline & & & & & & & & & & & & & & & & \\
\hline Pzi & & & -2.49 & & & & & & -0.0548 & & & & 0.167 & & & \\
\hline Pzm & & & -2.38 & & & & & & & & & & & & & \\
\hline PzpCmm & & & & & & & & & & & & & & & & \\
\hline
\end{tabular}




\section{E.2 Linear regressions calibrated inside each classification group}

\section{E.2.1 CART Classification}

Table C: Coefficients of the linear regression calibrated in group 1 of the CART classification. One model is calibrated for each flow signature and reads as a column in the table.

\begin{tabular}{|c|c|c|c|c|c|c|c|c|c|c|c|c|c|c|c|c|}
\hline & $\frac{0}{8}$ & गे & $\stackrel{8}{8}$ & \% & 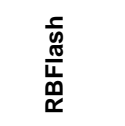 & 产 & 惡 & $\begin{array}{l}\frac{3}{0} \\
\frac{\nu}{\omega}\end{array}$ & $\overline{\underline{M}}$ & 은 & 峁 & 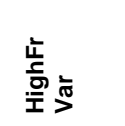 & لَّ & 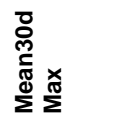 & 离 & 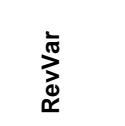 \\
\hline (Intercept) & 2.53 & 1.38 & $\begin{array}{l}-1.83 \\
\end{array}$ & 14.4 & |0.237 & 0.808 & \begin{tabular}{|l|}
-79.8 \\
\end{tabular} & 2.36 & 0.211 & -1.36 & (0.603 & $\begin{array}{l}0.216 \\
\end{array}$ & 0.699 & $\begin{array}{ll}9.88 \\
\end{array}$ & \begin{tabular}{l|l|}
0.0895 \\
\end{tabular} & 0.232 \\
\hline & & & & $-3.47 \mathrm{E}-05$ & $-3.70 \mathrm{E}-07$ & & & & & & & 1.71E-07 & & & & $5.27 \mathrm{E}-08$ \\
\hline meanElev & & & & & & & & & & & & & & & & $1.14 \mathrm{E}-05$ \\
\hline stdElev & & & 0.00884 & -0.00285 & & & & & 0.000265 & 0.00353 & & & & & $-1.12 \mathrm{E}-05$ & $-4.08 \mathrm{E}-05$ \\
\hline meanSlope & & -0.0114 & & & & & & -0.0116 & & & & & & -0.0806 & & \\
\hline Water & & -5.72 & 22.5 & 56.2 & -1.47 & & & -9.41 & 0.943 & 6.83 & & 1.76 & & -58.3 & & \\
\hline Glacier & & & 5.9 & & & & & & 0 & & & & & & & \\
\hline Urban & -66.9 & -4.37 & & & 0.657 & -1.79 & 2110 & 3.07 & & -63.7 & -5.72 & & & 37.2 & 0.294 & -1.17 \\
\hline \multirow{2}{*}{\multicolumn{17}{|c|}{$\begin{array}{l}\text { Forest } \\
\text { Agriculture }\end{array}$}} \\
\hline & & & & & -0.0749 & & & & & & & & & & & \\
\hline \multirow{2}{*}{\multicolumn{17}{|c|}{$\begin{array}{l}\text { Pasture } \\
\text { Wetland }\end{array}$}} \\
\hline & & & & & & & & & & & & & & & & \\
\hline \multirow{2}{*}{\multicolumn{17}{|c|}{$\begin{array}{l}\text { OpwithVeg } \\
\text { OpwithoutVeg }\end{array}$}} \\
\hline \multirow{2}{*}{\multicolumn{17}{|c|}{ Irrigated }} \\
\hline & & & & & & & & & & & & & & & & \\
\hline Coarse & & -0.495 & & & -0.0884 & & & -1.26 & 0.116 & & & & & -8.46 & & \\
\hline Medium & & & & & & & & & & & & . & & & & -0.0245 \\
\hline \multirow{2}{*}{\multicolumn{17}{|c|}{ Fine }} \\
\hline \multicolumn{15}{|l|}{ Organic } & & \\
\hline NoTexture & & & & & & & & & & & & & & & & 0.276 \\
\hline \multicolumn{17}{|l|}{ Shallow } \\
\hline Moraine & & -0.00813 & & & -0.127 & & & -0.409 & & 7.21 & & & & -1.53 & & \\
\hline \multicolumn{17}{|l|}{ DrainDens } \\
\hline Pmean & 0.0198 & & 0.00357 & 0.0542 & & $-2.14 \mathrm{E}-05$ & 0.375 & & & -0.00447 & $-8.04 \mathrm{E}-05$ & & & & & $-8.89 \mathrm{E}-05$ \\
\hline Tmean & & & & & & & & & & 1.03 & & & -0.00255 & & & \\
\hline \multicolumn{17}{|l|}{ PSI } \\
\hline Al & -6.66 & & & -22.4 & & -0.314 & 210 & & & & & 0.138 & & & & \\
\hline \multirow{2}{*}{\multicolumn{17}{|c|}{$\begin{array}{l}\text { Cz } \\
\text { CzMz }\end{array}$}} \\
\hline & & & & & & & & & & & & & & & & \\
\hline Mz & & & & & & & & & & & & & & & 0.000454 & \\
\hline \multicolumn{17}{|l|}{$\mathrm{pCm}$} \\
\hline \multicolumn{17}{|l|}{ pCmi } \\
\hline $\mathrm{Pz}$ & 1.1 & & & & & 0.0223 & -34.7 & & & & & & & & & \\
\hline \multicolumn{17}{|l|}{ Pzi } \\
\hline Pzm & 7.22 & & -1.83 & 37.5 & 0.00628 & 0.208 & -228 & 1.03 & -0.117 & 5.33 & 0.0231 & & & & & \\
\hline PzpCmm & 3.78 & 0.206 & -0.82 & 14.4 & & 0.102 & -119 & -0.467 & -0.0773 & 1.95 & & & & -2.92 & & \\
\hline
\end{tabular}




\begin{tabular}{|c|c|c|c|c|c|c|c|c|c|c|c|c|c|c|c|c|}
\hline & $\frac{0}{8}$ & गे & $\mathscr{8}$ & \% & 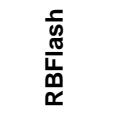 & 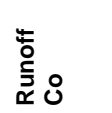 & $\stackrel{5}{\underline{4}}$ & $\begin{array}{l}\frac{3}{0} \\
\frac{\partial}{\omega}\end{array}$ & $\overline{\bar{u}}$ & $\frac{0}{\underline{1}}$ & 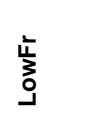 & 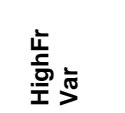 & 芌 & 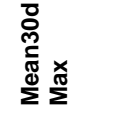 & 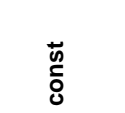 & 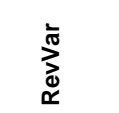 \\
\hline (Intercept) & 1.82 & 0.938 & 4.53 & 9.86 & |0.157 & 0.691 & 108 & 2.23 & 0.21 & 5.02 & \begin{tabular}{|c|}
-0.184 \\
\end{tabular} & 0.398 & 0.689 & $\begin{array}{l}9.53 \\
\end{array}$ & \begin{tabular}{l|l}
0.0422 \\
\end{tabular} & 0.0916 \\
\hline area & & $-1.52 \mathrm{E}-06$ & & $-2.97 \mathrm{E}-05$ & $-3.36 \mathrm{E}-07$ & & & & & & & $\begin{array}{l}1.54 \mathrm{E}-07 \\
\end{array}$ & & & & \\
\hline \multicolumn{17}{|l|}{ meanElev } \\
\hline stdElev & & & & & & & & -0.000644 & & -0.00223 & & & & -0.00575 & & \\
\hline meanSlope & 0.0911 & & 0.171 & & & & -2.57 & & & & & & & & & \\
\hline Water & & & & & -1.67 & & & & & & & 1.52 & 0.904 & & & \\
\hline Glacier & & & 18 & & & & -632 & & & & & & & & & \\
\hline Urban & & & & & & & & 3.54 & & -1.79 & & -0.825 & -2.02 & 34.4 & & -0.781 \\
\hline \multicolumn{17}{|l|}{ Forest } \\
\hline Agriculture & -8.51 & & & -29.1 & & -0.281 & 359 & & & & & 0.0644 & & & 0.0653 & \\
\hline Pasture & & 0.159 & -0.735 & & 0.176 & & & & -0.131 & & & & & & & \\
\hline \multirow{2}{*}{\multicolumn{17}{|c|}{ Wetland }} \\
\hline & & & & & 0.198 & & & & & & & & & & & \\
\hline \multicolumn{17}{|l|}{ OpwithoutVeg } \\
\hline Irrigated & & & & & & & & 11.3 & & 23.6 & & & & 69.4 & & \\
\hline Coarse & 1.88 & -0.292 & 2.22 & 1.58 & -0.0653 & 0.0701 & -50.2 & -1.39 & & -3.07 & & & & -11.3 & & \\
\hline \multicolumn{17}{|l|}{ Medium } \\
\hline Fine & & & 0.342 & & & & & & & & & & & & & -0.00113 \\
\hline Organic & & & & & & & & & & & & & & & 0.0113 & \\
\hline NoTexture & & -1.03 & & & & & & & & & & & & & & \\
\hline Shallow & 7.78 & & & 26.7 & & 0.172 & -226 & & -0.0725 & & & & & & & 0.016 \\
\hline \multirow{2}{*}{\multicolumn{17}{|c|}{$\begin{array}{l}\text { Moraine } \\
\text { DrainDens }\end{array}$}} \\
\hline & & & & & & & & & & & & & & & & \\
\hline Pmean & 0.0181 & -0.000246 & & 0.052 & & & 0.35 & & & & & -0.000114 & & & $-2.39 \mathrm{E}-05$ & $2.76 \mathrm{E}-05$ \\
\hline Tmean & & & & & & & & & & & & & & & & -0.0133 \\
\hline PSI & & 2.69 & & & & & & & & & & & & & 0.247 & \\
\hline Al & -3.55 & & -2.49 & -9.5 & & -0.176 & & & & & 0.614 & 0.0609 & & & & 0.126 \\
\hline $\mathrm{Cz}$ & -0.432 & & & -6.23 & & -0.0241 & 21.5 & -0.444 & 0.159 & -1.37 & & & & & & \\
\hline CzMz & & & & & 0.224 & & & & & & & & & & & \\
\hline $\mathrm{Mz}$ & & & & & & & & & 0.078 & & & & & & & \\
\hline \multirow{2}{*}{\multicolumn{17}{|c|}{$\begin{array}{l}\mathrm{pCm} \\
\mathrm{pC} \mathrm{Cmi}\end{array}$}} \\
\hline & & & & & & & & & & & & & & & & \\
\hline \multicolumn{17}{|c|}{$-0.06 \varepsilon$} \\
\hline Pzi & & & -2.46 & & & & & & & & & & 0.0905 & & & \\
\hline \multicolumn{17}{|l|}{ Pzm } \\
\hline PzpCmm & & & & & & & & & & & & & & & & 0.0174 \\
\hline
\end{tabular}




\begin{tabular}{|c|c|c|c|c|c|c|c|c|c|c|c|c|c|c|c|c|}
\hline & $\frac{0}{0}$ & गे & 40 & \%ั & 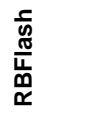 & ○ & $\stackrel{5}{\underline{4}}$ & $\begin{array}{l}\frac{3}{0} \\
\frac{\partial}{\omega}\end{array}$ & $\overline{\bar{n}}$ & $\frac{a}{\underline{x}}$ & 訔 & 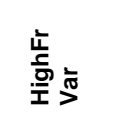 & 芌 & 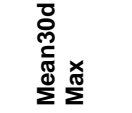 & $\begin{array}{l}\overline{0} \\
\overline{0} \\
\dot{0}\end{array}$ & 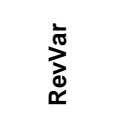 \\
\hline (Intercept) & -10.8 & 0.695 & -0.867 & \begin{tabular}{|l|}
-32.7 \\
\end{tabular} & 0.0648 & 0.688 & 342 & -18.6 & 0.279 & -34.2 & 0.738 & 0.258 & 0.691 & & $\begin{array}{l}-0.0402 \\
\end{array}$ & 0.095 \\
\hline area & & $-1.63 \mathrm{E}-06$ & & & & & & $-7.31 \mathrm{E}-07$ & & $-2.81 E-06$ & & & & $-2.90 \mathrm{E}-06$ & $-3.61 \mathrm{E}-08$ & $5.68 \mathrm{E}-08$ \\
\hline meanElev & & & & & & & & & & & & & & & & \\
\hline stdElev & & & & & & & & & & & & $-4.69 \mathrm{E}-05$ & & & & $-4.84 \mathrm{E}-05$ \\
\hline meanSlope & 0.23 & & & 0.654 & & 0.00191 & -7.25 & & & & & & & & & \\
\hline Water & & -4.24 & & & & & & & & & & & & & & \\
\hline \multicolumn{17}{|l|}{ Glacier } \\
\hline Urban & & & & & & & & & 2.15 & & & & & & & \\
\hline Forest & & & 0.738 & -6.72 & & & & & & & -0.704 & & & & & \\
\hline \multirow{2}{*}{\multicolumn{17}{|c|}{$\begin{array}{l}\text { Agriculture } \\
\text { Pasture }\end{array}$}} \\
\hline & & & & & 0.121 & & & 6.51 & & 12.7 & & & & 47.3 & -0.0458 & \\
\hline \multirow{2}{*}{\multicolumn{17}{|c|}{$\begin{array}{l}\text { Wetland } \\
\text { OpwithVeg }\end{array}$}} \\
\hline & & & & & & & & & & & & & & & & \\
\hline OpwithoutVeg & 20 & & 5.97 & & & 0.309 & -632 & & & & & & & & & \\
\hline \multicolumn{17}{|l|}{ Irrigated } \\
\hline Coarse & & & & & -0.0931 & & & & & & & 0.00742 & & & & \\
\hline Medium & & 0.133 & & & & & & & & & & & & & & \\
\hline \multicolumn{17}{|l|}{ Fine } \\
\hline \multirow{2}{*}{\multicolumn{17}{|c|}{$\mid \begin{array}{l}\text { Organic } \\
\text { NoTexture }\end{array}$}} \\
\hline & & & & & & & & & & & & & & & & \\
\hline \multicolumn{17}{|l|}{ Shallow } \\
\hline Moraine & 10.7 & & & 43.6 & & & -336 & & & & & & & & & \\
\hline \multicolumn{17}{|l|}{ DrainDens } \\
\hline Pmean & 0.0233 & & 0.00502 & 0.0732 & & & 0.265 & 0.00946 & & 0.0175 & & & & 0.0689 & 3.91E-05 & \\
\hline Tmean & & -0.0164 & -0.192 & & 0.0119 & & & -0.669 & -0.00756 & -1.26 & & & & -5.08 & & \\
\hline PSI & & & & & & & & 16.1 & & 31.2 & & & & 115 & & \\
\hline Al & & 0.65 & & & & -0.264 & & 12.1 & & 22.5 & & 0.124 & & 89.1 & 0.0899 & 0.0455 \\
\hline Cz & & -0.445 & 1.67 & & & & & & 0.164 & & & -0.0137 & & & & \\
\hline CzMz & & 0.895 & & & 0.23 & -0.183 & & -3.2 & & -7.47 & -0.943 & -0.204 & & -23.7 & 0.158 & -0.101 \\
\hline \multirow{2}{*}{\multicolumn{17}{|c|}{$\begin{array}{l}\mathrm{Mz} \\
\mathrm{pCm}\end{array}$}} \\
\hline $\mathrm{pCm}$ & & & & & & & & & & & & & & & & \\
\hline \multirow{2}{*}{\multicolumn{17}{|c|}{$\begin{array}{l}\mathrm{pCmi} \\
\mathrm{Pz}\end{array}$}} \\
\hline $\mathbf{P z}$ & & & & & & & & & & & 0.0599 & & & & & \\
\hline Pzi & 0.532 & & & 7.12 & & & -16.8 & & & & & & & & & \\
\hline Pzm & & & & & 0.0501 & & & & & & & -0.00251 & & & & \\
\hline PzpCmm & & & & & 0.0786 & & & & & & & & & & & \\
\hline
\end{tabular}




\begin{tabular}{|c|c|c|c|c|c|c|c|c|c|c|c|c|c|c|c|c|}
\hline & $\frac{0}{0}$ & गे & 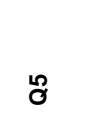 & \%ั & 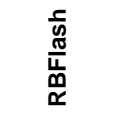 & 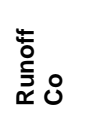 & $\stackrel{5}{\underline{4}}$ & $\begin{array}{l}\frac{3}{0} \\
\frac{\partial}{\omega}\end{array}$ & $\frac{\bar{n}}{\infty}$ & $\frac{0}{\underline{1}}$ & $\begin{array}{l}\frac{\pi}{3} \\
0 \\
0\end{array}$ & 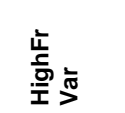 & 芌 & 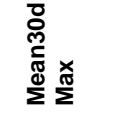 & $\begin{array}{l}\bar{m} \\
\overline{0} \\
\overline{0}\end{array}$ & 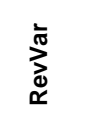 \\
\hline $\begin{array}{l}\text { (Intercept) } \\
\end{array}$ & -5.17 & 0.668 & -0.0717 & $\begin{array}{l}86.7 \\
\end{array}$ & 0.14 & 0.803 & 240 & -2.9 & $\begin{array}{ll}0.116 \\
\end{array}$ & \begin{tabular}{l|l}
-9.5 \\
\end{tabular} & \begin{tabular}{l|l|}
-0.652 \\
\end{tabular} & 0.592 & \begin{tabular}{l|l}
0.679 \\
\end{tabular} & -30.6 & 0.0867 & 0.195 \\
\hline area & & $-1.48 \mathrm{E}-06$ & & & $-3.52 \mathrm{E}-07$ & & & $-5.90 \mathrm{E}-08$ & 4.83E-07 & $-8.65 \mathrm{E}-06$ & & & & $1.71 \mathrm{E}-07$ & & \\
\hline meanElev & & & & & & & & & $9.00 \mathrm{E}-05$ & & & & & & & \\
\hline \multirow{2}{*}{\multicolumn{17}{|c|}{\begin{tabular}{|l} 
stdElev \\
meanSlope
\end{tabular}}} \\
\hline & & & & & 0.00263 & & & & & & & & & & & \\
\hline \multicolumn{17}{|l|}{ Water } \\
\hline Glacier & & & & & -0.762 & & & & & 53.5 & & & & & & -0.544 \\
\hline \multicolumn{17}{|l|}{ Urban } \\
\hline Forest & & & & & & & & & 0.127 & & & -0.0499 & & & & \\
\hline Agriculture & & & 0.604 & & & & & & 0.303 & & & & & & & \\
\hline Pasture & & & & & 0.194 & & & & & & & -0.176 & & & & \\
\hline Wetland & 21.5 & & & & & & & & & & & & & & & \\
\hline OpwithVeg & 17.1 & & & 54.2 & & & -518 & & & 15.5 & 0.537 & & & & 0.0246 & \\
\hline OpwithoutVeg & & & & & & & & & & & & & -0.0617 & & & \\
\hline \multicolumn{17}{|l|}{ Irrigated } \\
\hline Coarse & & & & & & & & 0.319 & & -3.09 & & 0.0189 & & & & \\
\hline Medium & & & -0.468 & & & & & & & 2.15 & & & & 8.29 & & -0.0305 \\
\hline \multirow{2}{*}{\multicolumn{17}{|c|}{$\begin{array}{l}\text { Fine } \\
\text { Organic }\end{array}$}} \\
\hline & & & & & & & & & & & & & & & & \\
\hline NoTexture & & -0.926 & & 1.33 & & & & -4.38 & 0.329 & & & & & & & \\
\hline Shallow & & -0.176 & 1.87 & & & & & & -0.0892 & & & & & & & \\
\hline \multicolumn{17}{|l|}{ Moraine } \\
\hline DrainDens & & & -0.13 & & & & & & & -3.8 & -1.03 & & & & & \\
\hline Pmean & 0.0225 & & 0.00502 & & & & 0.149 & & & & & -0.000174 & & & & \\
\hline Tmean & & & -0.219 & & & & 22 & & & & 0.0921 & & & & & -0.00487 \\
\hline PSI & & 2.89 & & & & & & 25.1 & & & & & & 181 & & \\
\hline AI & -4.38 & & & -46.4 & & -0.328 & & & -0.0572 & 10.8 & 0.314 & & & & & \\
\hline \multirow{2}{*}{\multicolumn{17}{|c|}{$\begin{array}{l}\mathrm{Cz} \\
\mathrm{Cz} \\
\end{array}$}} \\
\hline & & & & & & & & & & & & & & & & \\
\hline \multirow{2}{*}{\multicolumn{17}{|c|}{$\mathrm{Mz}$}} \\
\hline \multicolumn{16}{|l|}{$\mathrm{pCm}$} & \\
\hline pCmi & 3.07 & & & -9.16 & & 0.0537 & -34.3 & & & & 0.968 & & & & & \\
\hline $\mathrm{Pz}$ & & & & & 0.0112 & & & & & & 0.194 & & & 8.96 & & \\
\hline \multirow{2}{*}{\multicolumn{17}{|c|}{\begin{tabular}{|l} 
Pzi \\
Pzm
\end{tabular}}} \\
\hline & & & & & & & & & & & & & & & & \\
\hline PzpCmm & & & & 17.9 & & & & & & & & & & & & \\
\hline
\end{tabular}


Table G: Coefficients of the linear regression calibrated in group 6 of the CART classification. One model is calibrated for each flow signature and reads as a column in the table.

\begin{tabular}{|c|c|c|c|c|c|c|c|c|c|c|c|c|c|c|c|c|}
\hline & के & गे & $\stackrel{8}{8}$ & \%ั & 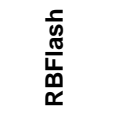 & 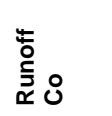 & $\stackrel{\square}{\underline{0}}$ & $\begin{array}{l}\frac{3}{0} \\
\text { के }\end{array}$ & $\frac{\bar{u}}{\infty}$ & $\frac{0}{\underline{\underline{u}}}$ & 崩 & 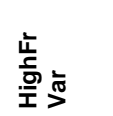 & 言 & 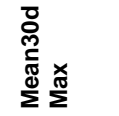 & 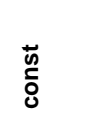 & 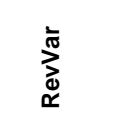 \\
\hline (Intercept) & 0.0645 & -0.225 & 4.63 & -6.79 & 0.0924 & o.555 & 153 & -17.4 & 0.265 & -25.1 & 0.556 & 0.225 & 0.826 & -17.6 & \begin{tabular}{|l|}
0.0917 \\
\end{tabular} & 0.126 \\
\hline area & & & $-2.64 \mathrm{E}-06$ & & & & & & & & & & & & & \\
\hline meanElev & & & -0.0016 & -0.00674 & $-4.84 E-05$ & & & & & & & & & & & \\
\hline stdElev & & -0.00138 & 0.00899 & & & & & $6.98 \mathrm{E}-05$ & 0.000347 & -0.000427 & & & & 0.00292 & & $-8.91 \mathrm{E}-05$ \\
\hline meanSlope & & 0.0198 & 0.0978 & & & & & & & & & & & & & \\
\hline Water & & & & & -1.89 & & & 1.39 & & & & 1.77 & & -105 & & 1 \\
\hline \multirow{2}{*}{\multicolumn{17}{|c|}{ Glacier }} \\
\hline & & & & & & & & & & & & & & & & \\
\hline Forest & & & & & & & & & & & & & & & -0.0185 & 0.0418 \\
\hline Agriculture & -7.77 & & & -39.2 & & -0.494 & 329 & & & & & & & & & \\
\hline Pasture & & & & & & & & & & & & & & 5.08 & & \\
\hline Wetland & & & & & & & & & & & & & & & -0.0841 & \\
\hline OpwithVeg & & 1.17 & & & 0.158 & 0.353 & & 7.51 & & 17.3 & & & & & & \\
\hline OpwithoutVeg & & & & & & & & & & & & & & & & \\
\hline Irrigated & & -0.966 & & & & & & -9.93 & & & & & & 9.14 & & 0.148 \\
\hline \multirow{2}{*}{\multicolumn{17}{|c|}{\begin{tabular}{|l} 
Coarse \\
Medium
\end{tabular}}} \\
\hline & & & & & & & & & & & & & & & & \\
\hline \multicolumn{17}{|l|}{ Fine } \\
\hline Organic & 11.6 & & & 41.6 & 0.11 & & -382 & & -0.0776 & 0.658 & & -0.00708 & & & & \\
\hline \multicolumn{17}{|l|}{ NoTexture } \\
\hline Shallow & 10.1 & & & 33.9 & & & -302 & & -0.155 & & & & & & & \\
\hline \multicolumn{17}{|l|}{ Moraine } \\
\hline \multicolumn{17}{|l|}{ DrainDens } \\
\hline Pmean & 0.0193 & 0.000464 & & 0.0618 & 0.000112 & & 0.315 & 0.00946 & $-6.89 \mathrm{E}-05$ & 0.0105 & $-6.81 \mathrm{E}-05$ & & -0.000167 & -0.0113 & & \\
\hline Tmean & & & & & & & & -0.444 & & & & & & & & \\
\hline PSI & & & -1.38 & & & & & & & & & -0.0517 & & 179 & & \\
\hline Al & -3.19 & 0.81 & -2.55 & & & & & 12.2 & & 15.6 & & 0.144 & & & & \\
\hline $\mathrm{Cz}$ & & & 1.27 & & -0.0224 & & & & & & & -0.0102 & 0.0174 & & 0.0307 & \\
\hline CzMz & & & & & & & & & & & & & & & & \\
\hline \multicolumn{17}{|l|}{$\mathrm{Mz}$} \\
\hline \multirow{2}{*}{\multicolumn{17}{|c|}{$\begin{array}{l}\mathrm{pCm} \\
\mathrm{pCCmi}\end{array}$}} \\
\hline & & & & & & & & & & & & & & & & \\
\hline \multicolumn{17}{|c|}{0.00396} \\
\hline Pzi & & & & & & & & & & & & -0.0173 & 0.147 & & & \\
\hline Pzm & & 0.46 & & & & & & & & & & & & & & \\
\hline PzpCmm & & & & & & & & & & & & & & & & \\
\hline
\end{tabular}




\begin{tabular}{|c|c|c|c|c|c|c|c|c|c|c|c|c|c|c|c|c|}
\hline & $\frac{0}{8}$ & ơ & $\stackrel{4}{0}$ & ஜூ & 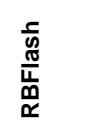 & 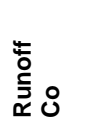 & 莸 & $\begin{array}{l}\frac{3}{0} \\
\frac{0}{\omega}\end{array}$ & $\underset{\mathbf{m}}{\overline{\mathbf{u}}}$ & $\frac{\underline{Q}}{\underline{x}}$ & 崩 & 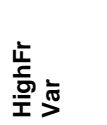 & 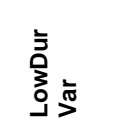 & 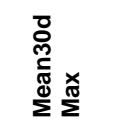 & $\begin{array}{l}\overline{\mathscr{\omega}} \\
\overline{0}\end{array}$ & 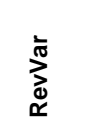 \\
\hline 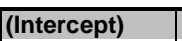 & 33.6 & 1.25 & 2.77 & \begin{tabular}{l|l|}
99.4 \\
\end{tabular} & 0.118 & 0.505 & 106 & -3.37 & 0.275 & 5.37 & -1.96 & 0.383 & |0.663 & -24.2 & \begin{tabular}{l|}
0.0876 \\
\end{tabular} & 0.224 \\
\hline area & & $-1.69 \mathrm{E}-06$ & $3.71 \mathrm{E}-06$ & $-3.76 \mathrm{E}-05$ & & & & $-3.83 \mathrm{E}-07$ & $4.52 \mathrm{E}-07$ & $-7.73 \mathrm{E}-06$ & & & & $-1.54 \mathrm{E}-05$ & $-1.04 \mathrm{E}-08$ & \\
\hline meanElev & & & & & & & & & & & 0.000112 & & $-1.64 \mathrm{E}-05$ & & & \\
\hline stdElev & & & & & & & & & & & -0.0012 & & & & & \\
\hline meanSlope & & -0.00845 & & & & & & & & & & & & & & -0.00248 \\
\hline Water & -10.7 & -5.28 & & & & & & & & & & & & & & \\
\hline Glacier & & & & & -0.404 & & & & & & & & & & & \\
\hline Urban & & & & & & -3.72 & & & & & & & & & & \\
\hline Forest & & & & -7.2 & & & & & & & & & & & & \\
\hline Agriculture & & & & & & & 448 & & & & & & 0.0756 & & & 0.00801 \\
\hline Pasture & & & & & 0.223 & & & & & & & -0.257 & & & & \\
\hline Wetland & & 0.537 & & 26.6 & -0.037 & & & -3.73 & & & & & -0.168 & & & \\
\hline OpwithVeg & & & & & & & & & & & & & & & 0.0311 & \\
\hline OpwithoutVeg & & & & & 0.0699 & & & & & & & & & & & \\
\hline Irrigated & & & & & & & & & & & & & & & & \\
\hline Coarse & & -0.502 & & & & & 1.2 & -0.105 & & -2.91 & & & & -12.6 & & \\
\hline Medium & & & & & & & & & & & & 0.0379 & & & & -0.0409 \\
\hline Fine & & & & & 0.00241 & & & & & & & 0.084 & & & & -0.0204 \\
\hline Organic & & & & & 0.169 & & & & & & & -0.0126 & & & & -0.071 \\
\hline NoTexture & & & & & & & & & & & & 0.19 & & & & \\
\hline Shallow & & & & & & & & -1.95 & & & & & & & & \\
\hline Moraine & & & & & & & & & & & -0.0535 & & & & & \\
\hline DrainDens & & 0.655 & & & & & & 3.62 & -0.367 & 17 & & & & 44.4 & & \\
\hline Pmean & & & & & & & 0.272 & & $-1.36 \mathrm{E}-05$ & -0.00335 & 0.00127 & & & & & \\
\hline Tmean & & & & & & & & & & & & & & 2.93 & & -0.00475 \\
\hline PSI & & & & & & & & 26 & & & & & & & & \\
\hline Al & -17.3 & & & -48.2 & 0.0232 & & & & & & 1.18 & & & & & \\
\hline $\mathrm{Cz}$ & -0.425 & & 0.414 & -7.86 & & -0.185 & -5.91 & & 0.143 & & & & & & & \\
\hline CzMz & & 0.0241 & & & & & & 2.7 & & 0.432 & -0.449 & & & -6.69 & & \\
\hline $\mathrm{Mz}$ & & & & & & & & & & & & & & & & -0.0198 \\
\hline $\mathrm{pCm}$ & & & & & & & & & & & 0.649 & & & 13.3 & & \\
\hline $\mathrm{pCmi}$ & -3.51 & & & & & & & -1.25 & & & & & & & & \\
\hline Pz & & & & & & & & & & & & & & & & \\
\hline Pzi & & & & & & & & & & & & & & -9.61 & & \\
\hline Pzm & & & & 31.9 & & & & & & & 0.628 & & 0.132 & 24.9 & & -0.0133 \\
\hline PzpCmm & & & $2.67 \mid$ & & & & & & -0.0224 & & & & -0.0496 & & -0.0327 & \\
\hline
\end{tabular}




\begin{tabular}{|c|c|c|c|c|c|c|c|c|c|c|c|c|c|c|c|c|}
\hline & $\frac{0}{8}$ & ơ & Lㅇ & $\stackrel{\circ}{\circ}$ & 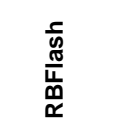 & 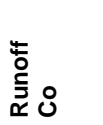 & 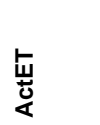 & $\begin{array}{l}\frac{3}{0} \\
\frac{0}{\omega}\end{array}$ & $\overline{\bar{u}}$ & 읖 & 离 & 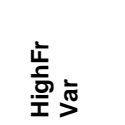 & 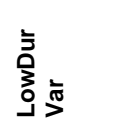 & 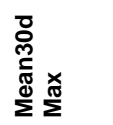 & $\begin{array}{l}\overline{\mathbf{m}} \\
\stackrel{0}{0}\end{array}$ & 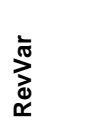 \\
\hline$\sqrt{\text { (Intercept) }}$ & -4.23 & 0.852 & 1.44 & -27 & 0.147 & 0.529 & 603 & 4.3 & 0.255 & 6.91 & \begin{tabular}{l|}
-0.255 \\
\end{tabular} & 0.379 & \begin{tabular}{ll|}
0.565 \\
\end{tabular} & 23.5 & 0.0651 & 0.0808 \\
\hline area & & $-1.53 \mathrm{E}-06$ & & & $-3.62 \mathrm{E}-07$ & & & & & & & 3.81E-07 & & & & \\
\hline \multicolumn{17}{|l|}{ meanElev } \\
\hline stdElev & & & & -0.0457 & & & & -0.000957 & & 0.000173 & & -0.000211 & & & & \\
\hline meanSlope & & & 0.232 & 0.76 & & 0.0141 & -1.48 & 0.0635 & & & & & & & & \\
\hline Water & 42.5 & & & 163 & & 1.93 & -2080 & & & & & & & & & \\
\hline \multirow{2}{*}{\multicolumn{17}{|c|}{$\begin{array}{l}\text { Glacier } \\
\text { Urban }\end{array}$}} \\
\hline & & & & & & & & & & & & & & & & \\
\hline \multicolumn{17}{|l|}{ Forest } \\
\hline Agriculture & & & & & & & & & & 3.48 & 0.0287 & & & & 0.0573 & \\
\hline \multicolumn{17}{|l|}{ Pasture } \\
\hline Wetland & & & 4.4 & & & & & -2.27 & & & & & & -22 & & \\
\hline OpwithVeg & & & & & 0.102 & & -585 & & & & & & & & & \\
\hline \multicolumn{17}{|l|}{ OpwithoutVeg } \\
\hline $\begin{array}{l}\text { Irrigated } \\
\end{array}$ & & & & & & & 379 & & & & 3.47 & & & & & \\
\hline \multirow{2}{*}{\multicolumn{17}{|c|}{$\begin{array}{l}\text { Coarse } \\
\text { Medium }\end{array}$}} \\
\hline & & & & & & & & & & & & & & & & \\
\hline \multirow{2}{*}{\multicolumn{17}{|c|}{ Fine }} \\
\hline \multirow{2}{*}{\multicolumn{17}{|c|}{$\begin{array}{l}\text { Organic } \\
\text { NoTexture }\end{array}$}} \\
\hline & & & & & & & & & & & & & & & & \\
\hline \multirow{2}{*}{\multicolumn{17}{|c|}{\begin{tabular}{|l} 
Shallow \\
Moraine
\end{tabular}}} \\
\hline & & & & & & & & & & & & & & & & \\
\hline \multicolumn{17}{|l|}{ DrainDens } \\
\hline Pmean & 0.0267 & -0.000209 & & 0.0684 & & & & -0.00247 & & -0.00386 & & & & -0.0149 & & \\
\hline Tmean & & & & & & & & & & & 0.0791 & & & & 0.000764 & \\
\hline PSI & & 2.71 & & & 0.131 & & & & & & & 0.163 & & & & \\
\hline $\mathrm{Al}$ & & & & & & & & & & & & & 0.0887 & & & 0.0522 \\
\hline $\mathrm{Cz}$ & & & & & & & & -0.57 & & -2.57 & & & & -4.91 & & \\
\hline \multicolumn{17}{|l|}{$\mathrm{CzMz}$} \\
\hline Mz & & & 0.0488 & & & & & & & & & & & & & \\
\hline $\mathrm{pCm}$ & & & -0.112 & & & & & & -0.0689 & & & & 0.0909 & & & \\
\hline \multicolumn{17}{|l|}{ pCmi } \\
\hline $\mathrm{Pz}$ & & & & & & \\
\hline Pzi & & & & & & & & & -0.188 & & & & 0.114 & & & \\
\hline Pzm & & & & & & & & & & & & & & & & \\
\hline PzpCmm & & & & & & & & & & & & & & & & \\
\hline
\end{tabular}




\begin{tabular}{|c|c|c|c|c|c|c|c|c|c|c|c|c|c|c|c|c|}
\hline & $\frac{0}{0}$ & गे & 40 & \% & 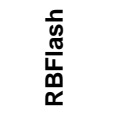 & 咅 & $\frac{\vec{w}}{\mathbb{4}}$ & $\begin{array}{l}\frac{3}{0} \\
\frac{\partial}{\omega}\end{array}$ & $\overline{\underline{\mathbf{m}}}$ & $\frac{a}{\underline{x}}$ & 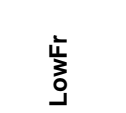 & $\begin{array}{l}\text { 壹 } \\
\text { 市 }\end{array}$ & 芌 & 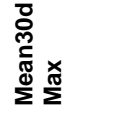 & $\begin{array}{l}\overline{0} \\
\overline{0} \\
\end{array}$ & 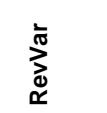 \\
\hline (Intercept) & -4.85 & 1.59 & 4.64 & 87.3 & 0.211 & 0.718 & 153 & 2.61 & 0.159 & 9.59 & 0.499 & 0.39 & $\begin{array}{ll}0.708 \\
\end{array}$ & -53.8 & $\begin{array}{l}0.0895 \\
\end{array}$ & 0.141 \\
\hline area & & & $3.21 \mathrm{E}-06$ & $-4.30 \mathrm{E}-05$ & $-3.47 \mathrm{E}-07$ & & & & & & $-1.31 \mathrm{E}-06$ & & & & & \\
\hline meanElev & & & & & & & & & & -0.00305 & & & & & & \\
\hline stdElev & & -0.000517 & & & & & & $-8.03 E-04$ & 0.00016 & & & & & 0.034 & & \\
\hline meanSlope & & & 0.165 & & & & & & & 0.227 & & & & & & \\
\hline Water & & -5.59 & & -102 & -1.85 & & & -13.4 & & -26.5 & & & & 19 & & \\
\hline \multicolumn{17}{|l|}{\begin{tabular}{|l} 
Glacier \\
Urban
\end{tabular}} \\
\hline Urban & & & & & & & & & & & -6.36 & & & & & \\
\hline \multicolumn{17}{|l|}{ Forest } \\
\hline Agriculture & -9.78 & & & & & & 309 & & & & & & & & & \\
\hline Pasture & & & & & & & & & & & & & -0.149 & & & \\
\hline Wetland & 24.7 & & & & & & -779 & & & & & & & & & \\
\hline \multirow{2}{*}{\multicolumn{17}{|c|}{$\begin{array}{l}\text { OpwithVeg } \\
\text { OpwithoutVeg }\end{array}$}} \\
\hline & & & & & & & & & & & & & & & & \\
\hline \multicolumn{17}{|l|}{ Irrigated } \\
\hline Coarse & & & & & & & & -1.34 & & & & & 0.0832 & & & -0.0183 \\
\hline Medium & -1.66 & & & & & & 52.5 & & & & & & & & & \\
\hline Fine & & & 0.164 & & -0.0121 & & & & & & 0.253 & & & & & \\
\hline Organic & & & & & & & & & & & & & -0.141 & & & \\
\hline \multicolumn{17}{|l|}{ NoTexture } \\
\hline Shallow & 7.93 & & & 47.6 & & 0.293 & -250 & & & & & & & & & \\
\hline \multicolumn{17}{|l|}{ Moraine } \\
\hline \multicolumn{17}{|l|}{ DrainDens } \\
\hline Pmean & 0.0224 & -0.000241 & & & & & 0.293 & & & -0.00496 & & & & & & \\
\hline Tmean & & & & & & & & & & & & & -0.00182 & 0.232 & & \\
\hline \multicolumn{17}{|l|}{$\begin{array}{l}\text { PSI } \\
\text { Al }\end{array}$} \\
\hline Al & & & -2.83 & -44.7 & & -0.284 & & & & & & & & 48.6 & & \\
\hline \multirow{2}{*}{\multicolumn{17}{|c|}{ CzMz }} \\
\hline & & & & & & & & & & & & & & & & \\
\hline Mz & & & & & & & & -0.565 & 0.0717 & & & -0.0174 & & & 0.00102 & \\
\hline \multicolumn{17}{|l|}{$\begin{array}{l}\mathrm{pCm} \\
\mathrm{pC} \mathrm{Cmi}\end{array}$} \\
\hline \multicolumn{17}{|l|}{$\mathbf{P z}$} \\
\hline \multicolumn{17}{|l|}{ Pzi } \\
\hline Pzm & & & & & & & & & & & & & & & & \\
\hline PzpCmm & & & & & & & & & & & & & & & & \\
\hline
\end{tabular}




\begin{tabular}{|c|c|c|c|c|c|c|c|c|c|c|c|c|c|c|c|c|}
\hline & $\frac{0}{8}$ & गे & 40 & 용 & 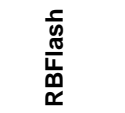 & 咅 & $\stackrel{5}{\underline{4}}$ & $\begin{array}{l}\frac{3}{0} \\
\frac{\partial}{\omega}\end{array}$ & 蒙 & 믇 & 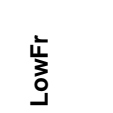 & 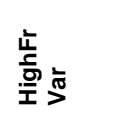 & 芌 & 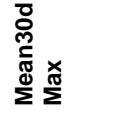 & $\begin{array}{l}\overline{0} \\
\overline{0} \\
\dot{0}\end{array}$ & 离 \\
\hline (Intercept) & -11.1 & -0.154 & 3.94 & \begin{tabular}{|c|}
-36.5 \\
\end{tabular} & 0.0154 & |0.777 & 350 & -1.38 & 0.328 & \begin{tabular}{l|}
-2.79 \\
\end{tabular} & 0.672 & 0.334 & $\begin{array}{ll}0.706 \\
\end{array}$ & \begin{tabular}{|l|}
-28.3 \\
\end{tabular} & 0.0819 & 0.151 \\
\hline area & & & $-2.45 E-06$ & & $-2.79 \mathrm{E}-07$ & & & & 3.06E-07 & & & & & & $-3.65 \mathrm{E}-08$ & \\
\hline \\
\hline stdElev & & -0.000685 & 0.00996 & & $-4.63 \mathrm{E}-05$ & & & 0.00144 & 0.000267 & 0.00143 & -0.00128 & & & & & \\
\hline meanSlope & & & & & & & & & & & & & & & & \\
\hline Water & & -5.25 & 10.6 & & -1.12 & & & & 0.771 & 22 & & 1.06 & & & & \\
\hline \multicolumn{17}{|l|}{ Glacier } \\
\hline Urban & & & & & & & & & & & & & -2.54 & & 0.167 & \\
\hline Forest & & & & & & -0.244 & & & & & & & & & & \\
\hline Agriculture & & & & & & -0.674 & & & & & & & & & & \\
\hline \multicolumn{17}{|l|}{ Pasture } \\
\hline Wetland & & & & 153 & & & & 4.23 & & 20.7 & & & & & & \\
\hline OpwithVeg & & & & 60.2 & & & & 6.29 & -0.209 & 12.9 & & & & & & \\
\hline OpwithoutVeg & 25.5 & & & & & & -804 & & & & & & & & & \\
\hline \multicolumn{17}{|l|}{ Irrigated } \\
\hline Coarse & & & & -10.1 & & 0.00195 & & & & & & & & & & \\
\hline Medium & -0.801 & & & -5.37 & & -0.0898 & 25.3 & & & & & & & & & \\
\hline Fine & & -0.0532 & -0.0762 & & & & & & 0.091 & & & & -0.0222 & & & \\
\hline Organic & & & & & 0.241 & & & & & & -0.705 & & & & & \\
\hline NoTexture & & & & & & & & 6.06 & & & & & & & & \\
\hline \multicolumn{17}{|l|}{ Shallow } \\
\hline Moraine & 9.27 & & & & & & -293 & & & & -0.429 & & & & & \\
\hline \multicolumn{17}{|l|}{ DrainDens } \\
\hline Pmean & 0.0254 & 0.000544 & & 0.0759 & 9.35E-05 & & 0.199 & -0.00269 & $-7.68 \mathrm{E}-05$ & -0.0046 & & -3.59E-05 & & & & \\
\hline Tmean & & -0.0281 & & & 0.0108 & & & 0.533 & -0.0054 & 1.03 & & -0.0113 & & & & \\
\hline PSI & & 2.26 & & & & & & & & & & & -0.0647 & 180 & & \\
\hline Al & & 0.668 & -2.25 & & & & & & & & & 0.161 & & & & \\
\hline Cz & & & & & -0.0385 & & & & & & & & & & 0.0357 & \\
\hline \multicolumn{17}{|l|}{$\begin{array}{l}\text { CzMz } \\
\text { Mz }\end{array}$} \\
\hline Mz & & & 0.469 & & & & & & & & & & & & & \\
\hline $\mathrm{pCm}$ & & 0.194 & & & & & & & -0.0889 & & & -0.0224 & & & & 0.0597 \\
\hline \multirow{2}{*}{\multicolumn{17}{|c|}{ pCmi }} \\
\hline \multicolumn{12}{|l|}{$\mathbf{P z}$} & & & & & \\
\hline Pzi & & & -2.04 & & & & & & & & 1.19 & & & & & -0.0134 \\
\hline Pzm & 4.06 & 0.407 & & & 0.0513 & 0.196 & -128 & 2.18 & & 3.85 & & & & -3.05 & & \\
\hline PzpCmm & & & & & & & & & & & & & & & & \\
\hline
\end{tabular}




\begin{tabular}{|c|c|c|c|c|c|c|c|c|c|c|c|c|c|c|c|c|}
\hline & $\frac{0}{8}$ & ơ & 용 & ஜூ & 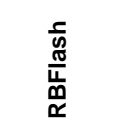 & 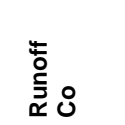 & 岀 & $\begin{array}{l}\frac{3}{0} \\
\frac{0}{\omega}\end{array}$ & $\underset{\boldsymbol{m}}{\overline{\boldsymbol{x}}}$ & $\frac{\underline{Q}}{\underline{x}}$ & 离 & 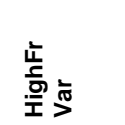 & 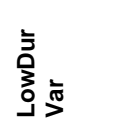 & 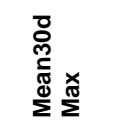 & $\begin{array}{l}\overline{\mathbf{m}} \\
\stackrel{0}{0}\end{array}$ & 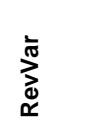 \\
\hline 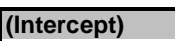 & -11.8 & \begin{tabular}{l|l|}
0.649 \\
\end{tabular} & -1.5 & 32.4 & 0.0971 & 0.345 & 349 & 3.54 & 0.318 & 5.19 & 0.591 & \begin{tabular}{l|l|}
0.582 \\
\end{tabular} & |0.693 & 11.4 & -0.0154 & 0.0841 \\
\hline area & $-2.41 \mathrm{E}-06$ & $-1.47 \mathrm{E}-06$ & & $-4.23 \mathrm{E}-05$ & $-3.35 \mathrm{E}-07$ & $-1.26 \mathrm{E}-07$ & 7.74E-05 & $-2.95 \mathrm{E}-06$ & 4.91E-07 & $-7.06 \mathrm{E}-06$ & & & & $-1.91 \mathrm{E}-05$ & & \\
\hline meanElev & & & & & & & & & & & & & & & & \\
\hline stdElev & & & & & & & & & & & & & & & 4.52E-05 & \\
\hline meanslope & & & & 1.51 & & & & & & & -0.0185 & & & & & \\
\hline Water & & & & & & & & & & & & & & & & \\
\hline Glacier & & & 2.96 & & & & & & & & & & & & 0.0411 & \\
\hline Urban & & 0.122 & 10.2 & & -0.25 & & & -10.6 & 1.42 & -6.89 & & & & 18.1 & & \\
\hline Forest & & -0.14 & & & -0.0562 & & & -2.32 & & & & & & & & \\
\hline Agriculture & & & & & & & & & & & & & & & & \\
\hline Pasture & & & & & & & & -3.42 & & -3.9 & & & & -18.7 & & \\
\hline Wetland & 42.9 & & & 186 & & 1.42 & -1240 & $\begin{array}{l}-1.13 \\
\end{array}$ & & 2.84 & -2.57 & & & 3.87 & & 0.286 \\
\hline OpwithVeg & & & & & & & & & & & 0.206 & & & & & \\
\hline OpwithoutVeg & & & 7.94 & & & & -557 & & & & & & -0.092 & & 0.0926 & \\
\hline Irrigated & & & & & & & & & & & & & & & -0.0743 & \\
\hline Coarse & & & & & & & & & & & & & & & & \\
\hline Medium & & & & -2.82 & & & & & & & & & & & & \\
\hline Fine & & 0.154 & & & -0.042 & & & & & & & & & & & \\
\hline Organic & & & & & & & & & & & & & & & & \\
\hline NoTexture & & -0.75 & & & 0.0507 & & & & -0.143 & & & & & & & \\
\hline Shallow & 11.9 & & & 36.7 & & 0.466 & -264 & & & & & & & & & \\
\hline Moraine & & & & & & & & -0.148 & & -1.44 & & & & -5.34 & & \\
\hline DrainDens & & & & & & & & & -0.156 & & & & & & & \\
\hline Pmean & 0.0245 & & 0.00453 & & & & 0.25 & & & & & $-1.97 \mathrm{E}-04$ & & & & \\
\hline Tmean & & & & & 0.0141 & & & & & & & & & & & \\
\hline PSI & & 2.93 & & & & & & & -0.269 & & & & & & & 0.249 \\
\hline Al & & & & & & & & & & & & & & & 0.0881 & \\
\hline $\mathrm{Cz}$ & & & & -21 & -0.0534 & & & & & & & & & & & \\
\hline CzMz & & 0.244 & 5.92 & & & & & & 0.113 & & & & & & & \\
\hline $\mathrm{Mz}$ & & & -0.0823 & & & & & & & & & -0.00485 & & & & \\
\hline $\mathrm{pCm}$ & 3.72 & & 0.388 & -6.91 & & 0.0998 & -116 & -0.306 & & -0.795 & & & & -3.08 & & \\
\hline pCmi & & & & -14.4 & & & & -0.444 & -0.0471 & -0.997 & 0.371 & & & -4.41 & & 0.0853 \\
\hline Pz & & & & & & & & & & & 0.123 & -0.0364 & -0.0785 & & & -0.0248 \\
\hline Pzi & 0.952 & & -2.32 & & & 0.121 & -30 & & -0.179 & & & & & & & \\
\hline Pzm & & & & & & & & & & & & & & & & \\
\hline PzpCmm & & 0.0729 & & & 0.064 & & & & & -0.703 & & & & & & \\
\hline
\end{tabular}




\section{E.2.2 Classification based on flow signatures}

Table M: Coefficients of the linear regression calibrated in group 1 of the FS classification. One model is calibrated for each flow signature and reads as a column in the table.

\begin{tabular}{|c|c|c|c|c|c|c|c|c|c|c|c|c|c|c|c|c|}
\hline & $\frac{0}{8}$ & Oج & $\stackrel{8}{0}$ & ஜூ & 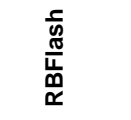 & 竞 & 惡 & $\begin{array}{l}\frac{3}{00} \\
\text { के }\end{array}$ & $\bar{\square}$ & 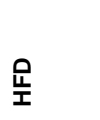 & 崩 & 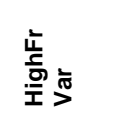 & 䓌 & 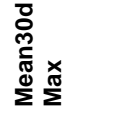 & $\begin{array}{l}\bar{\omega} \\
\overline{0} \\
\overline{0}\end{array}$ & 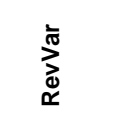 \\
\hline (Intercept) & -3.1 & 1.06 & 1.39 & $\begin{array}{l}5.78 \\
\end{array}$ & 0.195 & 0.672 & 279 & -3.13 & 0.194 & -2.81 & 0.523 & 0.38 & $\begin{array}{ll}0.709 \\
\end{array}$ & $\begin{array}{l}-27.6 \\
\end{array}$ & \begin{tabular}{|c|}
0.0907 \\
\end{tabular} & 0.147 \\
\hline area & & & & & $-3.71 \mathrm{E}-07$ & & & & & & & $2.16 \mathrm{E}-07$ & & & & $1.14 \mathrm{E}-07$ \\
\hline meanElev & & & $-4.02 \mathrm{E}-04$ & & & & & & 8.08E-05 & & & & & & & \\
\hline stdElev & & -0.00104 & 0.0111 & & & & & 0.000752 & & & & & & 0.00629 & & $-9.53 E-05$ \\
\hline meanSlope & & & & & & & & & & & & & -0.00324 & & & \\
\hline Water & & -3.6 & 12.7 & -30.5 & -1.77 & & & 6.77 & 0.879 & 28.6 & & & & 53.5 & & \\
\hline \multicolumn{17}{|l|}{ Glacier } \\
\hline Urban & & & & -162 & & & & & & & & & -2.45 & & & -1.09 \\
\hline Forest & -2.44 & & & & & & 87.4 & & & & & & & & & \\
\hline \multirow{2}{*}{\multicolumn{17}{|c|}{$\begin{array}{l}\text { Agriculture } \\
\text { Pasture }\end{array}$}} \\
\hline \multirow{2}{*}{\multicolumn{17}{|c|}{ Wetland }} \\
\hline & & & & & & & & & & & & & & & & \\
\hline \multirow{2}{*}{\multicolumn{17}{|c|}{ OpwithVeg }} \\
\hline OpwithoutVeg & & & 6.29 & & & & & & & & & & & & & \\
\hline Irrigated & & & -3.33 & & & & & & & & & 0.194 & & & & \\
\hline Coarse & & & & & & & & & & & -0.349 & & & & & \\
\hline \multirow{2}{*}{\multicolumn{17}{|c|}{\begin{tabular}{|l} 
Medium \\
Fine
\end{tabular}}} \\
\hline & & & & & & & & & & & & & & & & \\
\hline \multicolumn{17}{|l|}{ Organic } \\
\hline NoTexture & & & & & & & & & & & & & & & & 0.336 \\
\hline Shallow & 9.23 & 0.546 & & 28.7 & 0.0486 & 0.249 & -153 & & -0.144 & & & & & & & \\
\hline Moraine & & & & & & & & 4.32 & & & & & & 31.3 & & \\
\hline \multicolumn{17}{|l|}{ DrainDens } \\
\hline Pmean & 0.0248 & & & 0.06 & & & & & & & & & & & & \\
\hline Tmean & -0.845 & 0.0256 & & -1.76 & & & 30.7 & 0.525 & & 0.756 & & & & 3.6 & & \\
\hline PSI & 3.86 & & & & & 0.27 & -265 & & & & & & & & & \\
\hline Al & & & & -8.23 & & -0.282 & & & 0.0212 & & & & & & & \\
\hline $\mathrm{Cz}$ & -1.56 & & & & & -0.0509 & 27.2 & & & & & & & & & \\
\hline \multirow{2}{*}{\multicolumn{17}{|c|}{ Mz }} \\
\hline & & & & & & & & & & & & & & & & \\
\hline \multicolumn{17}{|l|}{$\mathrm{pCm}$} \\
\hline $\mathrm{pCmi}$ & & 0.0728 & & & & & & & & & & & 0.108 & & & \\
\hline $\mathrm{Pz}$ & 1.35 & & & & & & -32.2 & & & & 0.0531 & & & & & \\
\hline Pzi & & & & 9.54 & & & & & & & & & & & & \\
\hline Pzm & & & -2.38 & 27.2 & & 0.127 & & 2.51 & -0.136 & 5.18 & & & & & -0.0417 & \\
\hline PzpCmm & & & & 12.4 & & 0.0706 & & 0.172 & & 0.4 & & & & 1.94 & & \\
\hline
\end{tabular}




\begin{tabular}{|c|c|c|c|c|c|c|c|c|c|c|c|c|c|c|c|c|}
\hline & $\frac{0}{8}$ & ơ & $\stackrel{\text { 잉 }}{\circ}$ & ஜூ & 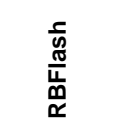 & $\begin{array}{l}\overline{\bar{\partial}} \\
\stackrel{\bar{z}}{\tilde{x}} 8\end{array}$ & 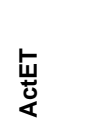 & $\begin{array}{l}\frac{3}{0} \\
\frac{d}{\omega}\end{array}$ & $\overline{\frac{1}{\omega}}$ & 잎 & 崩 & 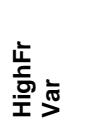 & 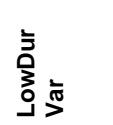 & 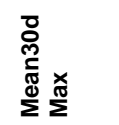 & $\begin{array}{l}\overline{\mathscr{\omega}} \\
\overline{0}\end{array}$ & 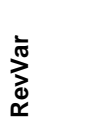 \\
\hline$\sqrt{\text { (Intercept) }}$ & 0.195 & $\mid 0.877$ & 4.63 & 7.12 & 0.186 & 0.701 & -6.15 & -2.19 & \begin{tabular}{l|l}
0.266 \\
\end{tabular} & 3.54 & -0.133 & 0.211 & $\begin{array}{ll}0.699 \\
\end{array}$ & -23.3 & \begin{tabular}{l|l}
0.0622 \\
\end{tabular} & 0.0859 \\
\hline area & & $-1.54 \mathrm{E}-06$ & & & $-3.81 \mathrm{E}-07$ & & & & & & & & & & & \\
\hline \multicolumn{17}{|l|}{ meanElev } \\
\hline stdElev & & & & & & & & -0.000649 & & & & & & -0.00451 & & \\
\hline meanSlope & & & 0.158 & & & & & & & 0.0115 & & & & & & \\
\hline Water & & & & & & & & & & & & 1.7 & & & -0.334 & \\
\hline \multicolumn{17}{|l|}{ Glacier } \\
\hline Urban & & & & & & & & & & & & & & & 0.541 & \\
\hline Forest & & & & & & & & -2.09 & & & & & & -13.5 & & \\
\hline Agriculture & -10.5 & & & -38.6 & & -0.251 & 330 & & & 1.69 & & & & & & \\
\hline Pasture & & & & & & & & & -0.113 & & & & & & -0.0718 & \\
\hline Wetland & & & & & -0.0329 & & & & & & & & & & & \\
\hline \multicolumn{17}{|l|}{ OpwithVeg } \\
\hline OpwithoutVeg & 21.6 & & 3.67 & & & & -680 & & & & & & & & & \\
\hline Irrigated & & & & & & & & & -0.0368 & 13.2 & 3.68 & & & & & \\
\hline Coarse & 0.534 & & & -3.53 & & 0.0473 & -16.9 & & & & & & & & -0.00964 & \\
\hline \multicolumn{17}{|l|}{ Medium } \\
\hline Fine & & & & & & & & & & & & & -0.0396 & & & \\
\hline \multicolumn{17}{|l|}{ Organic } \\
\hline NoTexture & & -1.01 & & & & & & & & & & 0.0681 & & & & \\
\hline Shallow & & -0.0505 & & & & & & & & & & & & & & \\
\hline \multicolumn{17}{|l|}{ Moraine } \\
\hline DrainDens & & & & & & & & & 0.01 & & & & & & -0.0342 & \\
\hline Pmean & 0.02 & $-2.04 \mathrm{E}-04$ & & 0.0592 & & & 0.368 & & & & & & & & & \\
\hline \multicolumn{17}{|l|}{ Tmean } \\
\hline PSI & & 2.77 & -2.76 & & & & & 25.3 & & & & & & 183 & 0.222 & 0.26 \\
\hline Al & -2.17 & & -1.97 & -6.87 & & -0.212 & 68.6 & & & & 0.527 & 0.141 & & & & \\
\hline $\mathrm{Cz}$ & -0.108 & & & -5.75 & & -0.00671 & 3.39 & & & & & & & & & \\
\hline \multirow{2}{*}{\multicolumn{17}{|c|}{ CzMz }} \\
\hline & & & & & & & & & & & & 0.00467 & & & & \\
\hline $\mathrm{pCm}$ & & & & & & & & & & & & & & & -0.011 & \\
\hline \multicolumn{17}{|l|}{$\mathrm{pCmi}$} \\
\hline $\mathrm{Pz}$ & & & & 1.51 & & & & & -0.0641 & 0.0796 & & & -0.079 & & & \\
\hline \multirow{2}{*}{\multicolumn{17}{|c|}{\begin{tabular}{|l} 
Pzi \\
Pzzm
\end{tabular}}} \\
\hline & & & & & & & & & & & & & & & & \\
\hline PzpCmm & & & & & & & & & & & & & & & & \\
\hline
\end{tabular}




\begin{tabular}{|c|c|c|c|c|c|c|c|c|c|c|c|c|c|c|c|c|}
\hline & $\frac{2}{8}$ & गे & 40 & \%ั & 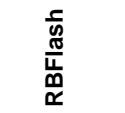 & 咅 & 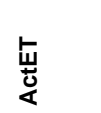 & $\begin{array}{l}\frac{3}{0} \\
\frac{0}{\omega}\end{array}$ & $\overline{\bar{n}}$ & 읃 & 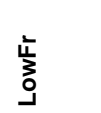 & 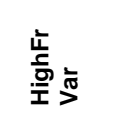 & 言 & 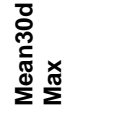 & $\begin{array}{l}\overline{0} \\
\overline{0} \\
0\end{array}$ & 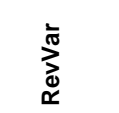 \\
\hline (Intercept) & -10.3 & 0.28 & -1.97 & -3.21 & 0.076 & 0.525 & 325 & \begin{tabular}{|l|}
-7.67 \\
\end{tabular} & \begin{tabular}{|l|}
0.177 \\
\end{tabular} & \begin{tabular}{|l|}
-13.6 \\
\end{tabular} & 0.329 & 0.575 & 0.774 & \begin{tabular}{l|l|}
-62.5 \\
\end{tabular} & $\mid-0.117$ & 0.224 \\
\hline area & & $-1.64 \mathrm{E}-06$ & & & $-3.85 \mathrm{E}-07$ & & & & & & & & & & & $1.28 \mathrm{E}-08$ \\
\hline meanElev & & & & & & & & & & & & & & & 7.44E-06 & \\
\hline stdElev & -0.000526 & & & & & & 0.0166 & 0.00288 & & & & & & 0.0217 & & \\
\hline meanSlope & 0.328 & -0.000377 & & & & & -10.4 & & & & & & & & & \\
\hline \multicolumn{17}{|l|}{ Water } \\
\hline \multirow{2}{*}{\multicolumn{17}{|c|}{\begin{tabular}{|l} 
Glacier \\
Urban
\end{tabular}}} \\
\hline & & & & & & & & & & & & & & & & \\
\hline Forest & 2.04 & & 0.197 & & & & -64.4 & & & & & & & & & \\
\hline Agriculture & & & & -48.5 & & -0.448 & & & & & & & & & & \\
\hline \multirow{2}{*}{\multicolumn{17}{|c|}{$\begin{array}{l}\text { Pasture } \\
\text { Wetland }\end{array}$}} \\
\hline & & & & & & & & & & & & & & & & \\
\hline \multirow{3}{*}{\multicolumn{17}{|c|}{$\begin{array}{l}\text { OpwithVeg } \\
\text { OppwithoutVeg } \\
\text { Irrigated }\end{array}$}} \\
\hline & & & & & & & & & & & & & & & & \\
\hline & & & & & & & & & -0.368 & -5.47 & & & & & & \\
\hline Coarse & & & & & & & & & 0.0582 & & & & & & & \\
\hline \multicolumn{17}{|l|}{ Medium } \\
\hline \multirow{2}{*}{\multicolumn{17}{|c|}{$\begin{array}{l}\text { Fine } \\
\text { Organic }\end{array}$}} \\
\hline \multirow{2}{*}{\multicolumn{17}{|c|}{$\begin{array}{l}\text { Organic } \\
\text { NoTexture }\end{array}$}} \\
\hline & & & & & & & & & & & & & & & & \\
\hline Shallow & & 0.116 & & & & 0.208 & & & & & & & & & & 0.0334 \\
\hline Moraine & & & & & & & & & & & & & & & -0.0296 & \\
\hline \multicolumn{17}{|l|}{ DrainDens } \\
\hline Pmean & 0.0228 & & 0.00556 & 0.064 & & & 0.281 & & & & & $-1.99 \mathrm{E}-04$ & & & 9.14E-05 & $-1.03 E-04$ \\
\hline Tmean & & 0.00388 & & & 0.0124 & & & & & & & & -0.00577 & & -0.0085 & \\
\hline PSI & & 2.28 & & & & & & 20.4 & & 34.7 & & & & 146 & 0.0855 & \\
\hline Al & & 0.383 & & & & & & 4.4 & & $9.77 \mid$ & & & & 32.3 & 0.152 & \\
\hline Cz & & & & & & & & & 0.174 & -4.76 & & & & & & \\
\hline CzMz & & & & & 0.272 & & & & 0.0601 & & & & & & & \\
\hline Mz & & & & & & & & 0.907 & 0.0758 & 0.788 & & & & 5.84 & 0.00687 & \\
\hline \multicolumn{17}{|l|}{$\begin{array}{l}\mathrm{pCm} \\
\mathrm{pCmi}\end{array}$} \\
\hline pCmi & & -0.18 & & & & & & & & & & & & & & \\
\hline \multicolumn{17}{|c|}{-0.0655} \\
\hline Pzi & -0.0354 & & & & & 0.0329 & 1.12 & & & & & & & & & \\
\hline Pzm & & & & & & & & 2.36 & & 1.96 & & & & 18.9 & & \\
\hline PzpCmm & & & & & & & & & & & & & & & & \\
\hline
\end{tabular}




\begin{tabular}{|c|c|c|c|c|c|c|c|c|c|c|c|c|c|c|c|c|}
\hline & $\frac{0}{8}$ & ơ & $\stackrel{\circ}{0}$ & ஜூ & 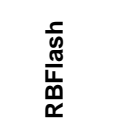 & 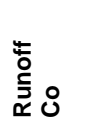 & 岀 & $\begin{array}{l}\frac{3}{0} \\
\frac{d}{\omega}\end{array}$ & $\frac{\bar{u}}{\infty}$ & 읖 & 訔 & 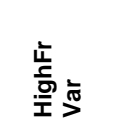 & 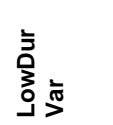 & 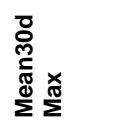 & $\begin{array}{l}\overline{\mathscr{\omega}} \\
\overline{0}\end{array}$ & 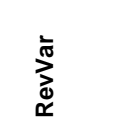 \\
\hline (Intercept) & -5.02 & 1.5 & 1.39 & -1.52 & 0.0951 & 0.699 & 130 & 3.9 & 0.256 & 7.5 & $\begin{array}{ll}0.468 \\
\end{array}$ & 0.41 & 0.832 & -2.13 & \begin{tabular}{l|}
0.0909 \\
\end{tabular} & 0.0954 \\
\hline area & & & & & $-3.51 \mathrm{E}-07$ & & & & & & & & & & & \\
\hline meanElev & & & & & $-6.75 \mathrm{E}-05$ & & -0.00103 & & & & & & & & & \\
\hline stdElev & & & & & & & & & & & & -0.000168 & & & & \\
\hline meanSlope & & & & & 0.00252 & & & & & & & & & & & \\
\hline Water & & & & & & & & -12 & & & & & & 104 & & \\
\hline Glacier & & & & & -0.939 & & & 10.5 & & & & & & & & \\
\hline Urban & & -3.89 & & & & & & & & & & & & -212 & & \\
\hline \multicolumn{17}{|l|}{ Forest } \\
\hline Agriculture & & & & -29.4 & & -0.233 & 172 & & & & & & & & & \\
\hline Pasture & & & & & & & -69.1 & & & & & & & & & \\
\hline \multicolumn{17}{|l|}{ Wetland } \\
\hline OpwithVeg & 17.9 & & & 49.3 & & 0.399 & -314 & & & & & & & & & \\
\hline \multirow{2}{*}{\multicolumn{17}{|c|}{$\begin{array}{l}\text { OpwithoutVeg } \\
\text { Irrigated }\end{array}$}} \\
\hline & & & & & & & & 6.85 & & & & & & & & \\
\hline Coarse & & & & -4.59 & & & & & & & & & & & & \\
\hline Medium & & & & & & & & & & & & & & & & -0.0197 \\
\hline Fine & & & -0.868 & & & & & & 0.0764 & & & & & & & \\
\hline \multicolumn{17}{|l|}{ Organic } \\
\hline NoTexture & & -0.8 & & & -0.205 & & & & & & & & & & -0.149 & \\
\hline Shallow & & & & & 0.0145 & & -203 & & & & & & & & & \\
\hline Moraine & 6.08 & & & & & 0.0507 & & & & & & & & & & \\
\hline \multicolumn{17}{|l|}{ DrainDens } \\
\hline Pmean & 0.0226 & -0.000279 & 0.00378 & 0.0596 & 0.000115 & & 0.261 & -0.00194 & $-3.22 \mathrm{E}-05$ & -0.00332 & & & -0.00017 & -0.0161 & $-4.28 \mathrm{E}-05$ & 3.52E-05 \\
\hline Tmean & & & & & & & 16.9 & & & & & & & 2.93 & & -0.0137 \\
\hline PSI & & & & & & & & & & & & & & & 0.223 & \\
\hline Al & -4.56 & & -1.55 & -8.26 & & -0.214 & & & & & & & & & & 0.126 \\
\hline $\mathrm{Cz}$ & & & & & & & & & & & -0.149 & & & & & \\
\hline \multirow{2}{*}{\multicolumn{17}{|c|}{$\begin{array}{l}\text { CzMz } \\
\text { Mz }\end{array}$}} \\
\hline & & & & & & & & & & & & & & & & \\
\hline \multicolumn{17}{|l|}{$\mathrm{pCm}$} \\
\hline \multirow{2}{*}{\multicolumn{17}{|c|}{$\begin{array}{l}\mathrm{pCmi} \\
\mathrm{Pz}\end{array}$}} \\
\hline & & & & & & & & & & & & & & & & \\
\hline \multicolumn{17}{|c|}{0.142} \\
\hline Pzm & & & & & & & & & & & & & & & & \\
\hline PzpCmm & & & & & & & & & & & -0.0237 & & & & & \\
\hline
\end{tabular}




\begin{tabular}{|c|c|c|c|c|c|c|c|c|c|c|c|c|c|c|c|c|}
\hline & $\frac{0}{8}$ & ơ & 용 & 용 & 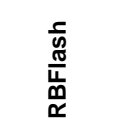 & 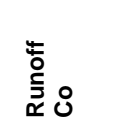 & 莸 & $\begin{array}{l}\frac{3}{0} \\
\frac{\mathbf{d}}{\omega}\end{array}$ & $\frac{\bar{u}}{\infty}$ & 잎 & 崩 & 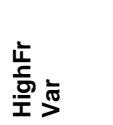 & 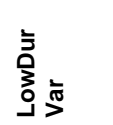 & 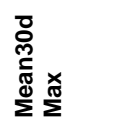 & $\begin{array}{l}\overline{\mathscr{\omega}} \\
\overline{0}\end{array}$ & 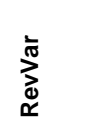 \\
\hline 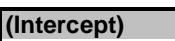 & -2.75 & $\mid 0.677$ & 4.35 & -43.4 & 0.21 & 0.413 & 86.7 & -0.201 & |0.297 & 1.41 & $-0.0811 \mid$ & \begin{tabular}{l|l}
0.399 \\
\end{tabular} & |0.667 & -44.2 & \begin{tabular}{l|l|}
0.0822 \\
\end{tabular} & 0.117 \\
\hline & & $-1.42 \mathrm{E}-06$ & & & $-3.46 \mathrm{E}-07$ & & & & & & & & & & & \\
\hline meanElev & & $-2.04 \mathrm{E}-04$ & -0.000632 & & & & & & & & & & & & & \\
\hline \multicolumn{17}{|l|}{ stdElev } \\
\hline meanSlope & & & 0.203 & & & 0.00766 & & & & & & & & & & \\
\hline Water & & & & & & & & & 0.511 & & & & & & & \\
\hline \multirow{2}{*}{\multicolumn{17}{|c|}{ Glacier }} \\
\hline & & & & & & & & & & & & & & & & \\
\hline Forest & & & & & -0.109 & & & 1.02 & & & & & & -4.43 & & 0.0536 \\
\hline Agriculture & -11.7 & & & & & -0.354 & 369 & 3.37 & & 4.81 & & & & & & \\
\hline Pasture & & 0.0976 & & & & & & & & & & -0.219 & & -16.1 & & \\
\hline Wetland & & & & 163 & & 1.05 & & & & & & & & & & \\
\hline OpwithVeg & & 0.356 & & 62 & 0.0772 & & & 6.56 & & 11.4 & & & & & & \\
\hline OpwithoutVeg & 22 & & & & & & -693 & & & & & & & & & \\
\hline Irrigated & & & & & & & & & & & & & & & & 0.123 \\
\hline Coarse & & & 1.93 & & & & & & & & & & & & & \\
\hline \multicolumn{17}{|l|}{ Medium } \\
\hline \multirow{2}{*}{\multicolumn{17}{|c|}{$\begin{array}{l}\text { Fine } \\
\text { Organic }\end{array}$}} \\
\hline & & & & & & & & & & & & & & & & \\
\hline NoTexture & & & & & & & & & & & & & -0.279 & & & \\
\hline \multirow{2}{*}{\multicolumn{17}{|c|}{\begin{tabular}{|l} 
Shallow \\
Moraine
\end{tabular}}} \\
\hline & & & & & & & & & & & & & & & & \\
\hline \multicolumn{17}{|l|}{ DrainDens } \\
\hline Pmean & 0.0219 & & & 0.0765 & & 0.000118 & 0.31 & & & & & & & & & \\
\hline Tmean & & & & & & & & & -0.00435 & & & & & 2.38 & & \\
\hline PSI & -1.8 & 2.78 & & 12.4 & & -0.135 & 57 & & -0.417 & & 2.51 & 0.0655 & & 174 & & \\
\hline AI & & & -2.37 & & & & & & 0.0546 & & & & & & & \\
\hline $\mathrm{Cz}$ & & & & & -0.025 & & & & & & & 0.0214 & 0.039 & & 0.0358 & \\
\hline CzMz & & & 6.81 & & & & & & & -5.16 & & & & 13.2 & & \\
\hline $\mathrm{Mz}$ & -1.46 & & & & 0.0226 & -0.0376 & 46.1 & & & & & & & & & \\
\hline $\mathrm{pCm}$ & & -0.0735 & & & & & & & & & & & & & 0.00267 & \\
\hline pCmi & & & & & & & & & & & 0.323 & & 0.19 & & & \\
\hline \multicolumn{17}{|l|}{$\mathrm{Pz}$} \\
\hline Pzi & & & & & & & & -0.0924 & & -0.255 & 1.05 & -0.0233 & & & & \\
\hline Pzm & & & & & & & & & & & & & 0.127 & & & \\
\hline PzpCmm & & & & & & & & & & & & & & & & \\
\hline
\end{tabular}




\begin{tabular}{|c|c|c|c|c|c|c|c|c|c|c|c|c|c|c|c|c|}
\hline & के & రి & \& & \% & 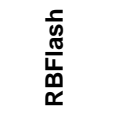 & 衣 & 离 & $\begin{array}{l}\frac{3}{0} \\
\frac{\grave{\nu}}{\omega}\end{array}$ & $\overline{\frac{\pi}{\infty}}$ & 品 & 崩 & 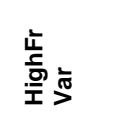 & 勇 & 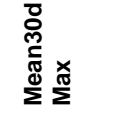 & $\begin{array}{l}\overline{0} \\
\overline{0} \\
\overline{0}\end{array}$ & 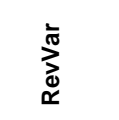 \\
\hline (Intercept) & -11.2 & 0.273 & 1.8 & -19.2 & 0.0397 & 0.439 & 354 & 2.12 & 0.361 & 4.42 & 0.561 & 0.427 & 0.691 & 7.68 & $\begin{array}{l}0.0468 \\
\end{array}$ & 0.0309 \\
\hline area & & $-1.61 \mathrm{E}-06$ & & $-2.37 \mathrm{E}-05$ & $-3.56 \mathrm{E}-07$ & & & & & & & & & & & \\
\hline meanElev & & & -0.000551 & & 3.55E-05 & & & & $-2.89 \mathrm{E}-05$ & & & $-5.07 \mathrm{E}-05$ & $-3.58 \mathrm{E}-05$ & & & \\
\hline stdElev & & & & & & & & & & & & $-9.77 E-05$ & & & & \\
\hline meanslope & & & 0.245 & & & & & & 0.00318 & & & & & & & \\
\hline Water & & & & & -1.08 & & & & & & & & & & & \\
\hline \multirow{2}{*}{\multicolumn{17}{|c|}{$\begin{array}{l}\text { Glacier } \\
\text { Urban }\end{array}$}} \\
\hline & & & & & & & & & & & & & & & & \\
\hline \multirow{2}{*}{\multicolumn{17}{|c|}{$\begin{array}{l}\text { Forest } \\
\text { Agriculture }\end{array}$}} \\
\hline & & & & & & & & & & & & & & & & \\
\hline Pasture & -5.51 & & & & & 0.0353 & 174 & & & & & & & & & \\
\hline Wetland & & 1.03 & & 180 & 0.442 & & & & & & & & & & & 0.315 \\
\hline \multicolumn{17}{|l|}{ OpwithVeg } \\
\hline OpwithoutVeg & & & & & & & & & & & & 0.1 & & & & \\
\hline \multicolumn{17}{|l|}{ Irrigated } \\
\hline \multirow{2}{*}{\multicolumn{17}{|c|}{\begin{tabular}{|l} 
Coarse \\
Medium
\end{tabular}}} \\
\hline & & & & & & & & & -0.0235 & & & & & & & \\
\hline \multicolumn{17}{|l|}{ Fine } \\
\hline Organic & & & & & 0.217 & & & & & & & -0.0906 & & & & \\
\hline NoTexture & & & & & & & & -2.46 & & -3.01 & & & & & -0.136 & \\
\hline Shallow & & & & & & & & & & & & & & & & 0.0443 \\
\hline Moraine & & & & & & & & & & & & & & & & 0.14 \\
\hline DrainDens & & & & $\begin{array}{l}-142 \\
-1471\end{array}$ & & & & & & & & & & & & \\
\hline Pmean & 0.0274 & & & 0.0774 & & & 0.135 & & $-8.56 \mathrm{E}-05$ & & & & & & & \\
\hline Tmean & & & & & 0.0141 & & & & & & & & & & & \\
\hline PSI & & 2.25 & & 30.3 & & & & & & & & & & & 0.236 & \\
\hline Al & & 0.414 & & & & & & & -0.0217 & & & & & & & 0.0781 \\
\hline \multirow{2}{*}{\multicolumn{17}{|c|}{$\begin{array}{l}\mathrm{Cz} \\
\mathrm{Cz} \mathrm{Mz}\end{array}$}} \\
\hline & & & & & & & & & & & & & & & & \\
\hline Mz & & & & & & & & & & & -0.226 & -0.0148 & & & & \\
\hline \multirow{2}{*}{\multicolumn{17}{|c|}{$\begin{array}{l}\mathrm{pCm} \\
\mathrm{pCCmi}\end{array}$}} \\
\hline & & & & & & & & & & & & & & & & \\
\hline $\begin{array}{l}\text { Pz } \\
\text { Pzi }\end{array}$ & & & -1.97 & & 0.018 & & & & $-0.0 / 56$ & & & & & & & \\
\hline Pzm & & & & 30.4 & & & & & & & & & 0.0967 & 7.45 & & 0.000657 \\
\hline PzpCmm & & & & & & & & & & & & & & & & \\
\hline
\end{tabular}




\begin{tabular}{|c|c|c|c|c|c|c|c|c|c|c|c|c|c|c|c|c|}
\hline & $\frac{0}{0}$ & गे & 40 & \% & 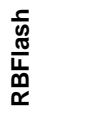 & 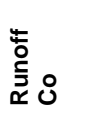 & $\stackrel{5}{\underline{4}}$ & $\begin{array}{l}\frac{3}{0} \\
\frac{\partial}{\omega}\end{array}$ & 离 & 믇 & 訔 & $\begin{array}{l}\text { 壹 } \\
\text { 市 }\end{array}$ & 芌 & 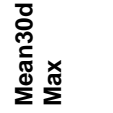 & $\begin{array}{l}\overline{0} \\
\overline{0} \\
\dot{0}\end{array}$ & 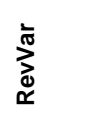 \\
\hline (Intercept) & -1.48 & 0.265 & 4.88 & -13.1 & 0.189 & 0.617 & 46.5 & -5.2 & 0.309 & 5.01 & \begin{tabular}{|c|}
-0.788 \\
\end{tabular} & \begin{tabular}{|l|}
0.377 \\
\end{tabular} & |0.687 & \begin{tabular}{l|l}
-60 \\
\end{tabular} & \begin{tabular}{|c|}
-0.00726 \\
\end{tabular} & 0.0546 \\
\hline area & & $-1.63 \mathrm{E}-06$ & & & & & & $-4.19 \mathrm{E}-06$ & & & & & & & $-4.56 \mathrm{E}-08$ & \\
\hline meanElev & & & 0.00268 & & & & & 0.0018 & & -0.00165 & & & & 0.00736 & & \\
\hline stdElev & & & & -0.0321 & & & & & & & & & & & & \\
\hline meanSlope & & 0.00326 & & & & & & & & & & & & & 0.0022 & \\
\hline Water & & & & & -1.71 & & & & & & & & & & & \\
\hline Glacier & & -1.39 & & & -0.399 & & & 16.7 & & & & & & 92 & & \\
\hline Urban & & & 25.2 & & 0.247 & & & -26.4 & 1.71 & -3.34 & & & -2.35 & 58.2 & & \\
\hline Forest & & & & & & & & & & & & & & & & 0.0602 \\
\hline Agriculture & -8.92 & & & -32.9 & & -0.555 & 282 & & 0.147 & & & & & & 0.00267 & 0.0307 \\
\hline \multicolumn{17}{|l|}{$\begin{array}{l}\text { Pasture } \\
\text { Wetland }\end{array}$} \\
\hline Wetland & & & & & & & & & -0.577 & & & & & & & \\
\hline OpwithVeg & & & & 43.8 & & & & & & & & & & & & \\
\hline OpwithoutVeg & & & & & & & & & & -1.72 & & & & & & \\
\hline \multicolumn{14}{|l|}{ |Irrigated } & & & \\
\hline Coarse & & & & -0.18 & & & & & & & & & & & & \\
\hline Medium & -5.93 & & & & & & 187 & & & & & & 0.0108 & & & \\
\hline Fine & -6.62 & & & & & & 209 & & & -1.25 & & & & & & \\
\hline Organic & & & 0.431 & & & 0.131 & & & & & & & & & & \\
\hline \multicolumn{17}{|l|}{ NoTexture } \\
\hline Shallow & & & & 23.3 & & & & & & 2.69 & & & & & & \\
\hline \multicolumn{17}{|l|}{ Moraine } \\
\hline \multicolumn{17}{|l|}{ DrainDens } \\
\hline Pmean & 0.0236 & & & 0.0664 & & & 0.256 & & & & & & & & & \\
\hline Tmean & & & & & & & & & -0.0124 & & 0.0837 & & & & -0.00349 & \\
\hline PSI & & 2.3 & -1.09 & & & & & & & & 2.51 & & & 140 & & 0.259 \\
\hline Al & & 0.421 & -2.61 & & & & & 6.08 & & & & & & 32.6 & 0.103 & \\
\hline Cz & -1.12 & & & -5.09 & & -0.0147 & 35.4 & & & & & & & & & \\
\hline CzMz & & & & & & & & & & & & & & 23.5 & & \\
\hline \multicolumn{17}{|l|}{ Mz } \\
\hline $\mathrm{pCm}$ & & & & & & & & & & & & 0.0356 & & & & \\
\hline pCmi & & & & & & & & & & & & 0.117 & & & & \\
\hline $\mathrm{Pz}$ & & & & & 0.034 & & & & & & & & & & & \\
\hline Pzi & & & -2.65 & & & & & & -0.138 & & 0.748 & & & & & \\
\hline Pzm & & & & & & & & & & & & & & & & \\
\hline PzpCmm & & & & & & & & & & & & & & & & \\
\hline
\end{tabular}




\begin{tabular}{|c|c|c|c|c|c|c|c|c|c|c|c|c|c|c|c|c|}
\hline & $\frac{0}{8}$ & ơ & 용 & ஜூ & $\begin{array}{l}\frac{5}{\mathscr{D}} \\
\frac{\pi}{\tilde{L}} \\
\frac{\underline{M}}{\tilde{x}}\end{array}$ & 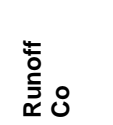 & 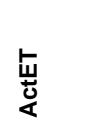 & $\begin{array}{l}\frac{3}{0} \\
\frac{0}{\omega}\end{array}$ & $\stackrel{\bar{u}}{\boldsymbol{~}}$ & 只 & 崩 & 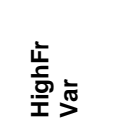 & 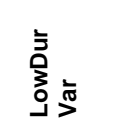 & 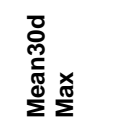 & $\begin{array}{l}\overline{\mathbf{m}} \\
\stackrel{0}{0}\end{array}$ & 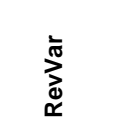 \\
\hline 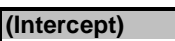 & \begin{tabular}{|c|}
-11.7 \\
\end{tabular} & 0.456 & 1.43 & \begin{tabular}{|l|}
-16.3 \\
\end{tabular} & 0.186 & 0.588 & 369 & -6.56 & 0.228 & -1.81 & -0.237 & 0.58 & \begin{tabular}{l|l}
0.703 \\
\end{tabular} & -49.7 & 0.104 & 0.163 \\
\hline area & & $-1.46 \mathrm{E}-06$ & $-1.70 \mathrm{E}-06$ & $-3.64 \mathrm{E}-05$ & & & & $-6.29 \mathrm{E}-09$ & $5.19 \mathrm{E}-07$ & & $-1.40 \mathrm{E}-06$ & & & & & $1.24 \mathrm{E}-08$ \\
\hline meanElev & -0.000872 & & & -0.00342 & & $1.65 \mathrm{E}-05$ & 0.0275 & & & & & & $-3.96 \mathrm{E}-05$ & & & \\
\hline stdElev & & & 0.00912 & & & & & & & -0.00109 & & & & -0.00179 & $-2.17 \mathrm{E}-05$ & \\
\hline meanSlope & 0.444 & & & 0.776 & 0.000288 & 0.00929 & -14 & 0.00607 & & & & & & & & \\
\hline Water & & -3.47 & & & $\begin{array}{r}-1.73 \\
\end{array}$ & & & 5.3 & & 28.5 & & & & 73.3 & & \\
\hline Glacier & & & & & & & & & & & & & & & & \\
\hline \multicolumn{17}{|l|}{ Urban } \\
\hline \multirow{2}{*}{\multicolumn{17}{|c|}{\begin{tabular}{|l} 
Forest \\
Agriculture
\end{tabular}}} \\
\hline & & & & & & & & & & & & & & & & \\
\hline Pasture & & & & & & & & & & & & & & & -0.0821 & -0.146 \\
\hline Wetland & & & & 160 & & 1.17 & & & & & & & & & & \\
\hline OpwithVeg & & & -0.0438 & & & & & & & & & -0.0447 & & & & \\
\hline OpwithoutVeg & & & & & & & & & & & & & 0.0158 & & & -0.0541 \\
\hline Irrigated & & & & & & & & & & & & & & & & \\
\hline Coarse & & & 1.88 & & & 0.0364 & & -0.398 & 0.137 & -4.28 & -0.651 & & & & & -0.0269 \\
\hline Medium & & 0.101 & & & & & & & & & & & & 1.03 & & \\
\hline Fine & & & & & & & & & & & & & -0.0412 & -1.36 & & \\
\hline \multicolumn{17}{|l|}{ Organic } \\
\hline NoTexture & 46 & & & & & & -1450 & & & & & & & & & \\
\hline Shallow & & & & & 0.0421 & & & & & & & & & & & \\
\hline Moraine & & & & & & & & 1.9 & & & & & & & & \\
\hline \multicolumn{17}{|l|}{ DrainDens } \\
\hline Pmean & 0.0226 & & 0.00201 & 0.0716 & & & 0.286 & & & & & $-1.97 \mathrm{E}-04$ & & & & \\
\hline Tmean & & 0.0189 & & -1.74 & & & & 0.413 & & 0.745 & & & & 2.45 & & \\
\hline PSI & & 2.79 & & & & & & 23.8 & & & & & -0.00399 & 176 & & \\
\hline AI & & & -1.57 & & & -0.223 & & & & & 0.684 & & & & & \\
\hline \multirow{2}{*}{\multicolumn{17}{|c|}{$\begin{array}{l}\mathrm{Cz} \\
\mathrm{CzMz}\end{array}$}} \\
\hline & & & & & & & & & & & & & & & & \\
\hline Mz & & & & & & & & & & & & & & & 0.00252 & \\
\hline $\mathrm{pCm}$ & 3.33 & & & & & 0.0928 & -105 & & & & & & & & & \\
\hline \multicolumn{17}{|l|}{$\mathrm{pCmi}$} \\
\hline Pz & & & & & & & & & & & & & & & & \\
\hline \multirow{2}{*}{\multicolumn{17}{|c|}{0.0626}} \\
\hline Pzm & & & & & & & & & & & & & & & & \\
\hline PzpCmm & & & & & & & & & & & & & & & & \\
\hline
\end{tabular}


Table U: Coefficients of the linear regression calibrated in group 10 of the FS classification. One model is calibrated for each flow signature and reads as a column in the table.

\begin{tabular}{|c|c|c|c|c|c|c|c|c|c|c|c|c|c|c|c|c|}
\hline & $\frac{0}{8}$ & ơ & 용 & ஜூ & 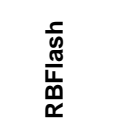 & 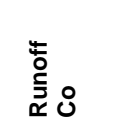 & 岀 & $\begin{array}{l}\frac{3}{0} \\
\frac{0}{\omega}\end{array}$ & $\stackrel{\bar{u}}{\boldsymbol{~}}$ & 只 & 崩 & 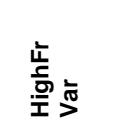 & 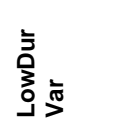 & 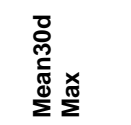 & $\begin{array}{l}\overline{\mathbf{n}} \\
\overline{0}\end{array}$ & 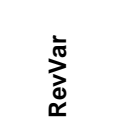 \\
\hline 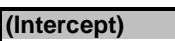 & -4.25 & 1.3 & -2.04 & -2.79 & 0.226 & 0.643 & 134 & 2.5 & 0.391 & 5.19 & 0.635 & \begin{tabular}{l|l|}
0.218 \\
\end{tabular} & |0.843 & 8.1 & 0.0431 & 0.163 \\
\hline area & $-8.01 \mathrm{E}-06$ & & & $-3.18 \mathrm{E}-05$ & $-3.13 \mathrm{E}-07$ & $-1.47 \mathrm{E}-07$ & 0.000253 & $-2.06 \mathrm{E}-06$ & $3.82 \mathrm{E}-07$ & & & & & $-1.28 \mathrm{E}-05$ & & \\
\hline meanElev & & & & & $-4.24 \mathrm{E}-05$ & 0.000218 & & & & & -0.000361 & $-1.21 \mathrm{E}-05$ & & & & \\
\hline stdElev & 0.00741 & -0.00105 & & & & & -0.234 & -0.00116 & & -0.00421 & & & & -0.00436 & & $-9.91 \mathrm{E}-05$ \\
\hline meanSlope & & & & & & & & & & & & & & 0.0386 & & \\
\hline Water & 26.1 & -5.74 & 22.3 & & -2.03 & & -823 & -10.5 & 0.844 & -21.9 & & 1.67 & & -43.8 & & 0.651 \\
\hline Glacier & & & 24.1 & 3.91 & & & & & 0.594 & & & & & & & \\
\hline Urban & & & & & & & & & & & & & -2.55 & & & \\
\hline \multicolumn{17}{|l|}{ Forest } \\
\hline Agriculture & -12.3 & & & -48.9 & & & 388 & & & & & & & & & \\
\hline Pasture & & & & & & & & -1.96 & & -3.17 & & & & & & -0.127 \\
\hline Wetland & & & & & & & & & -0.453 & & & & & & & \\
\hline OpwithVeg & & & & & & & & & & & & -0.0316 & & & & \\
\hline OpwithoutVeg & & -0.409 & & & & & & & & & & 0.13 & & & & \\
\hline Irrigated & & -0.400 & & 15.2 & & & & 6.51 & -0.162 & 12.8 & & & & 59.2 & & \\
\hline Coarse & & -0.475 & & & -0.0949 & & & & & & & & & & & -0.0216 \\
\hline \multirow{2}{*}{\multicolumn{17}{|c|}{\begin{tabular}{|l|} 
Medium \\
Fine
\end{tabular}}} \\
\hline & & & & & & & & & & & & & & & & \\
\hline \multicolumn{17}{|l|}{ Organic } \\
\hline NoTexture & & & & & & & & & & & & 0.0377 & & & & 0.129 \\
\hline Shallow & & 0.508 & & & & & & 0.524 & & 2.03 & & & & & & \\
\hline Moraine & & & & & & & & & & & & & 0.00128 & & & \\
\hline DrainDens & & 0.838 & & & -0.609 & -1.94 & & & & & & & & & & \\
\hline Pmean & 0.0221 & & 0.00505 & 0.0639 & 0.000111 & & 0.302 & & $-7.38 \mathrm{E}-05$ & & & & -0.00016 & & & \\
\hline \multirow{2}{*}{\multicolumn{17}{|c|}{$\begin{array}{l}\text { Tmean } \\
\text { PSI }\end{array}$}} \\
\hline & & & & & & -0.00601 & & & -0.302 & & & & & & 0.232 & \\
\hline Al & & & & & & & & & -0.051 & & & 0.143 & & & & \\
\hline $\mathrm{Cz}$ & & & & & & & & & 0.16 & & & & & & & \\
\hline CzMz & 0.863 & & & -19 & & 0.056 & -27.2 & & & & & & & & & \\
\hline $\mathrm{Mz}$ & & -0.172 & & & -0.0218 & & & & & & & & & & & \\
\hline \multicolumn{17}{|l|}{$\mathrm{pCm}$} \\
\hline pCmi & & & & & & & & -0.658 & & -0.842 & & & 0.0741 & -1.28 & & \\
\hline Pz & & & & & & & & -0.118 & & -0.0238 & & & & & & \\
\hline \multicolumn{17}{|l|}{ Pzi } \\
\hline Pzm & & 0.25 & & & -0.0151 & & & & & & & & & & & \\
\hline PzpCmm & & & & & & & & & & 0.132 & 0.385 & & & & & \\
\hline
\end{tabular}




\begin{tabular}{|c|c|c|c|c|c|c|c|c|c|c|c|c|c|c|c|c|}
\hline & $\frac{0}{8}$ & ơ & 용 & ஜூ & 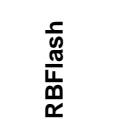 & $\begin{array}{l}\overline{\bar{\partial}} \\
\stackrel{\bar{z}}{\tilde{x}} 8\end{array}$ & 莸 & $\begin{array}{l}\frac{3}{0} \\
\frac{0}{\omega}\end{array}$ & $\stackrel{\bar{u}}{\boldsymbol{~}}$ & $\frac{\underline{Q}}{\underline{x}}$ & 崩 & 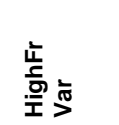 & 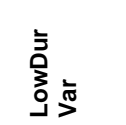 & 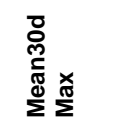 & $\begin{array}{l}\overline{\mathscr{\omega}} \\
\overline{0}\end{array}$ & 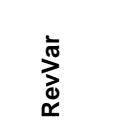 \\
\hline 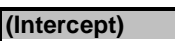 & 23.8 & 1.23 & 2.64 & -7.17 & 0.171 & 0.573 & \begin{tabular}{|l|}
39.7 \\
\end{tabular} & -3.85 & \begin{tabular}{l|l}
0.288 \\
\end{tabular} & -6.62 & \begin{tabular}{l|l}
0.0622 \\
\end{tabular} & 0.382 & $\mid 0.677$ & -49.1 & \begin{tabular}{|l|}
0.0894 \\
\end{tabular} & 0.166 \\
\hline area & & $-1.62 \mathrm{E}-06$ & & $-3.03 E-05$ & $-2.72 E-07$ & & & $-2.30 \mathrm{E}-06$ & & $-5.55 \mathrm{E}-06$ & & $2.19 \mathrm{E}-07$ & $-7.97 \mathrm{E}-09$ & $-1.61 \mathrm{E}-05$ & & \\
\hline meanElev & & & & & $-6.14 \mathrm{E}-05$ & & & & $2.28 \mathrm{E}-05$ & & -0.000533 & & & -0.00432 & & $-3.11 \mathrm{E}-05$ \\
\hline stdElev & & & & & -0.00013 & & & & & & & & & & $-1.05 \mathrm{E}-05$ & \\
\hline meanslope & & & & & 0.00703 & & & & 0.00173 & & & & & 1.21 & & \\
\hline Water & & & & & -1.28 & & & & & & & & & & & \\
\hline Glacier & 96.7 & & & & & & & & & & & & & & & \\
\hline Urban & & & & & 0.602 & & & & & & & & & & & -1.23 \\
\hline \multirow{2}{*}{\multicolumn{17}{|c|}{\begin{tabular}{|l} 
Forest \\
Agriculture
\end{tabular}}} \\
\hline & & & & & & & & & & & & & & & & \\
\hline Pasture & & & & & 0.156 & & & & & & & & & & & \\
\hline Wetland & & & -22.7 & & & & & & & & -1.95 & & & & & \\
\hline OpwithVeg & 14.6 & & & 51.6 & & 0.6 & -528 & & & & & & & & & \\
\hline OpwithoutVeg & & & & 47.5 & & & & & & & & & & & & \\
\hline Irrigated & & & & & & & & & 0.178 & & & & & & 0.161 & \\
\hline \multicolumn{17}{|l|}{ Coarse } \\
\hline Medium & & & -0.866 & & & & & & -0.0295 & & & & & & & \\
\hline Fine & & 0.0268 & & & & & & & & & & & & -20.9 & & \\
\hline \multicolumn{17}{|l|}{ Organic } \\
\hline NoTexture & & & 16.4 & & & & & & & & & & & & & \\
\hline \multicolumn{17}{|l|}{ Shallow } \\
\hline Moraine & & & & & & & & 1.05 & & 2.09 & -0.752 & & & & & \\
\hline DrainDens & & & & & & & & & & & & & & & & -0.0319 \\
\hline Pmean & & & 0.00302 & 0.0564 & & & 0.366 & & & & 0.000129 & & & & & \\
\hline Tmean & 0.841 & & & & & -0.0233 & & & -0.0065 & & & & & & & \\
\hline PSI & 18.5 & & & 24.4 & & & -270 & & -0.458 & & 3.05 & & & & & \\
\hline Al & -20.3 & & -2.41 & -16.8 & & & 214 & 5.89 & 0.0806 & 11 & & & & 53.8 & & \\
\hline $\mathrm{Cz}$ & & -0.166 & 1.65 & & & & & -2.85 & & -5.89 & -0.31 & & & -21.6 & & -0.00504 \\
\hline CzMz & & -0.0758 & 5.29 & & & 0.445 & & 8.31 & 0.127 & 14.4 & & & & 18.8 & & \\
\hline \multicolumn{17}{|l|}{$\mathrm{Mz}$} \\
\hline $\mathrm{pCm}$ & & & & 10.8 & & -0.00845 & & -0.83 & & -1.52 & 0.35 & & & & & \\
\hline \multicolumn{17}{|l|}{ pCmi } \\
\hline Pz & -1.61 & & & 7.4 & & & 5.53 & & & & & & & & & \\
\hline Pzi & & & & & & & & & & & 0.992 & & & & & \\
\hline \multicolumn{17}{|l|}{ Pzm } \\
\hline PzpCmm & & & & & & & & & & & & & & 6.35 & & \\
\hline
\end{tabular}




\section{E.2.3 Classification based on catchment descriptors}

Table W: Coefficients of the linear regression calibrated in group 1 of the CD classification. One model is calibrated for each flow signature and reads as a column in the table.

\begin{tabular}{|c|c|c|c|c|c|c|c|c|c|c|c|c|c|c|c|c|}
\hline & $\frac{0}{8}$ & ơ & \& & 8 & 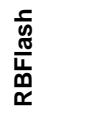 & 范 & 惡 & $\begin{array}{l}\frac{3}{0} \\
\text { के }\end{array}$ & $\bar{\square}$ & 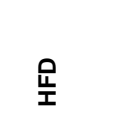 & 崩 & 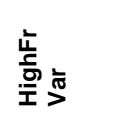 & 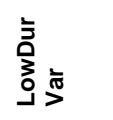 & 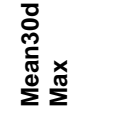 & $\begin{array}{l}\bar{\omega} \\
\overline{0} \\
\overline{0}\end{array}$ & 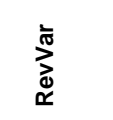 \\
\hline (Intercept) & -8.33 & 0.991 & 0.212 & $\begin{array}{ll}19.6 \\
\end{array}$ & 0.189 & 0.736 & 446 & -2.68 & |0.257 & \begin{tabular}{l|l|}
-4.36 \\
\end{tabular} & \begin{tabular}{|l|}
-0.043 \\
\end{tabular} & |0.357 & 0.67 & -49.9 & 0.0759 & 0.169 \\
\hline area & & & $4.22 \mathrm{E}-06$ & & & & & & 3.95E-07 & & & & & & & \\
\hline \multirow{2}{*}{\multicolumn{17}{|c|}{$\begin{array}{l}\text { meanElev } \\
\text { stdElev }\end{array}$}} \\
\hline & 0.0105 & & & & & 0.000231 & -0.123 & 4.96E-04 & & & & & & & & \\
\hline meanSlope & & & 0.15 & & & & & & & -0.00399 & & & & & 0.000738 & \\
\hline Water & & & 18.7 & 42.5 & -1.5 & & & & 0.928 & & & 1.39 & & 72.2 & & \\
\hline \multicolumn{17}{|l|}{ Glacier } \\
\hline Urban & -94.5 & 0.0108 & & & & & 2740 & & 1.35 & & & & & & 0.564 & \\
\hline \multirow{2}{*}{\multicolumn{17}{|c|}{$\begin{array}{l}\text { Forest } \\
\text { Agriculture }\end{array}$}} \\
\hline & & & & & & & & & & & & & & & & \\
\hline Pasture & -4.07 & & & -14.3 & & & 253 & & & & & & & & & \\
\hline Wetland & & 0.327 & & & & & & -2.86 & & 0.367 & & & & & & \\
\hline \multicolumn{17}{|l|}{ OpwithVeg } \\
\hline OpwithoutVeg & & & & & & & & & 0.183 & & & 0.00918 & & & & \\
\hline \multirow{2}{*}{\multicolumn{17}{|c|}{$\begin{array}{l}\text { Irrigated } \\
\text { Coarse }\end{array}$}} \\
\hline & & & & & & & & & & & & & & & & \\
\hline \multicolumn{17}{|l|}{ Medium } \\
\hline Fine & & & & & & & & & & & & & -0.0136 & & & \\
\hline Organic & & & & & & & & & & -3.11 & & & & & & \\
\hline NoTexture & & & & & & & & -2.97 & & $\begin{array}{l}-7.3 \\
\end{array}$ & & & & & & \\
\hline Shallow & & & & & 0.0383 & & & -2.26 & -0.0504 & -3.99 & & -0.132 & & & & \\
\hline Moraine & & -0.603 & & & & & & & & & & & 0.0606 & & & \\
\hline \multicolumn{17}{|l|}{ DrainDens } \\
\hline Pmean & 0.0242 & -0.000306 & 0.00353 & 0.0553 & & & & & & & & & & & $-5.19 E-05$ & \\
\hline Tmean & & & -0.122 & & 0.0107 & & & & & & & -0.00139 & & 2.45 & & -0.00392 \\
\hline PSI & & 2.94 & -3.42 & & 0.184 & & & 26.3 & -0.333 & 49.9 & 2.66 & 0.179 & & 177 & 0.245 & \\
\hline \multicolumn{17}{|c|}{-25.5} \\
\hline $\mathrm{Cz}$ & -4.56 & -0.342 & & & -0.0567 & & 119 & -0.928 & 0.15 & -2.26 & & 0.0425 & & & & \\
\hline \multicolumn{17}{|l|}{ CzMz } \\
\hline Mz & & & & & & & & & & & & & & & 0.0195 & \\
\hline $\mathrm{pCm}$ & & & & & & & & & & & & 0.00423 & & & & \\
\hline $\mathrm{pCmi}$ & & & & & & & & & & & & & 0.11 & & & 0.0917 \\
\hline \multicolumn{17}{|l|}{$\mathrm{Pz}$} \\
\hline Pzi & & & & & & 0.0542 & 50.1 & & & & & & & & & \\
\hline Pzm & & & & & 0.0144 & & & & & & & & & & & \\
\hline PzpCmm & & & & & & & & & & & & & & & & \\
\hline
\end{tabular}




\begin{tabular}{|c|c|c|c|c|c|c|c|c|c|c|c|c|c|c|c|c|}
\hline & $\frac{0}{8}$ & गे & 40 & \%ొ & 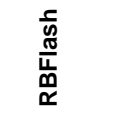 & 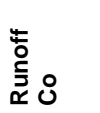 & $\stackrel{5}{\underline{4}}$ & $\begin{array}{l}\frac{3}{0} \\
\frac{2}{\omega}\end{array}$ & 蒿 & $\frac{\underline{u}}{\underline{x}}$ & $\sum_{0}^{\frac{1}{3}}$ & $\begin{array}{l}\text { 岕 } \\
\text { 히 }\end{array}$ & 芌 & 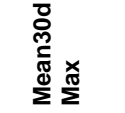 & $\begin{array}{l}\overline{0} \\
\overline{0}\end{array}$ & 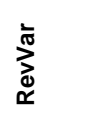 \\
\hline (Intercept) & -4.42 & 0.23 & 4.38 & 6.68 & $\overline{0.0497}$ & 0.694 & 107 & -6.8 & 0.331 & $\begin{array}{ll}-1.02 \\
\end{array}$ & \begin{tabular}{|c|}
-0.788 \\
\end{tabular} & 0.248 & |0.618 & -55.3 & $\mid-0.117$ & 0.0847 \\
\hline area & & $-9.30 \mathrm{E}-07$ & & & $-3.28 E-07$ & & & $-1.56 \mathrm{E}-06$ & 4.23E-07 & $-5.92 E-06$ & & & & $-8.97 \mathrm{E}-06$ & & \\
\hline \multicolumn{17}{|l|}{ meanElev } \\
\hline stdElev & & -0.00104 & 0.00864 & -0.0363 & & & & & & & & & & & & \\
\hline meanSlope & & 0.0207 & & 0.293 & 0.000916 & & & & & & & & & & & \\
\hline Water & & & & & -0.993 & & & & & & & 1.52 & & & & 1.04 \\
\hline Glacier & & & 13.3 & & & & & & & & & & & & & \\
\hline Urban & & & 12.7 & & & & 2170 & & 1.82 & -50.2 & & & & 23 & & -1.21 \\
\hline Forest & & & & -19.7 & & & 115 & & & & & & & & & \\
\hline Agriculture & -7.76 & & & -50.4 & & -0.235 & 398 & & & & & & & & & \\
\hline Pasture & & & -1.42 & & 0.13 & & & & -0.0754 & & & -0.118 & & & & -0.0773 \\
\hline Wetland & 18.2 & & & & & 0.33 & & & & & & & & & & \\
\hline OpwithVeg & 13 & & & & 0.237 & 0.385 & -366 & & & & & & -0.206 & & & \\
\hline \multirow{2}{*}{\multicolumn{17}{|c|}{$\begin{array}{l}\text { OpwithoutVeg } \\
\text { Irrigated }\end{array}$}} \\
\hline & & & & & & & & & & & & & & & & \\
\hline Coarse & & & & & & & & & & -4.06 & & & & & & \\
\hline \multicolumn{17}{|l|}{ Medium } \\
\hline Fine & & & & & & & & & & & & & & & -0.00972 & \\
\hline \multicolumn{17}{|l|}{ Organic } \\
\hline NoTexture & & 0.486 & & & & & & & & & & & & & & \\
\hline Shallow & & & & 30.6 & & & & & -0.086 & & & & & & 0.00266 & \\
\hline \multicolumn{17}{|l|}{ Moraine } \\
\hline \multicolumn{17}{|l|}{ DrainDens } \\
\hline Pmean & 0.0225 & & & 0.06 & & & 0.277 & & & & & & & & $1.10 \mathrm{E}-04$ & \\
\hline Tmean & & 0.0107 & 0.0608 & & 0.0109 & & & 0.0787 & -0.00922 & 0.705 & 0.0837 & & & 0.355 & -0.00872 & \\
\hline PSI & & 2.28 & & & & & & 20.3 & & & 2.51 & & & 147 & & \\
\hline Al & -2.12 & 0.374 & -2.79 & & & -0.21 & & 3.7 & & & & 0.13 & 0.0706 & 27.7 & 0.164 & 0.0573 \\
\hline Cz & & & & & -0.0298 & 0.0048 & & & 0.186 & & & -0.0135 & & & & \\
\hline CzMz & & & 3.23 & & & & & & & & & & & & & \\
\hline Mz & & & & & & & & & 0.101 & & & & & & & \\
\hline \multirow{2}{*}{\multicolumn{17}{|c|}{$\begin{array}{l}\mathrm{pCm} \\
\mathrm{pCmi}\end{array}$}} \\
\hline & & & & & & & & & & & & & & & & \\
\hline \multicolumn{17}{|l|}{$\mathrm{Pz}$} \\
\hline Pzi & & & & & & & & & & & 0.748 & & 0.126 & & & \\
\hline Pzm & & & & & & & & & & & & & & & -0.0177 & \\
\hline PzpCmm & & & & & & & & & & & & & & & & \\
\hline
\end{tabular}




\begin{tabular}{|c|c|c|c|c|c|c|c|c|c|c|c|c|c|c|c|c|}
\hline & $\frac{0}{8}$ & ơ & $\stackrel{20}{0}$ & 용 & 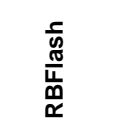 & 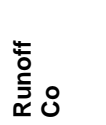 & 莸 & $\begin{array}{l}\frac{3}{0} \\
\frac{d}{\omega}\end{array}$ & $\frac{\bar{u}}{\infty}$ & 읖 & 崩 & 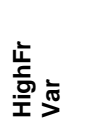 & 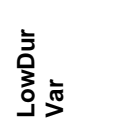 & 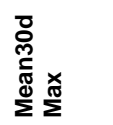 & $\begin{array}{l}\overline{\mathscr{\omega}} \\
\overline{0}\end{array}$ & 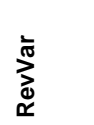 \\
\hline$\sqrt{\text { (Intercept) }}$ & -9.82 & 0.0596 & 2.53 & -29 & 0.162 & 0.718 & 310 & -12.8 & o.297 & -20.4 & -0.547 & 0.245 & 0.691 & -97.8 & -0.0527 & 0.0721 \\
\hline area & & $-1.82 \mathrm{E}-06$ & & & & & & & & & & & & & $-7.47 \mathrm{E}-08$ & \\
\hline meanElev & & & & & $-1.32 \mathrm{E}-05$ & & & & & & & & & & & \\
\hline stdElev & & & & & & & & & & & & & & & 6.17E-05 & \\
\hline meanSlope & & & & & & & & & & & & & & & & \\
\hline Water & & & & & & & & 24.7 & & & & & & 187 & & 1.15 \\
\hline Glacier & & & & & & & & & & & 2.36 & & & & & \\
\hline \multicolumn{17}{|l|}{ Urban } \\
\hline Forest & & & 1.24 & 8.18 & & & & & & & & & & & & \\
\hline \multirow{2}{*}{\multicolumn{17}{|c|}{$\begin{array}{l}\text { Agriculture } \\
\text { Pasture }\end{array}$}} \\
\hline Pasture & & & 0.371 & & & & & & & & & & & & & \\
\hline \multirow{2}{*}{\multicolumn{17}{|c|}{ Wetland }} \\
\hline & & & & & 0.125 & & & & & & & & & & & \\
\hline \multicolumn{17}{|l|}{ OpwithoutVeg } \\
\hline \multirow{2}{*}{\multicolumn{17}{|c|}{$\begin{array}{l}\text { Irrigated } \\
\text { Coarse }\end{array}$}} \\
\hline & & & & & & & & & & & & & & & & \\
\hline Medium & & & & & & & & 0.773 & -0.039 & 0.777 & 0.149 & & & 5.57 & 0.00676 & -0.0267 \\
\hline Fine & & -0.273 & & & & & & & & & & & & & & \\
\hline \multicolumn{17}{|l|}{ Organic } \\
\hline \multicolumn{17}{|l|}{ NoTexture } \\
\hline Shallow & 12.4 & & & 36.8 & 0.0289 & 0.293 & -390 & & & & & & -0.11 & & & \\
\hline Moraine & & & & & & & & & & & & & & & & \\
\hline \multicolumn{17}{|l|}{ DrainDens } \\
\hline Pmean & 0.0235 & 0.00038 & & 0.0669 & & & 0.259 & 0.00573 & & 0.00944 & & & & 0.0402 & 2.71E-05 & \\
\hline \multirow{2}{*}{\multicolumn{17}{|c|}{$\begin{array}{l}\text { Tmean } \\
\text { PSI }\end{array}$}} \\
\hline & & & & & & & & & -0.363 & & 2.02 & & & & & \\
\hline Al & & 0.765 & & & 0.0057 & -0.284 & & 7.99 & & 13.9 & 0.513 & 0.127 & & 57.4 & 0.0968 & 0.0615 \\
\hline $\mathrm{Cz}$ & & & & & & & & & 0.156 & & & & & & & \\
\hline \multirow{2}{*}{\multicolumn{16}{|c|}{$\mathrm{Mz}$}} & \\
\hline & & & & & & & & & & & & & & & & \\
\hline \multicolumn{17}{|l|}{$\mathrm{pCm}$} \\
\hline \multirow{2}{*}{\multicolumn{17}{|c|}{$\begin{array}{l}\mathrm{pCmi} \\
\mathrm{Pz}\end{array}$}} \\
\hline & & & & & & & & & & & & & & & & \\
\hline \multicolumn{17}{|l|}{$\begin{array}{l}\text { Pzi } \\
\text { Pzm }\end{array}$} \\
\hline Pzm & & & & & & & & & & & & & & & & \\
\hline PzpCmm & & & & & & & & & & & & & & & & \\
\hline
\end{tabular}




\begin{tabular}{|c|c|c|c|c|c|c|c|c|c|c|c|c|c|c|c|c|}
\hline & $\frac{0}{8}$ & రి & $\mathscr{0}$ & \% & 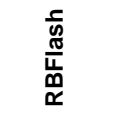 & 衣 & 离 & $\begin{array}{l}\frac{3}{0} \\
\frac{\vec{d}}{\omega}\end{array}$ & $\overline{\frac{\pi}{\infty}}$ & $\frac{\underline{u}}{\underline{x}}$ & 茨 & 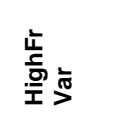 & 竎 & 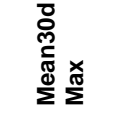 & $\begin{array}{l}\bar{\omega} \\
\overline{0} \\
\bar{\delta}\end{array}$ & 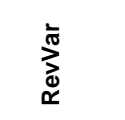 \\
\hline (Intercept) & 3.76 & 0.896 & 2.04 & -16.7 & 0.083 & 0.719 & 308 & -1.26 & 0.147 & -3.73 & \begin{tabular}{|c|}
-1.7 \\
\end{tabular} & 0.41 & \begin{tabular}{l|l}
0.673 \\
\end{tabular} & -17.2 & $\begin{array}{l}0.0915 \\
\end{array}$ & 0.188 \\
\hline area & & $-1.73 \mathrm{E}-06$ & & $-2.41 \mathrm{E}-05$ & & & & $-2.60 \mathrm{E}-06$ & 4.17E-07 & $-5.81 \mathrm{E}-06$ & & 2.19E-07 & & $-1.67 \mathrm{E}-05$ & & \\
\hline \multirow{2}{*}{\multicolumn{17}{|c|}{$\begin{array}{l}\text { meanElev } \\
\text { stdElev }\end{array}$}} \\
\hline & 0.00898 & & 0.0117 & & -0.000128 & & & & & & & & & & & $-8.93 E-05$ \\
\hline meanSlope & & & & & & & & & 0.0017 & & & & & & & \\
\hline Water & & & 17.5 & & -1.62 & & & & 1.38 & & & & & & & \\
\hline \multirow{2}{*}{\multicolumn{17}{|c|}{$\begin{array}{l}\text { Glacier } \\
\text { Urban }\end{array}$}} \\
\hline & & & & & & -1.61 & & & 1.57 & & & & & & & \\
\hline Forest & & & & & & & & & & & -0.691 & & & & -0.0199 & \\
\hline \multicolumn{17}{|l|}{ Agriculture } \\
\hline Pasture & & -0.178 & & & & 0.319 & & & & & & & & & & \\
\hline Wetland & & & & & 0.177 & & & & & 27.5 & & & & & & \\
\hline \multirow{2}{*}{\multicolumn{17}{|c|}{$\mid$\begin{tabular}{|l} 
OpwithVeg \\
OpwithoutVeg
\end{tabular}}} \\
\hline & & & & & & & & & & & & & & & & \\
\hline \multicolumn{17}{|c|}{ Irrigated } \\
\hline Coarse & -0.557 & & & & & -0.348 & -0.109 & & & & & & & & & \\
\hline Medium & & & & -14.2 & & -0.408 & & & -0.0179 & & & & & & & \\
\hline Fine & & & & & & -0.506 & & & & & -0.252 & & & & & \\
\hline \multirow{2}{*}{\multicolumn{17}{|c|}{$\begin{array}{l}\text { Organic } \\
\text { NoTexture }\end{array}$}} \\
\hline & & & & & & & -631 & & & & & & & & & \\
\hline \multicolumn{17}{|l|}{$\begin{array}{l}\text { Shallow } \\
\text { Moraine }\end{array}$} \\
\hline Moraine & & & & & & & & & & & 0.441 & & & & & \\
\hline DrainDens & & 2.43 & & & & & & 2.24 & & 4.3 & 0.613 & & & 32.9 & & \\
\hline Pmean & 0.0181 & & & 0.0731 & 0.000135 & & & & & & 0.00108 & & & & & \\
\hline Tmean & & & & & & & 24.8 & 0.339 & & 0.795 & & -0.00314 & & 2.3 & & -0.00466 \\
\hline PSI & & & -4.58 & & & & & & & & & & & & & \\
\hline \multicolumn{17}{|c|}{-7.33} \\
\hline $\mathrm{Cz}$ & & -0.205 & 0.15 & -20.3 & & & & & 0.173 & & -0.675 & & & & 0.0315 & \\
\hline CzMz & -2.85 & & & & & & 370 & & & & & & & & & \\
\hline Mz & & & & & & & & & 0.0868 & & & & & & & \\
\hline $\mathrm{pCm}$ & & & & & & & & & 0.00584 & & & & & & & 0.0451 \\
\hline \multirow{2}{*}{\multicolumn{17}{|c|}{$\begin{array}{l}\mathrm{pCmi} \\
\mathrm{Pz}\end{array}$}} \\
\hline \multirow{2}{*}{\multicolumn{17}{|c|}{\begin{tabular}{|l}
$\mathrm{Pz}$ \\
$\mathrm{Pzi}$
\end{tabular}}} \\
\hline & & & & & & & & & & & & & & & & \\
\hline Pzm & & & & & & & & & -0.0453 & & 0.249 & & 0.0786 & & & \\
\hline PzpCmm & & & & & & & & & & & & & & & -0.0195 & \\
\hline
\end{tabular}




\begin{tabular}{|c|c|c|c|c|c|c|c|c|c|c|c|c|c|c|c|c|}
\hline & $\frac{0}{8}$ & ग̊ & 용 & 용 & 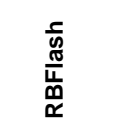 & 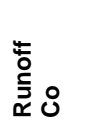 & 莸 & $\begin{array}{l}\frac{3}{0} \\
\frac{0}{\omega}\end{array}$ & $\stackrel{\bar{u}}{\boldsymbol{~}}$ & 읖 & 崩 & 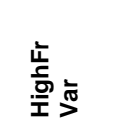 & 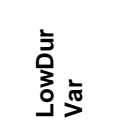 & 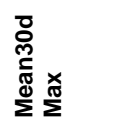 & $\begin{array}{l}\overline{\mathscr{\omega}} \\
\overline{0}\end{array}$ & 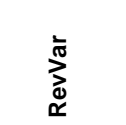 \\
\hline 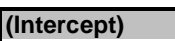 & -10.8 & 0.577 & 4.12 & \begin{tabular}{l|}
-3.42 \\
\end{tabular} & 0.0644 & 0.316 & 341 & -8.24 & 0.193 & -13.5 & 0.691 & 0.565 & 0.705 & -67.9 & \begin{tabular}{l|l|}
0.0375 \\
\end{tabular} & 0.11 \\
\hline area & & $-1.15 \mathrm{E}-06$ & & & $-2.76 \mathrm{E}-07$ & & & & $3.72 \mathrm{E}-07$ & & & & & & $-1.17 \mathrm{E}-08$ & \\
\hline meanElev & & & -0.000583 & & & & & & & 0.00106 & & & $-4.00 \mathrm{E}-05$ & 0.0057 & & \\
\hline stdElev & & -0.000507 & $\begin{array}{l}0.0102 \\
\end{array}$ & & $-6.35 \mathrm{E}-05$ & & & 0.00412 & $1.98 \mathrm{E}-04$ & 0.00222 & -0.00109 & & & 0.0211 & & $-7.10 \mathrm{E}-05$ \\
\hline meanSlope & 0.228 & & & & & 0.0129 & -7.18 & & & & & & & & & \\
\hline Water & & & 15.4 & -20.3 & -1.03 & & & & 0.499 & & & 1.1 & & & & \\
\hline \multicolumn{17}{|l|}{ Glacier } \\
\hline Urban & & & 10.9 & & & & & & & & -6.18 & & & & & \\
\hline Forest & & & & & -0.0481 & & & & & & & & & & & 0.0355 \\
\hline Agriculture & & & & -46.4 & & & & & & & & & & & & \\
\hline Pasture & & -0.0768 & & & & & & 3.98 & & & & & -0.197 & 30.4 & & -0.105 \\
\hline Wetland & & & & & & 1.78 & & & & & & & & & & \\
\hline OpwithVeg & & & & & & & & & & & & -0.0776 & & & & \\
\hline OpwithoutVeg & 18.9 & & & & & 0.438 & -596 & & & & & 0.15 & & & -0.0343 & \\
\hline Irrigated & & & & & & & & & 0.11 & & & & & & & \\
\hline \multirow{2}{*}{\multicolumn{16}{|c|}{\begin{tabular}{|l} 
Coarse \\
Medium
\end{tabular}}} & \\
\hline & & & & & & & & & & & & & 0.0405 & & & \\
\hline \multirow{2}{*}{\multicolumn{17}{|c|}{$\begin{array}{l}\text { Fine } \\
\text { Organic }\end{array}$}} \\
\hline & & & & & & & & & & & & & & & & \\
\hline \multicolumn{17}{|l|}{$\begin{array}{l}\text { Organic } \\
\text { NoTexture }\end{array}$} \\
\hline Shallow & & & 0.394 & & & & & & & & & -0.0217 & & & & \\
\hline Moraine & 10.4 & & -0.911 & & & & -327 & & & & & & & & & \\
\hline \multicolumn{17}{|l|}{ DrainDens } \\
\hline Pmean & 0.0232 & -0.000135 & & 0.0623 & $9.68 \mathrm{E}-05$ & & 0.267 & & & & & $-1.93 \mathrm{E}-04$ & & & & \\
\hline Tmean & & 0.0314 & & & 0.00807 & & & -0.0541 & -0.00224 & 0.125 & & & & -0.629 & & \\
\hline PSI & & 2.68 & & & & & & 20.1 & & 37.8 & & & & 142 & 0.232 & \\
\hline Al & & & -2.45 & & & & & 5.08 & 0.0353 & 7.54 & & & & 39.3 & & 0.0374 \\
\hline $\mathrm{Cz}$ & & & & & -0.0423 & & & & & & & 0.0185 & & & 0.0328 & \\
\hline \multicolumn{17}{|l|}{ CzMz } \\
\hline $\mathrm{Mz}$ & & & 0.581 & & & & & & & & & & & & & \\
\hline \multirow{2}{*}{\multicolumn{17}{|c|}{$\begin{array}{l}\mathrm{pCm} \\
\mathrm{pCmi}\end{array}$}} \\
\hline & & & & & & & & & & & & & & & & \\
\hline \multicolumn{17}{|l|}{$\mathrm{Pz}$} \\
\hline Pzi & & 0.201 & & & & & & -0.91 & -0.18 & & & & & -7.14 & & \\
\hline Pzm & 3.9 & 0.296 & & 36 & 0.025 & 0.108 & -123 & & -0.135 & & & & & & & \\
\hline PzpCmm & 1.12 & & & 12.4 & & & -35.3 & & & & & & & & & \\
\hline
\end{tabular}




\begin{tabular}{|c|c|c|c|c|c|c|c|c|c|c|c|c|c|c|c|c|}
\hline & $\frac{2}{8}$ & गे & 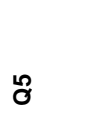 & \% & 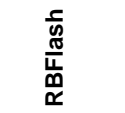 & 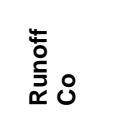 & $\frac{\bar{y}}{0}$ & $\begin{array}{l}\frac{3}{0} \\
\frac{0}{\omega}\end{array}$ & $\overline{\underline{\mathbf{m}}}$ & $\frac{a}{\underline{x}}$ & 崩 & $\begin{array}{l}\text { 岕 } \\
\text { 히 }\end{array}$ & 芌 & 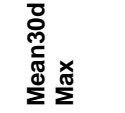 & $\begin{array}{l}\overline{0} \\
\overline{0} \\
0\end{array}$ & 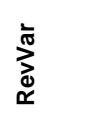 \\
\hline (Intercept) & -4.39 & 0.282 & \begin{tabular}{|c|}
7.68 \\
\end{tabular} & -32.6 & 0.0952 & 0.844 & 385 & -5.64 & 0.259 & -8.61 & 0.0623 & 0.228 & (0.832 & -25.2 & 0.042 & 0.057 \\
\hline area & & $-1.38 \mathrm{E}-06$ & & & $-3.65 \mathrm{E}-07$ & & & $9.75 \mathrm{E}-07$ & 4.46E-07 & $1.03 \mathrm{E}-06$ & & & & $6.75 \mathrm{E}-06$ & & \\
\hline meanElev & -0.00198 & & & 0.00998 & & 8.14E-06 & 0.0333 & -0.000419 & $1.72 \mathrm{E}-05$ & -0.00105 & -0.000391 & & & -0.00562 & $-5.22 \mathrm{E}-06$ & \\
\hline stdElev & -0.00021 & & & -0.0392 & & & 0.00989 & & & & & & & & $1.14 \mathrm{E}-05$ & \\
\hline meanSlope & 0.346 & 0.00299 & & 0.726 & 0.00216 & 0.00722 & -8.65 & & -0.000326 & & $9.02 \mathrm{E}-04$ & & & & & \\
\hline Water & & & & & -1.03 & & & & & & & 1.77 & & & & \\
\hline \multirow{2}{*}{\multicolumn{17}{|c|}{$\begin{array}{l}\text { Glacier } \\
\text { Urban }\end{array}$}} \\
\hline & & & & & & & & & & & & & & & & \\
\hline Forest & & -0.102 & & 8.35 & -0.0547 & -0.028 & & & 0.0313 & -1.79 & & & & & & \\
\hline \multicolumn{17}{|l|}{ Agriculture } \\
\hline \multirow{2}{*}{\multicolumn{17}{|c|}{$\begin{array}{l}\text { Pasture } \\
\text { Wetland }\end{array}$}} \\
\hline & & & & & & & & & & & & & & & -0.166 & \\
\hline \multirow{2}{*}{\multicolumn{17}{|c|}{$\begin{array}{l}\text { OpwithVeg } \\
\text { OpwithoutVeg }\end{array}$}} \\
\hline & & & & & & & & & & & & & & & & \\
\hline \multicolumn{17}{|l|}{ Irrigated } \\
\hline Coarse & 1.04 & & 0.607 & & & -0.0134 & -28 & & & & & & & & & -0.0211 \\
\hline \multicolumn{17}{|l|}{ Medium } \\
\hline Fine & & & & & & -0.142 & & & & & & & & & & \\
\hline \multirow{2}{*}{\multicolumn{17}{|c|}{$\begin{array}{l}\text { Organic } \\
\text { NoTexture }\end{array}$}} \\
\hline & & & & & & & & & & & & & & & & \\
\hline \multirow{2}{*}{\multicolumn{17}{|c|}{\begin{tabular}{|l|} 
Shallow \\
Moraine
\end{tabular}}} \\
\hline Moraine & & & & & & & & & & & & & & & & \\
\hline DrainDens & & & -4.51 & & & & & & -0.33 & & 0.24 & & & & 0.0273 & \\
\hline Pmean & 0.0245 & & & 0.0695 & & $-9.13 E-05$ & & & & & & & -0.00017 & & & \\
\hline Tmean & -0.882 & 0.00196 & & & 0.00871 & & 44.8 & 0.354 & & 0.681 & & & & & & \\
\hline PSI & 7.55 & 2.26 & & & 0.155 & & & 24.9 & & 50 & 2.8 & & & 184 & 0.256 & 0.212 \\
\hline Al & & 0.504 & -3.97 & & & -0.296 & -186 & & -0.021 & & & 0.133 & & & & 0.0404 \\
\hline \multirow{2}{*}{\multicolumn{17}{|c|}{ CzMz }} \\
\hline & & & & & & & & & & & & & & & & \\
\hline Mz & & & & & -0.0109 & & & & & & & & & & & \\
\hline \multicolumn{17}{|l|}{$\mathrm{pCm}$} \\
\hline pCmi & & -0.26 & -1.22 & & & & & & -0.0254 & & & & & & & \\
\hline $\begin{array}{l}\mathrm{Pz} \\
\mathrm{Pzi}\end{array}$ & & & & 11.8 & & & & & & -3.57 & & & & & & -0.0189 \\
\hline Pzi & & & & 6.9 & & & & & & -6.5 & & & 0.142 & -7.4 & & \\
\hline Pzm & & 0.213 & & & 0.0241 & & & 1.02 & & 1.05 & & & & -1.22 & & \\
\hline PzpCmm & & & & & & & & & & & & & & & & \\
\hline
\end{tabular}




\begin{tabular}{|c|c|c|c|c|c|c|c|c|c|c|c|c|c|c|c|c|}
\hline & $\frac{2}{8}$ & गे & 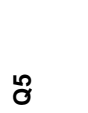 & \%ั & 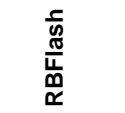 & 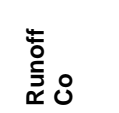 & 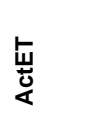 & $\begin{array}{l}\frac{3}{0} \\
\frac{d}{\omega}\end{array}$ & $\overline{\underline{\mathbf{u}}}$ & 읃 & 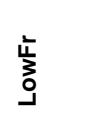 & 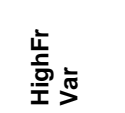 & 芌 & 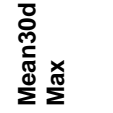 & $\begin{array}{l}\bar{n} \\
\overline{0} \\
\dot{0}\end{array}$ & 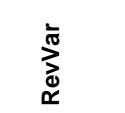 \\
\hline (Intercept) & -9.96 & 0.902 & 2.44 & 82 & $\begin{array}{ll}0.174 \\
\end{array}$ & 0.38 & 110 & -3.74 & 0.238 & -5.89 & 0.459 & 0.555 & 0.681 & -33.1 & & 0.214 \\
\hline area & & $-1.71 \mathrm{E}-06$ & & & $-3.61 \mathrm{E}-07$ & & & & & & & $1.64 \mathrm{E}-07$ & & & $-3.19 \mathrm{E}-08$ & $1.76 \mathrm{E}-08$ \\
\hline meanElev & & & & & & & & & & & & & & & & 4.06E-06 \\
\hline stdElev & 0.00574 & & & & & 0.000533 & & & & & & & & & & \\
\hline meanSlope & & & & 0.294 & 0.000488 & & -4.17 & & & & & & & & & \\
\hline Water & & -3.48 & & & -1.84 & & & -8.52 & 0.781 & -17.2 & & 1.07 & & & & \\
\hline \multirow{2}{*}{\multicolumn{17}{|c|}{\begin{tabular}{|l} 
Glacier \\
Urban
\end{tabular}}} \\
\hline & & & & & & & & & & & & & & & & \\
\hline \multirow{2}{*}{\multicolumn{17}{|c|}{\begin{tabular}{|l} 
Forest \\
Agriculture
\end{tabular}}} \\
\hline & & & & & & & & & & & & & & & & \\
\hline \multicolumn{17}{|l|}{ Pasture } \\
\hline \multicolumn{17}{|l|}{ Wetland } \\
\hline OpwithVeg & & 0.809 & 4.12 & & 0.151 & & & & -0.148 & & -0.245 & & & & 0.0503 & \\
\hline OpwithoutVeg & & & & & & & & & & -7.12 & & & & & 0.0412 & \\
\hline Irrigated & & 0.362 & -6.55 & 1.26 & & & & 1.97 & -0.00546 & & 5.17 & & & & & \\
\hline Coarse & 1.84 & -0.546 & 1.26 & & & 0.06 & -49.4 & 0.592 & 0.113 & & & & & & & \\
\hline \multirow{2}{*}{\multicolumn{17}{|c|}{$\begin{array}{l}\text { Medium } \\
\text { Fine }\end{array}$}} \\
\hline & & & & & & & & & & & & & & & & \\
\hline \multirow{2}{*}{\multicolumn{17}{|c|}{\begin{tabular}{|l} 
Organic \\
NoTexture
\end{tabular}}} \\
\hline & & & & & & & & & & & & & & & & \\
\hline \multicolumn{17}{|l|}{ Shallow } \\
\hline \multicolumn{17}{|l|}{ Moraine } \\
\hline \multicolumn{17}{|l|}{ DrainDens } \\
\hline Pmean & 0.0229 & & & & & & 0.249 & & & & & $-1.89 E-04$ & & & $-5.51 \mathrm{E}-05$ & $-8.67 \mathrm{E}-05$ \\
\hline Tmean & & 0.0397 & & & & & 25.4 & & & & & & & & & \\
\hline PSI & & & & & & & & 26.6 & & 51.1 & & & & 190 & & \\
\hline \multicolumn{17}{|c|}{-42.9} \\
\hline $\mathrm{Cz}$ & -3.46 & & & & & -0.218 & & & & & & & & & & \\
\hline \multicolumn{17}{|l|}{ CzMz } \\
\hline Mz & & -0.179 & & & & & & 1.33 & & 2.31 & & & & 9.07 & 0.00424 & \\
\hline \multirow{2}{*}{\multicolumn{17}{|c|}{ pCm }} \\
\hline & & & & & & & & & & & & & & & & \\
\hline \multicolumn{17}{|c|}{0.0265} \\
\hline Pzi & & & & 13.6 & & & & & & & & & & & & \\
\hline \multicolumn{17}{|l|}{ Pzm } \\
\hline PzpCmm & & & & & & & & & & & & & & & & \\
\hline
\end{tabular}




\begin{tabular}{|c|c|c|c|c|c|c|c|c|c|c|c|c|c|c|c|c|}
\hline & $\frac{0}{8}$ & गे & 40 & \%ั & 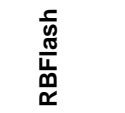 & 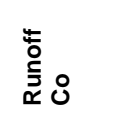 & 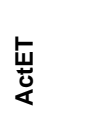 & $\begin{array}{l}\frac{3}{0} \\
\frac{d}{\omega}\end{array}$ & $\overline{\underline{\mathbf{u}}}$ & 을 & 崩 & $\begin{array}{l}\frac{\bar{L}}{5} \\
\frac{5}{5} \\
\frac{0}{I}\end{array}$ & 言 & 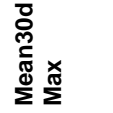 & $\begin{array}{l}\bar{n} \\
\overline{0} \\
0\end{array}$ & 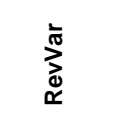 \\
\hline (Intercept) & -9.08 & 1.53 & -1.9 & $\begin{array}{ll}0.28 \\
\end{array}$ & 0.000435 & 0.719 & 287 & 2.68 & $\begin{array}{ll}0.318 \\
\end{array}$ & 5.6 & 0.482 & 0.358 & (0.832 & 5.41 & (0.108 & 0.239 \\
\hline \multirow{2}{*}{\multicolumn{17}{|c|}{$\begin{array}{l}\text { area } \\
\text { meanElev }\end{array}$}} \\
\hline & & & 0.00222 & & & 7.41E-05 & & & & & -0.00037 & & & & & \\
\hline \multirow{2}{*}{\multicolumn{17}{|c|}{$\begin{array}{l}\text { stdElev } \\
\text { meanSlope }\end{array}$}} \\
\hline & & & & & & & & & & & & & & & & \\
\hline \multirow{2}{*}{\multicolumn{17}{|c|}{ Water }} \\
\hline & & & & & & & & & & & & & & & & \\
\hline Urban & & -5.13 & & & & & & & & & -8.22 & & & & & -1.16 \\
\hline Forest & & -0.168 & & & & & & -1.49 & & -2.89 & & & & & & \\
\hline Agriculture & & & & & -0.0825 & -0.278 & & & & & & & & 12.7 & 0.0559 & \\
\hline Pasture & -4.09 & & & -6.11 & & & 129 & & & & & & & & & \\
\hline \multirow{2}{*}{\multicolumn{17}{|c|}{$\begin{array}{l}\text { Wetland } \\
\text { OpwithVeq }\end{array}$}} \\
\hline \multirow{2}{*}{\multicolumn{17}{|c|}{ OpwithoutVeg }} \\
\hline & & & & & & & & & & & & & & & & \\
\hline \multirow{2}{*}{\multicolumn{17}{|c|}{$\begin{array}{l}\text { Irrigated } \\
\text { Coarse }\end{array}$}} \\
\hline \multirow{2}{*}{\multicolumn{17}{|c|}{ Medium }} \\
\hline & & & & & & & & & & & & & & & & \\
\hline Fine & -7.51 & & & -20.2 & & -0.0734 & 237 & -1 & & & & & & -10.7 & -0.0142 & \\
\hline Organic & & -0.159 & 1.78 & & 0.215 & & & & -0.0343 & & & -0.0271 & & & & \\
\hline \multirow{2}{*}{\multicolumn{17}{|c|}{$\begin{array}{l}\text { NoTexture } \\
\text { Shallow }\end{array}$}} \\
\hline & & & & & & & & & & & & & & & & \\
\hline \multicolumn{17}{|l|}{ Moraine } \\
\hline DrainDens & & & & -177 & -0.305 & & & & & & 0.719 & & & & & \\
\hline Pmean & 0.0265 & -0.000278 & 0.00405 & 0.076 & 9.35E-05 & & 0.165 & & $-5.27 \mathrm{E}-05$ & & & & -0.000162 & & -3.44E-05 & $-9.07 E-05$ \\
\hline Tmean & & & & & 0.0154 & & & & & & & & & & & \\
\hline PSI & & & & & & & & & & & & 0.137 & & & & \\
\hline Al & & & & & 0.0196 & -0.188 & & & & & & & & & & \\
\hline \multirow{2}{*}{\multicolumn{17}{|c|}{$\begin{array}{l}\mathrm{Cz} \\
\mathrm{Cz} M \mathrm{Mz}\end{array}$}} \\
\hline & & & & & & & & & & & & & & & & \\
\hline Mz & & & & & & & & & & & & & & & & -0.0225 \\
\hline \multirow{2}{*}{\multicolumn{17}{|c|}{ pCm }} \\
\hline & & & & & & & & & & & & & & & & \\
\hline \multicolumn{17}{|c|}{-0.0911} \\
\hline \multirow{2}{*}{\multicolumn{17}{|c|}{$\begin{array}{l}\text { Pzi } \\
\text { Pzm }\end{array}$}} \\
\hline & & & & & & & & & & & & & & & & \\
\hline PzpCmm & & & & & & & & & & & & & & & & \\
\hline
\end{tabular}




\begin{tabular}{|c|c|c|c|c|c|c|c|c|c|c|c|c|c|c|c|c|}
\hline & $\frac{0}{8}$ & ơ & 용 & ஜூ & 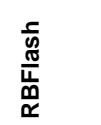 & 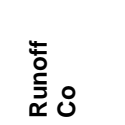 & 岀 & $\begin{array}{l}\frac{3}{0} \\
\frac{\mathbf{d}}{\omega}\end{array}$ & $\stackrel{\bar{u}}{\boldsymbol{~}}$ & 只 & 梈 & 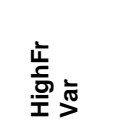 & 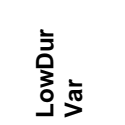 & 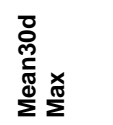 & $\begin{array}{l}\overline{\mathbf{n}} \\
\overline{0}\end{array}$ & 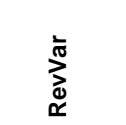 \\
\hline (Intercept) & -6.81 & 1.28 & 4.34 & -3.63 & 0.115 & 0.65 & 373 & 2.63 & 0.232 & 5.74 & \begin{tabular}{l|l}
0.336 \\
\end{tabular} & 0.538 & 0.7 & -25.6 & 0.0937 & 0.175 \\
\hline area & $-2.91 \mathrm{E}-06$ & & & $-1.36 \mathrm{E}-05$ & & $-9.44 \mathrm{E}-08$ & $5.49 \mathrm{E}-05$ & & $5.29 \mathrm{E}-07$ & & & $1.71 \mathrm{E}-07$ & & & & \\
\hline meanElev & & & -0.000165 & & & & & & 3.03E-05 & & & & & & & $-2.66 \mathrm{E}-06$ \\
\hline stdElev & & & $\begin{array}{r}0.00938 \\
\end{array}$ & -0.037 & & & & & & -0.00303 & & & -0.000175 & -0.0082 & & $-8.56 \mathrm{E}-05$ \\
\hline meanSlope & & & & 0.947 & & 0.00648 & -0.825 & & & & & & & & & \\
\hline Water & & -4.96 & 11.9 & 12.8 & -1.55 & & & -14.4 & 0.597 & -25.9 & & 1.06 & & 119 & & 0.829 \\
\hline Glacier & & & 13.3 & & & & & & & & & & & & & \\
\hline Urban & -65.3 & & & & & & & & & & -8.72 & & & & & \\
\hline \multicolumn{17}{|l|}{ Forest } \\
\hline Agriculture & & & & & -0.0161 & & & & & & & & & & & \\
\hline Pasture & & & & 8.64 & 0.205 & -0.0586 & 2.68 & -2.27 & & -3.54 & & & & & & \\
\hline \multicolumn{17}{|l|}{ Wetland } \\
\hline OpwithVeg & 17.1 & & & & 0.227 & & -457 & & & & & & & 48.8 & & \\
\hline OpwithoutVeg & & & & & & & & & -0.000312 & & & & & & & \\
\hline Irrigated & & & & & & & & & 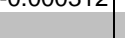 & 17.2 & 5.51 & & & & & \\
\hline Coarse & & -0.475 & 1.18 & & & 0.0396 & & -1.28 & & -3.88 & & & & -11.1 & & \\
\hline \multirow{2}{*}{\multicolumn{17}{|c|}{$\begin{array}{l}\text { Medium } \\
\text { Fine }\end{array}$}} \\
\hline & & & & & & & & & & & & & & & & \\
\hline \multicolumn{17}{|l|}{ Organic } \\
\hline NoTexture & & & & & & & & & & & & & & & -0.117 & \\
\hline \multirow{2}{*}{\multicolumn{17}{|c|}{\begin{tabular}{|l} 
Shallow \\
Moraine
\end{tabular}}} \\
\hline & & & & & & & & & & & & & & & & \\
\hline \multicolumn{17}{|l|}{ DrainDens } \\
\hline Pmean & 0.0265 & & & 0.0724 & & & & & & & & $-1.87 \mathrm{E}-04$ & & & & \\
\hline Tmean & -0.68 & & & -2.87 & & & 23 & & -0.00118 & & & & & 3.29 & & -0.00342 \\
\hline PSI & & & -0.867 & & & 0.362 & & & & & & 0.0726 & 0.0166 & & & \\
\hline Al & & & -2.61 & & 0.0259 & -0.299 & & & & & & & & & & \\
\hline $\mathrm{Cz}$ & & & 0.964 & & & & & & & & & & & & & \\
\hline \multirow{2}{*}{\multicolumn{17}{|c|}{$\begin{array}{l}\mathrm{CzMz} \\
\mathrm{Mz}\end{array}$}} \\
\hline & \multirow{2}{*}{\multicolumn{16}{|c|}{$\mathrm{Mz}$}} \\
\hline $\mathrm{pCm}$ & & & & & & 0.115 & -80.9 & & & & & & & & & \\
\hline pCmi & & & & & -0.0128 & & & & & & & & & & & \\
\hline \multirow{2}{*}{\multicolumn{17}{|c|}{ Pzi }} \\
\hline & & & & & & & & & & & & & & & & \\
\hline Pzm & & & -2.09 & & & & & & -0.143 & & 0.0491 & & & & & \\
\hline PzpCmm & & & & 12.1 & & 0.0489 & & & -0.0812 & -0.0416 & & & & -2.76 & & \\
\hline
\end{tabular}

